NBER WORKING PAPER SERIES

\title{
ROBUSTLY OPTIMAL MONETARY POLICY IN A NEW KEYNESIAN MODEL WITH HOUSING
}

\author{
Klaus Adam \\ Michael Woodford
}

Working Paper 26833

http://www.nber.org/papers/w26833

\author{
NATIONAL BUREAU OF ECONOMIC RESEARCH \\ 1050 Massachusetts Avenue \\ Cambridge, MA 02138 \\ March 2020
}

We thank Pierpaolo Benigno, Massimo Marinacci, Jianjun Miao, Lars Svensson, Luigi Paciello and Monika Piazzesi for helpful comments. This paper supersedes our earlier drafts titled "Leaning Against Housing Prices as Robustly Optimal Monetary Policy" and "Housing Prices and Robustly Optimal Monetary Policy." The research of Klaus Adam has been supported by CRC-TR224 (Project C2) of the German Research Foundation (DFG). Any remaining errors are our own. The views expressed herein are those of the authors and do not necessarily reflect the views of the National Bureau of Economic Research.

NBER working papers are circulated for discussion and comment purposes. They have not been peer-reviewed or been subject to the review by the NBER Board of Directors that accompanies official NBER publications.

(C) 2020 by Klaus Adam and Michael Woodford. All rights reserved. Short sections of text, not to exceed two paragraphs, may be quoted without explicit permission provided that full credit, including $(\odot$ notice, is given to the source. 
Robustly Optimal Monetary Policy in a New Keynesian Model with Housing Klaus Adam and Michael Woodford

NBER Working Paper No. 26833

March 2020

JEL No. C61,E52

\begin{abstract}
$\underline{\text { ABSTRACT }}$
We analytically characterize optimal monetary policy for an augmented New Keynesian model with a housing sector. With rational private sector expectations about housing prices and inflation, optimal monetary policy can be characterized by a standard "target criterion" in terms of inflation and the output gap, that makes no reference to housing prices. If instead the policymaker is concerned with potential departures of private sector expectations from rational ones, and seeks a policy that is robust against such possible departures, then the optimal target criterion will also depend on housing prices. For empirically realistic cases, robustness requires the central bank to "lean against" housing prices, i.e., to adopt a stance that is projected to undershoot (overshoot) its normal targets for inflation and the output gap following unexpected housing price increases (decreases). Notably, robustly optimal policy does not require that the central bank distinguish between "fundamental" and "non-fundamental" movements in housing prices.
\end{abstract}

Klaus Adam

University of Oxford

Nuffield College

New Road

Oxford OX1 1NF

United Kingdom

klaus.adam@economics.ox.ac.uk

Michael Woodford

Department of Economics

Columbia University

420 W. 118th Street

New York, NY 10027

and NBER

mw2230@columbia.edu 


\section{Introduction}

The question of how (if at all) asset price movements should be taken into account in a central bank's interest-rate policy decisions has been much debated since at least the 1990s. ${ }^{1}$ The importance of the issue has become even more evident after the fallout for the global economy of the recent real estate booms and busts in the US and several other countries, which at least some attribute to monetary policy decisions that failed to take account of the consequences for the housing market. ${ }^{2}$

Yet the issue is not easily addressed using standard frameworks for monetary policy analysis. One reason is that it is often supposed that large movements in asset prices are particularly problematic when they are not justified by economic "fundamentals," but instead represent mistaken valuations resulting from mistaken expectations. ${ }^{3}$ An analysis that evaluates alternative monetary policies under the assumption that the outcome resulting from each candidate policy will be a rational-expectations equilibrium assumes that there can never be any misallocation of resources due to speculative mispricing of assets, regardless of the monetary policy that is chosen. Such an analysis will accordingly conclude that there is no need for a central bank to monitor or respond to signs of such mispricing — but by assuming away the problem.

Some analyses of the question have accordingly allowed for potential departures of asset prices from "fundamental" values, introducing an expectational error term in the asset pricing equation that is specified as an exogenous stochastic process (e.g., Bernanke and Gertler (1999, 2001), Galí (2014)). But conclusions from such analyses depend on what is assumed about the nature of expectational errors, as illustrated in Miao, Shen and Wang (2020). In addition, it depends on how the probability distribution of errors under some given policy (perhaps the kind of policy that has historically been implemented) is assumed to change with each alternative policy that may be contemplated. Yet there is little basis for confidence about the correctness of a particular choice in this regard.

In recent work, Caines and Winkler (2019) consider a model where asset price beliefs are allowed to deviate from model consistent ones, following the approach in Adam, Marcet and Nicolini (2016). They show that it is optimal for monetary policy to lean against asset prices, when assuming that the process governing belief formation in the asset market is invariant to policy.

Here we propose a different approach to the problem. It neither assumes that expectations must necessarily be model-consistent, nor that expectational errors must be of any specific type that can be predicted in advance; rather, it associates with any contemplated policy a set of possible probability beliefs, that includes all possible (internally coherent) probability beliefs that are not too different from those predicted by one's model, in the case of that policy and those beliefs. This is the hypothesis of "near-rational expectations" (NRE) introduced in Woodford (2010).

This makes the set of possible private-sector beliefs contemplated by the policy analysis dependent on the particular policy that is adopted, as in the case of the rational expectations hypothesis. In particular, beliefs are treated as possible if it would not be too easy to disconfirm them using observed data from the equilibrium of the model, and whether this is so will depend on policy. But the set of beliefs that are considered will include ones that result in asset valuations different from the ones that will be judged correct according to the policy analyst's model; hence the policy analyst will consider the possibility of equilibria in which assets are mispriced, and will therefore consider the consequences of responding to such asset price movements

\footnotetext{
${ }^{1}$ See, for example, Bernanke and Gertler (1999, 2001), Gilchrist and Leahy (2002), Christiano et al. (2010)

${ }^{2}$ For example, Taylor (2007) or Adam, Marcet and Kuang (2012).

${ }^{3}$ Adam, Marcet and Beutel (2017) show that stock market investors' expectations are mistaken, in the sense that they display periods of over-optimism and over-pessimism.
} 
in different ways.

Because the set of possible "near-rational" beliefs associated with any given policy includes many elements, analysis of the kind proposed here will not associate a single predicted path for the economy (contingent on the realized values of exogenous shocks) with a given policy. It may therefore be wondered how welfare comparisons of alternative policies are possible. Our proposal, in the spirit of the robust policy analysis of Hansen and Sargent (2008), is to choose a policy that achieves the highest possible lower bound for expected utility of the representative household, across all of the equilibria with "near-rational" expectations consistent with that policy. ${ }^{4}$ We call a solution to this problem a "robustly optimal" policy rule.

We wish to consider the problem of robustly optimal policy within as broad a class of possible policy rules as possible; in particular, we do not wish to prejudge questions such as the way in which the policy rule may involve systematic response to housing-related variables or to indicators of market expectations. Our earlier paper without a housing sector (Adam and Woodford (2012)) shows how it is possible to characterize robustly optimal policy rules without restricting oneself a priori to some simple parametric family of policy rules. The basic idea is that one can in a first step derive an upper bound for the maximin level of welfare that is potentially achievable under any policy rule. This upper-bound welfare level can be determined without making reference to any specific class of policy rules. In a second step, one can then display examples of policy rules that achieve this upper-bound outcome.

Here we generalize our earlier approach in a number of important ways. First, we extend the New Keynesian DSGE model used in our earlier analysis to include an endogenous housing supply, equilibrium fluctuations in housing prices, and potentially distorted housing price expectations. Second, we make a methodological advance by presenting a new Lagrangian characterization of the upper-bound dynamics, the so-called Lagrangian Upper-Bound Problem (LUBP). The LUBP makes it considerably easier to verify that given dynamics represent an upper-bound on what robustly optimal policy can achieve (as maximized worst-case outcome).

Finally, we present a novel method for parameterizing our non-parametric set of admitted belief distortions. This method turns out to be analytically convenient and allows handling larger belief distortions, which affect the equilibrium dynamics of inflation and housing prices to first order. The policies considered in the present paper are thus robust to belief deviations in a stronger sense than in Adam and Woodford (2012), where belief distortions were restricted to affect equilibrium dynamics (for a given policy) to second order only. Despite these generalizations, we can still derive analytical expressions characterizing optimal monetary policy.

We are especially interested in a particular way of specifying the policy rule, in which the central bank commits itself to fulfill a quantitative target criterion at all times. ${ }^{5}$ Under this commitment it uses its policy instrument at each point in time as necessary in order to ensure that the paths of various endogenous variables satisfy the relationship specified by the target criterion. In a basic New Keynesian model without a housing sector and under the assumption

\footnotetext{
${ }^{4}$ Hansen and Sargent assume a policy analyst who is herself uncertain that her model is precisely correct as a description of the economy; when the expectations of other economic agents are an issue in the analysis, these are typically assumed to share the policy analyst's model, and her concerns about mis-specification and preference for robustness as well. We are instead concerned about potential discrepancies between the views of the policy analyst and those of the public; and the potential departures from model-consistent beliefs on the part of the public are not assumed to reflect a concern for robustness on their part. In Benigno and Paciello (2014), instead, optimal policy is computed under the assumption that members of the public are concerned about the robustness of their own decisions, and the policymaker correctly understands the way that this distorts their actions (relative to what the policymaker believes would be optimal for them).

${ }^{5}$ The robustly optimal policy rule is not unique, as is discussed in more detail in Adam and Woodford (2012). Different rules may be consistent with the same worst-case NRE equilibrium dynamics, and so achieve the same lower bound for expected utility, without being equivalent, either in terms of the out-of-equilibrium behavior that they would require from the central bank, or in terms of the boundaries of the complete set of NRE equilibria consistent with the policy in question.
} 
of rational expectations, the optimal policy commitment can be characterized in these terms, see Benigno and Woodford (2005), Giannoni and Woodford (2017); the required target criterion is a "flexible inflation targeting" rule in the sense of Svensson (1999), in which short-run departures from the long-run inflation target are justified precisely to the extent that they are proportional to short-run variations in the rate of change of an "output gap" variable.

We first show that if the policymaker assumes private agents to have rational expectations, commitment to a target criterion involving inflation $\left(\pi_{t}\right)$ and an appropriately defined output gap $\left(y_{t}^{g a p}\right)$ is sufficient for implementing optimal monetary policy, even in the model with distortions in the housing sector. The target criterion can be written in the form

$$
\pi_{t}+\lambda_{y}\left(y_{t}^{g a p}-y_{t-1}^{g a p}\right)=0
$$

where $\lambda_{y}>0$. While shocks to housing supply and housing demand do affect the definition of the target output level and hence the definition of the output gap $y_{t}^{g a p}$, the target criterion makes no reference to housing prices.

We then show that this ceases to be the case when the policymaker fears possible deviations of housing price and inflation expectations from the model-consistent ones; yet a generalization of the standard target criterion can still be used to implement robustly optimal policy. This target criterion involves inflation and the rate of change of the output gap, with identical coefficients as in the case with rational expectations, but now must additionally involve the surprises to housing prices $\left(\widehat{q}_{t}^{u}\right)$ and inflation:

$$
\pi_{t}+\lambda_{y}\left(y_{t}^{g a p}-y_{t-1}^{g a p}\right)+\lambda_{\pi}\left(\pi_{t}-E_{t-1} \pi_{t}\right)+\lambda_{q}\left(\widehat{q}_{t}^{u}-E_{t-1} \widehat{q}_{t}^{u}\right)=0,
$$

where $\lambda_{\pi}>0$ and $\lambda_{q} \gtrless 0$.

In the empirically realistic case in which housing is subsidized by the government, and is therefore over-supplied in equilibrium, and in which output falls short of its optimum, because of monopoly power and tax distortions, we have $\lambda_{q}>0$. The robustly optimal target criterion then requires the central bank to "lean against" unexpected increases in housing prices. By this we mean that it should adopt a policy stance that is projected to result in smaller increases in inflation and/or the output gap than would be chosen under the assumption of rational housing price and inflation expectations. A positive housing price surprise thus requires a "tighter" monetary policy than suggested by a rational-expectations analysis. Similarly, the policymaker should aim for larger increases in inflation and or the output gap and thus "looser" policy in the case of an unexpected decline in housing prices.

Notably, the robustly optimal targeting rule makes no reference to the "fundamental" housing price. Instead, it only involves responses to housing price surprises, independently of whether these surprises reflect changes in fundamentals or changes in the size of belief distortions. This makes the target criterion more suitable as a basis for communication with the public about the way that policy decisions are to be justified, as it does not require the central bank to take a public position with regard to the degree to which housing prices differ from their fundamental values. The difficulty of determining fundamental asset values in real time is often used as an excuse for refraining from any attempt to "lean against" asset bubbles; but in our proposal, "leaning against" housing price increases does not require any such determination.

The policy of "leaning against" house price increases is more robust than a correspondingly flexible inflation targeting rule that ignores house price and inflation surprises, in the sense that the distorted expectations that would lead to the worst possible outcome under this policy do not lower welfare as much as some possible beliefs distortions would under the conventional policy. ${ }^{6}$ In particular, in a setting with an excess supply of housing and a suboptimally low

\footnotetext{
${ }^{6}$ This assumes that belief distortions must comply in both cases with the same maximum bound on the possible size of belief distortions.
} 
output level, the policymaker is most fearful of belief distortions that simultaneously increase housing price expectations and inflation expectations. Increased housing price expectations cause current housing prices to increase and thereby lead to an even larger supply of housing in a setting where there are already too many houses. Similarly, higher inflation expectations imply - via the New Keynesian Phillips curve - an even lower output level for any given level of inflation, in a situation where output is already suboptimally low. By engineering a (more) negative correlation between housing price and inflation surprises - as implied by policies that "lean against" housing prices - belief distortions are less able to simultaneously increase both of these expectations. This helps to reduce the maximum harm that belief distortions of any given size can possibly inflict. The degree to which the robustly optimal policy requires "leaning against" house prices increases depends, however, on model parameters. Notably, it depends both on the size of the housing subsidy and on the size of the output shortfall, as discussed further below.

The paper is structured as follows. Section 2 defines robustly optimal policy and presents the general approach that we use to characterize it. Section 3 presents our New Keynesian monetary DSGE model with a housing sector, and defines an equilibrium with possibly distorted private sector expectations, generalizing the standard concept of rational-expectations equilibrium. Section 4 considers optimal monetary policy under the assumption of rational expectations. It demonstrates the irrelevance of housing prices for monetary policy by deriving a targeting rule that implements optimal monetary policy and that depends on inflation and the output gap only. Section 5 considers equilibrium dynamics with distorted expectations. It explains in what sense we allow for larger belief distortions than in our earlier work and how these give rise to first-order terms in the linear approximations to the model structural equations. It also presents the kind of linear dynamics that we admit as possible solutions. Section 6 determines the upper bound on what monetary policy can maximally achieve in the presence of belief distortions. It presents the nonlinear first-order conditions, as well as the linearized equations characterizing the optimal linear upper-bound dynamics. We also show how to verify secondorder conditions so as to insure that the presented dynamics in fact represent an optimum from the policymaker's perspective. Section 7 then presents the robustly optimal targeting criterion. It discusses under what conditions it involves "leaning against" housing prices and how one can (numerically) verify that it attains the upper-bound solution as worst-case outcome. We compare the outcomes associated with robustly optimal and rational expectations optimal policies in a numerical illustration in section 8. A conclusion briefly summarizes. Technical material is relegated to a series of appendices.

\section{The Policy Problem in General Terms}

This section describes the general approach that we use to characterize robustly optimal policy. Section 2.1 defines the problem and presents our new Lagrangian formulation of the upperbound problem. Subsection 2.2 introduces non-parametric forms of distorted private sector beliefs and an entropy-based measure for capturing the size of belief-distortions, following earlier work in Woodford (2010) and Adam and Woodford (2012). Section 2.3 provides a novel approach that allows us to parameterize the admitted set of belief distortions. This approach will be analytically convenient when applying the general ideas spelled out in this section to a New Keynesian model with a housing sector in the remaining part of the paper, and allows for larger belief distortions than the methods used in our earlier work. 


\subsection{Robustly Optimal Policy}

Consider a policymaker who cares about some vector $y$ of endogenous economic outcomes in the sense of seeking to achieve as high a value as possible for some (welfare) objective $W(y)$. The value of $y$ depends both on policy and on forward-looking private sector decisions, which in turn depend on the private-sector's belief distortions as parameterized by some vector $\zeta$. Among the determinants of $y$ is a set of structural economic equations, typically involving first-order conditions of private agents and market clearing conditions, that we write as

$$
F(y, \zeta)=0
$$

We assume that the equations (2) are insufficient to completely determine the vector $y$, under given belief distortions $\zeta$, so that the policymaker faces a non-trivial choice.

Let us suppose that the policymaker must choose a policy commitment $c$ from some set $C$ of feasible policy commitments. Our results about robustly optimal policy do not depend on the precise specification of the set $C$; for now, we simply assume that there exists such a set, but we make no specific assumption about what its boundaries may be. We only impose two general assumptions about the nature of the set $C$ : first, we assume that each of the commitments in the set $C$ can be defined independently of what the belief distortions may be ${ }^{7}$; and second, we shall require that for any $c \in C$, there exists an equilibrium outcome for any choice of $\zeta$ in some set of possible belief distortions $\mathcal{Z}$. This last assumption assigns to the policymaker the responsibility for insuring existence of equilibrium for arbitrary belief distortions.

Given our general requirements, the set $C$ may include many different types of policy commitments. For example, it may involve policy commitments that depend on the history of exogenous shocks; commitments that depend on the history of endogenous variables, as is the case with Taylor rules; and commitments regarding relationships between endogenous variables, as is the case with so-called targeting rules. Also, the endogenous variables in terms of which the policy commitment is expressed may include asset prices (futures prices, forward prices, etc.) that are often treated by central banks as indicators of private-sector expectations, as long as the requirement is satisfied that the policy commitment must be consistent with belief distortions of an arbitrary form.

In order to define the robustly optimal decision problem of the policymaker, we further specify that the equilibrium outcome $y$ associated with a given policy commitment $c \in C$ and a given belief distortion $\zeta$ are given by an outcome function

$$
O: \mathcal{Z} \times C \rightarrow Y
$$

with the property that

$$
F(O(\zeta, c), \zeta)=0
$$

for all all $\zeta \in \mathcal{Z}$ and $c \in C$. Here we have not been specific about what we mean by an "equilibrium," apart from the fact that (2) must be satisfied. In the context of the specific model presented in the next section, equilibrium has a precise meaning. For purposes of the present discussion, it does not actually matter how we define equilibrium; only the definition of the outcome function matters for our subsequent discussion. ${ }^{8}$

\footnotetext{
${ }^{7}$ As is made more specific in the application below, we specify policy commitments by equations involving the endogenous and exogenous variables, but not explicitly the belief distortions. Of course, the endogenous variables referred to in the policy commitment will typically also be linked by structural equations that involve the belief distortions.

${ }^{8}$ If the set of equations (2) is not a complete set of requirements for $y$ to be an equilibrium, this only has the consequence that the upper-bound outcome defined below might not be a tight enough upper bound; it does not affect the validity of the assertion that it provides an upper bound.
} 
Our definition of robustly optimal policy depends on a function $V(\zeta, y) \geq 0$ that measures the size of the belief distortions. We assume that $V(\zeta, y)$ is equal to zero only in the case of beliefs that agree precisely with those of the policymaker, and that higher values of $V(\zeta, y)$ represent more severe distortions. The functional form for $V(\zeta, y)$ ultimately reflects our conception of "near-rational expectations." Section 2.2 introduces a specific functional form, based on a relative entropy measure, but our remarks here would also apply to other measures of belief distortions.

The robustly optimal policy problem can then be represented as a choice of a policy commitment that solves

$$
\max _{c \in C}\left\{\min _{\zeta \in \mathcal{Z}} W(O(\zeta, c)) \text { s.t. } V(\zeta, O(\zeta, c)) \leq \bar{V}\right\}
$$

where $\bar{V} \geq 0$ measures the policymaker's degree of concern for robustness. For the special case with $\bar{V}=0$ the robustly optimal policy problem reduces to a standard optimal policy problem with model-consistent private sector expectations. As $\bar{V}$ increases, the policymaker becomes concerned with increasingly larger deviations of private sector expectations from those that would be consistent with its own model used for policy analysis.

Let $c^{R}$ denote the robustly optimal policy commitment and $\zeta^{R}$ the associated worst-case beliefs, i.e., the solution to the inner problem in (3). Suppose that there exists a Lagrange multiplier $\theta \geq 0$ such that $\zeta^{R}$ also solves

$$
\min _{\zeta \in \mathcal{Z}} W\left(O\left(\zeta, c^{R}\right)\right)+\theta V\left(\zeta, O\left(\zeta, c^{R}\right)\right)
$$

with $\theta\left[V\left(\zeta, O\left(\zeta, c^{R}\right)\right)-\bar{V}\right]=0$. Then $c^{R}$ and $\zeta^{R}$ also jointly solve the alternative problem

$$
\max _{c \in C} \min _{\zeta \in \mathcal{Z}} U(O(\zeta, c), \zeta)
$$

where

$$
U(y, \zeta) \equiv W(y)+\theta V(\zeta, y)
$$

and the parameter $\theta$ used in the definition of $U(y, \zeta)$ parameterizes the concern for robustness. In what follows, we shall define the robustly optimal policy problem in this way, taking the value of $\theta$ as part of the definition of our problem. The formulation of the policy problem in equation (4) is of the same form as decision problem (3) discussed in Hansen and Marinacci (2016) and can alternatively be microfounded through a variational preference approach (Maccheroni, Marinacci and Rustichini (2006)).

Our general strategy for characterizing robustly optimal policy can be summarized as follows. Let the worst-case beliefs associated with a policy commitment be defined by the problem

$$
K(c) \equiv \min _{\zeta \in \mathcal{Z}} U(O(\zeta, c), \zeta)
$$

Let us also consider the maximization problem

$$
J(\zeta) \equiv \max _{y \in Y} U(y, \zeta) \quad \text { s.t. } F(y, \zeta)=0 .
$$

We then observe that

$$
\max _{c \in C} K(c) \leq \min _{\zeta \in \mathcal{Z}} \max _{c \in C} U(O(\zeta, c), \zeta) \leq \min _{\zeta \in \mathcal{Z}} J(\zeta)
$$

The optimization problem on the r.h.s. of inequality (7) provides an upper bound to the robustly optimal policy problem, i.e., the problem on the l.h.s. of inequality (7). In the upper-bound 
problem, belief distortions $\zeta$ are chosen first and the remaining outcomes $y$ second. In the robustly optimal policy problem, the policymaker must first make a policy commitment $c$ and the belief distortions $\zeta$ to be chosen second.

We can obtain a potentially weaker upper bound, by considering the Lagrangian UpperBound Problem (LUBP)

$$
\min _{\zeta \in \mathcal{Z}} \max _{y \in Y} U(y, \zeta)-\varphi^{\prime} F(y, \zeta)
$$

for some vector of Lagrange multipliers $\bar{\varphi}$. Suppose that there exists a vector of multipliers $\bar{\varphi}$ such that the solution $(\bar{\zeta}, \bar{y})$ to problem $(8)$ satisfies $F(\bar{y}, \bar{\zeta})=0$. Then one can show that $\bar{y}$ also solves the maximization problem $(6)$ when $\zeta=\bar{\zeta}$.

Furthermore, if we let $\bar{J}(\zeta ; \varphi)$ be the maximized value of the inner problem in the LUBP (8), we observe that

$$
\begin{aligned}
J(\zeta) & =\max _{y \in Y} \min _{\varphi} U(y, \zeta)-\varphi^{\prime} F(y, \zeta) \\
& \leq \max _{y \in Y} U(y, \zeta)-\bar{\varphi}^{\prime} F(y, \zeta)=\bar{J}(\zeta ; \bar{\varphi})
\end{aligned}
$$

It follows that the solution to the LUBP (8) when $\varphi=\bar{\varphi}$ provides an upper bound for the solution to the problem on the right-hand side of (7), and hence that

$$
\max _{c \in C} K(c) \leq \min _{\zeta \in \mathcal{Z}} J(\zeta) \leq \min _{\zeta \in \mathcal{Z}} \bar{J}(\zeta ; \bar{\varphi})
$$

Thus the solution to the LUBP (8) when $\varphi=\bar{\varphi}$ provides another upper bound for the robustly optimal policy problem. This would be true for any vector of multipliers $\varphi$, but when $\varphi=\bar{\varphi}$, this upper bound is attained by the solution to the problem in the middle of (9). Hence this is the upper bound of interest to us.

The previous observations provide a new strategy for determining robustly optimal monetary policy. We will first find Lagrange multipliers $\bar{\varphi}$ for which the solution to the LUBP (8) satisfies the structural equations $F(\bar{y}, \bar{\zeta})=0$, and characterize the outcome and belief distortions that solve that problem. We then demonstrate that this upper bound is achievable by some policy commitment $\bar{c}$. If we can do this, $\bar{c}$ must then be at least one example of a robustly optimal policy commitment. This approach has the advantage of allowing us to study the LUBP, which can be defined independently of any particular class of policy rules $C$, and then simply demonstrate that some particular rule $\bar{c}$ satisfies the requirements for robust optimality, without ever having to define the complete set of possible policy rules, let alone determine the worst-case beliefs for all of them.

More precisely, we proceed as follows. We begin by considering the first-order conditions (FOCs) for the problem in the middle of $(9)$, and use these to find a pair $(\bar{\zeta}, \bar{y})$ that represent a candidate solution to this problem. Because $\bar{y}$ satisfies the FOCs for problem (6), there must exist a vector of Lagrange multipliers $\bar{\varphi}$ such that $\bar{y}$ also satisfies the FOCs for the inner problem in the LUBP (8). We then use those same FOCs to construct a policy rule $\bar{c}$ that is consistent with outcome $\bar{y}$ in the case of belief distortions $\bar{\zeta}$.

It is then only necessary to verify (i) that $\bar{y}$ not only satisfies the FOCs for the inner problem of in the LUBP (8), but is an actual maximum, and more generally that $(\bar{\zeta}, \bar{y})$ solve the LUBP (8) when $\varphi=\bar{\varphi}$; (ii) that $\bar{y}$ is not only consistent with policy $\bar{c}$, but is the unique outcome determined by policy $\bar{c}$ in the case of beliefs $\bar{\zeta}$; and (iii) that $\bar{\zeta}$ are the worst-case belief distortions in the case of policy $\bar{c}$. If these additional three conditions are verified, we will have established that $\bar{c}$ is an example of a robustly optimal policy commitment. 


\subsection{Distorted Private Sector Expectations}

We next discuss our quantitative measure of belief distortions. At this point it becomes necessary to specify that our analysis concerns dynamic models in which information is progressively revealed over time, at a countably infinite sequence of successive decision points.

Let $(\Omega, \mathcal{B}, \mathcal{P})$ denote a standard probability space with $\Omega$ denoting the set of possible realizations of an exogenous stochastic disturbance process $\left\{\xi_{0}, \xi_{1}, \xi_{2}, \ldots\right\}, \mathcal{B}$ the $\sigma$-algebra of Borel subsets of $\Omega$, and $\mathcal{P}$ a probability measure assigning probabilities to any set $B \in \mathcal{B}$. We consider a situation in which the policy analyst assigns probabilities to events using the probability measure $\mathcal{P}$ but fears that the private sector may make decisions on the basis of a potentially different probability measure denoted by $\widehat{\mathcal{P}}$.

We let $E$ denote the policy analyst's expectations induced by $\mathcal{P}$ and $\widehat{E}$ the corresponding private sector expectations associated with $\widehat{\mathcal{P}}$. A first restriction on the class of possible distorted measures that the policy analyst is assumed to consider — part of what we mean by the restriction to "near-rational expectations" - is the assumption that the distorted measure $\widehat{\mathcal{P}}$, when restricted to events over any finite horizon, is absolutely continuous with respect to the correspondingly restricted version of the policy analyst's measure $\mathcal{P}$.

The Radon-Nikodym theorem then allows us to express the distorted private sector expectations of some $t+j$ measurable random variable $x_{t+j}$ as

$$
\widehat{E}\left[x_{t+j} \mid \xi^{t}\right]=E\left[\frac{\mathcal{M}_{t+j}}{\mathcal{M}_{t}} x_{t+j} \mid \xi^{t}\right]
$$

for all $j \geq 0$ where $\xi^{t}$ denotes the partial history of exogenous disturbances up to period $t$. The random variable $\mathcal{M}_{t+j}$ is the Radon-Nikodym derivative, and completely summarizes belief distortions. ${ }^{9}$ The variable $\mathcal{M}_{t+j}$ is measurable with respect to the history of shocks $\xi^{t+j}$, non-negative and is a martingale, i.e., satisfies

$$
E\left[\mathcal{M}_{t+j} \mid \omega^{t}\right]=\mathcal{M}_{t}
$$

for all $j \geq 0$. Defining

$$
m_{t+1}=\frac{\mathcal{M}_{t+1}}{\mathcal{M}_{t}}
$$

one step ahead expectations based on the measure $\widehat{\mathcal{P}}$ can be expressed as

$$
\widehat{E}\left[x_{t+1} \mid \xi^{t}\right]=E\left[m_{t+1} x_{t+1} \mid \xi^{t}\right],
$$

where $m_{t+1}$ satisfies

$$
E\left[m_{t+1} \mid \xi^{t}\right]=1 \text { and } m_{t+1} \geq 0 .
$$

This representation of the distorted beliefs of the private sector is useful in defining a measure of the distance of the private-sector beliefs from those of the policy analyst. As discussed in Hansen and Sargent (2005), the relative entropy

$$
R_{t}=E_{t}\left[m_{t+1} \log m_{t+1}\right]
$$

is a measure of the distance of (one-period-ahead) private-sector beliefs from the policymaker's beliefs with a number of appealing properties.

\footnotetext{
${ }^{9}$ See Hansen and Sargent (2005) for further discussion.
} 
We wish to extend this measure of the size of belief distortions to an infinite-horizon economy with a stationary structure. In the kind of model with which we are concerned, the policy objective in the absence of a concern for robustness is of the form

$$
W(y) \equiv E_{0}\left[\sum_{t=0}^{\infty} \beta^{t} U\left(y_{t}\right)\right],
$$

for some discount factor $0<\beta<1$, where $U(\cdot)$ is a time-invariant function, and $y_{t}$ is a vector describing the real allocation of resources in period $t$. Correspondingly, we propose to measure the overall degree of distortion of private-sector beliefs by a discounted criterion of the form

$$
E_{0}\left[\sum_{t=0}^{\infty} \beta^{t+1} m_{t+1} \log m_{t+1}\right],
$$

as in Woodford (2010) and Adam and Woodford (2012). This is a discounted sum of the one-period-ahead distortion measures $\left\{R_{t}\right\}$. We assign relative weights to the one-period-ahead measures $R_{t}$ for different dates and different states of the world in this criterion that match those of the other part of the policy objective (12). Use of this cost function implies that the policymaker's degree of concern for robustness (relative to other stabilization objectives) remains constant over time, regardless of past history.

\subsection{Parameterization of Belief Distortions}

In stating and solving the robustly optimal policy problem, it will be convenient to work with a simpler, parametric representation of the possible belief distortions. This parametric formulation will allow us to analytically handle belief distortions that are sufficiently large such that they affect equilibrium variables to first order (given monetary policy).

In the dynamic models that we consider, the structural equations (2) are a vector of conditions of the form

$$
F\left(y_{t-1}, y_{t}, \widehat{E}_{t}\left[g\left(y_{t+1}\right)\right] ; \xi_{t}\right)=0
$$

for each period $t$, where we use the notation $\widehat{E}_{t}\left[x_{t+1}\right]=\widehat{E}\left[x_{t+1} \mid \xi^{t}\right]$ for subjective expectations, and $x=g(y)$ is some function of the complete state vector $y$.

Suppose that the penalty for large belief distortions is given by (13). Then, the worst case beliefs associated with a robustly optimal policy problem of the form (4) will solve a problem of the form

$$
\begin{aligned}
& \min _{m_{t+1} \geq 0} E_{t}\left[\theta m_{t+1} \log m_{t+1}\right] \\
& \text { s.t. : } E_{t}\left[m_{t+1} x_{t+1}\right]=x_{t}^{e} \\
& E_{t}\left[m_{t+1}\right]=1
\end{aligned}
$$

for some distorted expectations $x_{t}^{e}$, and some parameter $\theta>0 .{ }^{10}$ The solution to this problem is given by

$$
\log m_{t+1}=\theta^{-1} \zeta_{t}^{\prime} x_{t+1}-\log E_{t}\left[\exp \left(\theta^{-1} \zeta_{t}^{\prime} x_{t+1}\right)\right]
$$

\footnotetext{
${ }^{10}$ The parameter $\theta$ might seem unnecessary since minimization of $E[\theta m \log m]$ is equivalent to minimization of $E[m \log m]$. Below, however, we are interested in characterizing the worst case beliefs in a limiting case where the amplitude of exogenous disturbances, and hence the variation in $x_{t+1}$ is made arbitrarily small, but the value of $\theta$ is made correspondingly small, so that there continue to be non-trivial differences in $m_{t+1}$ across states, regardless of how small the amplitude of the disturbances.
} 
where $\zeta_{t}$ is a vector of Lagrange multipliers associated with constraint (14). It therefore suffices to consider possible belief distortions of the form described by equation (15) and we can parameterize belief distortions in any period $t$ by the vector $\zeta_{t}$. Note that as in the notation used in section 2.1, the assumption of rational expectations will correspond to $\zeta_{t}=0$ at all times.

In the case of distorted expectations of this parametric form, we can evaluate the relative entropy (11), obtaining ${ }^{11}$

$$
R_{t}=R\left(\theta^{-1} \zeta_{t},\left\{x_{t+1}\right\}\right)
$$

The cost function (13) for a dynamic problem can then be evaluated as

$$
V(\zeta, y ; \theta)=E_{0} \sum_{t=0}^{\infty} \beta^{t+1} R\left(\theta^{-1} \zeta_{t},\left\{g\left(y_{t+1}\right)\right\}\right) .
$$

The upper bound on what robustly optimal policy can achieve will then be defined by a problem of the form given on the right-hand side of equation (7), where $W(y)$ is given by equation (12) and $V(\zeta, y)$ by equation (16).

\section{A Sticky Price Model with a Housing Sector}

We shall begin by deriving the exact structural relations describing a New Keynesian model featuring a long-lived asset and potentially distorted private sector expectations. The existing stock of assets is assumed to generate a service flow that directly enters agents' utility. Assets depreciate over time but can be produced using a technology with decreasing returns to scale. For convenience we interpret the long-lived asset as housing, though other interpretations are possible.

The model is completely standard, except for the presence of the long-lived asset and the fact that the private sector holds potentially distorted expectations. The exposition here extends the framework of Adam and Woodford (2011), who write the exact structural relations for a simpler model without a housing sector.

\subsection{Model Structure}

The economy is made up of identical infinite-lived households, each of which seeks to maximize

$$
U \equiv \widehat{E}_{0} \sum_{t=0}^{\infty} \beta^{t}\left[\tilde{u}\left(C_{t} ; \xi_{t}\right)-\int_{0}^{1} \tilde{v}\left(H_{t}(j) ; \xi_{t}\right) d j+\tilde{\omega}\left(D_{t} ; \xi_{t}\right)\right]
$$

subject to a sequence of flow budget constraints ${ }^{12}$

$$
\begin{aligned}
& P_{t} C_{t}+B_{t}+\left(D_{t}+(1-\delta) D_{t-1}\right) q_{t} P_{t}+k_{t} P_{t} \\
& \leq\left(1+s^{d}\right) \tilde{d}\left(k_{t} ; \xi_{t}\right) q_{t} P_{t}+\int_{0}^{1} w_{t}(j) P_{t} H_{t}(j) d j+B_{t-1}\left(1+i_{t-1}\right)+\Sigma_{t}+T_{t},
\end{aligned}
$$

where $\widehat{E}_{0}$ is the common distorted expectations held by consumers conditional on the state of the world in period $t=0, C_{t}$ an aggregate consumption good which can be bought at nominal price $P_{t}, H_{t}(j)$ is the quantity supplied of labor of type $j$ and $w_{t}(j)$ the associated real wage, $D_{t}$ the stock of durable assets or houses, $\delta \in[0,1]$ the housing depreciation rate, $q_{t}$ the real price

\footnotetext{
${ }^{11}$ The notation $\left\{x_{t+1}\right\}$ used below refers to the time $t$ conditional distribution of the random variable $x_{t+1}$.

${ }^{12}$ We abstract from state-contingent assets in the household budget constraint because the representative agent assumption implies that in equilibrium there will be no trade in these assets.
} 
of houses, $k_{t}$ investment in new houses and $\tilde{d}\left(k_{t} ; \xi_{t}\right)$ the resulting production of new houses, $s^{d} \gtrless 0$ a government subsidy or tax applied to the value of newly produced houses, $B_{t}$ nominal bond holdings, $i_{t}$ the nominal interest rate, and $\xi_{t}$ is a vector of exogenous disturbances, which may induce random shifts in the functions $\tilde{u}, \tilde{v}, \tilde{\omega}$ and $\tilde{d}$. The variable $T_{t}$ denotes lump sum taxes levied by the government and $\Sigma_{t}$ profits accruing to households from the ownership of firms.

The aggregate consumption good is a Dixit-Stiglitz aggregate of consumption of each of a continuum of differentiated goods,

$$
C_{t} \equiv\left[\int_{0}^{1} c_{t}(i)^{\frac{\eta-1}{\eta}} d i\right]^{\frac{\eta}{\eta-1}},
$$

with an elasticity of substitution equal to $\eta>1$. We further assume isoelastic functional forms

$$
\begin{gathered}
\tilde{u}\left(C_{t} ; \xi_{t}\right) \equiv \frac{C_{t}^{1-\tilde{\sigma}^{-1}} \bar{C}_{t}^{\tilde{\sigma}^{-1}}}{1-\tilde{\sigma}^{-1}}, \\
\tilde{v}\left(H_{t} ; \xi_{t}\right) \equiv \frac{\lambda}{1+\nu} H_{t}^{1+\nu} \bar{H}_{t}^{-\nu}, \\
\tilde{\omega}\left(D_{t} ; \xi_{t}\right)=\xi_{t}^{d} D_{t}, \\
\tilde{d}\left(k_{t} ; \xi_{t}\right)=\frac{A_{t}^{d}}{\tilde{\alpha}} k_{t}^{\tilde{\alpha}},
\end{gathered}
$$

where $\tilde{\sigma}, \nu>0, \tilde{\alpha} \in(0,1)$ and $\left\{\bar{C}_{t}, \bar{H}_{t}, \xi_{t}^{d}, A_{t}^{d}\right\}$ are bounded exogenous and positive disturbance processes which are among the exogenous disturbances included in the vector $\xi_{t}$. Our specification includes two housing related disturbances that will be of particular interest for our analysis, namely $\xi_{t}^{d}$ which captures shocks to housing preferences and $A_{t}^{d}$ shocks to the productivity in the construction of new houses. We impose linearity in the utility function (21) as this greatly facilitates the analytical characterization of optimal policy.

Each differentiated good is supplied by a single monopolistically competitive producer; there is a common technology for the production of all goods, in which (industry-specific) labor is the only variable input,

$$
y_{t}(i)=A_{t} f\left(h_{t}(i)\right)=A_{t} h_{t}(i)^{1 / \phi},
$$

where $A_{t}$ is an exogenously varying technology factor, and $\phi>1$. The Dixit-Stiglitz preferences (18) imply that the quantity demanded of each individual good $i$ will equal ${ }^{13}$

$$
y_{t}(i)=Y_{t}\left(\frac{p_{t}(i)}{P_{t}}\right)^{-\eta},
$$

where $Y_{t}$ is the total demand for the composite good defined in (18), $p_{t}(i)$ is the (money) price of the individual good, and $P_{t}$ is the price index,

$$
P_{t} \equiv\left[\int_{0}^{1} p_{t}(i)^{1-\eta} d i\right]^{\frac{1}{1-\eta}},
$$

corresponding to the minimum cost for which a unit of the composite good can be purchased in period $t$. Total demand is given by

$$
Y_{t}=C_{t}+k_{t}+g_{t} Y_{t}
$$

where $g_{t}$ is the share of the total amount of composite good purchased by the government, treated here as an exogenous disturbance process.

\footnotetext{
${ }^{13}$ In addition to assuming that household utility depends only on the quantity obtained of $C_{t}$, we assume that the government also cares only about the quantity obtained of the composite good defined by (18), and that it seeks to obtain this good through a minimum-cost combination of purchases of individual goods.
} 


\subsection{Household Optimality Conditions}

Each household maximizes utility by choosing state contingent sequences $\left\{C_{t}, H_{t}(j), D_{t}, k_{t}, B_{t}\right\}$ taking as given the process for $\left\{P_{t}, w_{t}(j), q_{t}, i_{t}, \Sigma_{t}, T_{t}\right\}$. The first order conditions give rise to an optimal labor supply relation

$$
w_{t}(j)=\frac{\tilde{v}_{H}\left(H_{t}(j) ; \xi_{t}\right)}{\tilde{u}_{C}\left(C_{t} ; \xi_{t}\right)}
$$

a consumption Euler equation

$$
\tilde{u}_{C}\left(C_{t} ; \xi_{t}\right)=\beta \widehat{E}_{t}\left[\tilde{u}_{C}\left(C_{t+1} ; \xi_{t+1}\right) \frac{1+i_{t}}{\Pi_{t+1}}\right],
$$

an equation characterizing optimal investment in new houses

$$
k_{t}=\left(\left(1+s^{d}\right) A_{t}^{d} q_{t}\right)^{\frac{1}{1-\tilde{\alpha}}}
$$

and an asset pricing equation

$$
q_{t}^{u}=\xi_{t}^{d}+\beta(1-\delta) \widehat{E}_{t} q_{t+1}^{u},
$$

where

$$
q_{t}^{u} \equiv q_{t} C_{t}^{-\tilde{\sigma}^{-1}} \bar{C}_{t}^{\tilde{\sigma}^{-1}}
$$

is the market valuation of housing in period $t$, expressed in marginal-utility units. The variable $q_{t}^{u}$ provides a measure of whether housing is currently expensive or inexpensive, in units that are particularly relevant for determining housing demand. More importantly, because of (30), it is expectations about the future value of $q_{T}^{u}$, rather than the future value of $q_{T}$ as such, that influence the current market value of housing, so that the degree of distortion that may be present in expectations regarding the former variable is of particular importance for equilibrium determination. The housing-price variable $q_{t}^{u}$ is accordingly of particular interest.

Equations (27)-(30) jointly characterize optimal household behavior under distorted beliefs. Using (26) and (29), one an express aggregate demand as

$$
Y_{t}=\frac{C_{t}+\Omega_{t} C_{t}^{\frac{\tilde{\sigma}^{-1}}{1-\tilde{\alpha}}}}{1-g_{t}}
$$

where

$$
\Omega_{t} \equiv\left(\left(1+s^{d}\right) A_{t}^{d} \bar{C}_{t}^{-\tilde{\sigma}^{-1}} q_{t}^{u}\right)^{\frac{1}{1-\tilde{\alpha}}}>0
$$

is a term that depends on exogenous shocks and belief distortions only.

\subsection{Optimal Price Setting by Firms}

The producers in each industry fix the prices of their goods in monetary units for a random interval of time, as in the model of staggered pricing introduced by Calvo (1983) and Yun (1996). Let $0 \leq \alpha<1$ be the fraction of prices that remain unchanged in any period. A supplier that changes its price in period $t$ chooses its new price $p_{t}(i)$ to maximize

$$
\widehat{E}_{t} \sum_{T=t}^{\infty} \alpha^{T-t} Q_{t, T} \Pi\left(p_{t}(i), p_{T}^{j}, P_{T} ; Y_{T}, q_{T}^{u}, \xi_{T}\right),
$$

where $\widehat{E}_{t}$ is the distorted expectations of price setters conditional on time $t$ information, which are assumed identical to the expectations held by consumers, $Q_{t, T}$ is the stochastic discount 
factor by which financial markets discount random nominal income in period $T$ to determine the nominal value of a claim to such income in period $t, \alpha^{T-t}$ is the probability that a price chosen in period $t$ will not have been revised by period $T$, and the function $\Pi\left(p_{t}(i), \ldots\right)$ indicates the nominal profits of the firm in period $t$ (discussed further below). In equilibrium, the discount factor is given by

$$
Q_{t, T}=\beta^{T-t} \frac{\tilde{u}_{C}\left(C_{T} ; \xi_{T}\right)}{\tilde{u}_{C}\left(C_{t} ; \xi_{t}\right)} \frac{P_{t}}{P_{T}} .
$$

Profits are equal to after-tax sales revenues net of the wage bill. Sales revenues are determined by the demand function (24), so that (nominal) after-tax revenue equals

$$
\left(1-\tau_{t}\right) p_{t}(i) Y_{t}\left(\frac{p_{t}(i)}{P_{t}}\right)^{-\eta}
$$

Here $\tau_{t}$ is a proportional tax on sales revenues in period $t ;\left\{\tau_{t}\right\}$ is treated as an exogenous disturbance process, taken as given by the monetary policymaker. We assume that $\tau_{t}$ fluctuates over a small interval around a non-zero steady-state level $\underline{\tau}$. We allow for exogenous variations in the tax rate in order to include the possibility of "pure cost-push shocks" that affect equilibrium pricing behavior while implying no change in the efficient allocation of resources.

The real wage demanded for labor of type $j$ is given by equation (27) and firms are assumed to be wage-takers. Because the right-hand side of (32) is a monotonically increasing function of $C_{t},(32)$ implies the existence of a differentiable function

$$
C_{t}=C\left(Y_{t}, q_{t}^{u}, \xi_{t}\right)
$$

solving (32) with the derivative $C_{Y}$ satisfying $0<C_{Y}\left(Y_{t}, q_{t}^{u}, \xi_{t}\right)<1-g$. Using this function and the assumed functional forms for preferences and technology, the nominal wage bill will equal

$$
\begin{aligned}
P_{t} w_{t}(j) h_{t}(i) & =P_{t} \frac{\lambda H_{t}(i)^{\nu} \bar{H}_{t}^{-\nu}}{C_{t}^{-\widetilde{\sigma}^{-1}} \bar{C}_{t}^{\widetilde{\sigma}^{-1}}} h_{t}(i) \\
& =\lambda P_{t}\left(\frac{p_{t}(i)}{P_{t}}\right)^{-\eta \phi}\left(\frac{p_{t}^{j}}{P_{t}}\right)^{-\eta \phi \nu} \bar{H}_{t}^{-\nu}\left(\frac{Y_{t}}{A_{t}}\right)^{1+\omega}\left(\frac{C\left(Y_{t}, q_{t}^{u}, \xi_{t}\right)}{\bar{C}_{t}}\right)^{\widetilde{\sigma}^{-1}}
\end{aligned}
$$

where

$$
\omega \equiv \phi(1+\nu)-1>0
$$

is the elasticity of real marginal cost in an industry with respect to industry output. Subtracting the nominal wage bill from the above expression for nominal after tax revenue, we obtain the function $\Pi\left(p_{t}(i), p_{T}^{j}, P_{T} ; Y_{T}, q_{T}^{u}, \xi_{T}\right)$ used in (34). The vector of exogenous disturbances $\xi_{t}$ now includes $A_{t}, g_{t}$ and $\tau_{t}$, in addition to the shocks $\left(\bar{C}_{t}, \bar{H}_{t}, \xi_{t}^{d}, A_{t}^{d}\right)$.

Each of the suppliers that revise their prices in period $t$ chooses the same new price $p_{t}^{*}$, that maximizes (34). Note that supplier $i$ 's profits in (34) are a concave function of the quantity sold $y_{t}(i)$, since revenues are proportional to $y_{t}(i)^{\frac{\eta-1}{\eta}}$ and hence concave in $y_{t}(i)$, while costs are convex in $y_{t}(i)$. Moreover, since $y_{t}(i)$ is proportional to $p_{t}(i)^{-\eta}$, the profit function is also concave in $p_{t}(i)^{-\eta}$. The first-order condition for the optimal choice of the price $p_{t}(i)$ is the same as the one with respect to $p_{t}(i)^{-\eta}$; hence the first-order condition with respect to $p_{t}(i)$,

$$
\widehat{E}_{t} \sum_{T=t}^{\infty} \alpha^{T-t} Q_{t, T} \Pi_{1}\left(p_{t}(i), p_{T}^{j}, P_{T} ; Y_{T}, q_{T}^{u}, \xi_{T}\right)=0
$$


is both necessary and sufficient for an optimum. The equilibrium choice $p_{t}^{*}$ (which is the same for each firm in industry $j$ ) is the solution to the equation obtained by substituting $p_{t}(i)=p_{t}^{j}=p_{t}^{*}$ into the above first-order condition.

Under the assumed isoelastic functional forms, the optimal choice has a closed-form solution

$$
\frac{p_{t}^{*}}{P_{t}}=\left(\frac{K_{t}}{F_{t}}\right)^{\frac{1}{1+\omega \eta}}
$$

where $F_{t}$ and $K_{t}$ capture the effects of discounted marginal costs and revenues, respectively, and are defined by

$$
\begin{aligned}
& F_{t} \equiv \widehat{E}_{t} \sum_{T=t}^{\infty}(\alpha \beta)^{T-t} f\left(Y_{T}, q_{T}^{u}, \xi_{T}\right)\left(\frac{P_{T}}{P_{t}}\right)^{\eta-1}, \\
& K_{t} \equiv \widehat{E}_{t} \sum_{T=t}^{\infty}(\alpha \beta)^{T-t} k\left(Y_{T}, \xi_{T}\right)\left(\frac{P_{T}}{P_{t}}\right)^{\eta(1+\omega)},
\end{aligned}
$$

where

$$
\begin{aligned}
f\left(Y, q^{u} ; \xi\right) & \equiv(1-\tau) \bar{C}^{\tilde{\sigma}^{-1} Y} C\left(Y, q^{u}, \xi\right)^{-\widetilde{\sigma}^{-1}}, \\
k(Y ; \xi) & \equiv \frac{\eta}{\eta-1} \lambda \phi \frac{\bar{H}^{-\nu}}{A^{1+\omega}} Y^{1+\omega}
\end{aligned}
$$

Relations (38)-(39) can also be written in the recursive form

$$
\begin{aligned}
F_{t} & =f\left(Y_{t}, q_{t}^{u} ; \xi_{t}\right)+\alpha \beta \widehat{E}_{t}\left[\Pi_{t+1}^{\eta-1} F_{t+1}\right] \\
K_{t} & =k\left(Y_{t} ; \xi_{t}\right)+\alpha \beta \widehat{E}_{t}\left[\Pi_{t+1}^{\eta(1+\omega)} K_{t+1}\right]
\end{aligned}
$$

where $\Pi_{t} \equiv P_{t} / P_{t-1} \cdot{ }^{14}$ The price index then evolves according to a law of motion

$$
P_{t}=\left[(1-\alpha) p_{t}^{* 1-\eta}+\alpha P_{t-1}^{1-\eta}\right]^{\frac{1}{1-\eta}},
$$

as a consequence of (25). Substitution of (37) into (44) implies that equilibrium inflation in any period is given by

$$
\frac{1-\alpha \Pi_{t}^{\eta-1}}{1-\alpha}=\left(\frac{F_{t}}{K_{t}}\right)^{\frac{\eta-1}{1+\omega \eta}} .
$$

Equations (42), (43) and (45) jointly define a short-run aggregate supply relation between inflation, output and house prices, given the current disturbances $\xi_{t}$, and (potentially distorted) expectations regarding future inflation, output, house prices and disturbances.

\subsection{Summary and Equilibrium Definition}

For the subsequent analysis it will be helpful to express the model in terms of the endogenous variables $\left(Y_{t}, K_{t}, F_{t}, \Delta_{t}, q_{t}^{u}, m_{t}, i_{t}\right)$ only, where $m_{t}$ is the belief distortions of the private sector and

$$
\Delta_{t} \equiv \int_{0}^{1}\left(\frac{p_{t}(i)}{P_{t}}\right)^{-\eta(1+\omega)} d i \geq 1
$$

\footnotetext{
${ }^{14}$ It is evident that (38) implies (42); but one can also show that processes that satisfy (42) each period, together with certain bounds, must satisfy (38). Since we are interested below only in the characterization of bounded equilibria, we can omit the statement of the bounds that are implied by the existence of well-behaved expressions on the right-hand sides of (38) and (39), and treat (42)-(43) as necessary and sufficient for processes $\left\{F_{t}, K_{t}\right\}$ to measure the relevant marginal conditions for optimal price-setting.
} 
a measure of price dispersion at time $t$. The vector of exogenous disturbances is given by $\xi_{t}=\left(A_{t}, g_{t}, \tau_{t}, \bar{C}_{t}, \bar{H}_{t}, \xi_{t}^{d}, A_{t}^{d}\right)^{\prime}$.

We begin by expressing expected household utility (evaluated under the objective measure $\mathcal{P}$ ) in terms of these variables. Inverting the production function (23) to write the demand for each type of labor as a function of the quantities produced of the various differentiated goods, it is possible to write the utility of the representative household as a function of the expected production plan $\left\{y_{t}(i)\right\}$. One thereby obtains

$$
U \equiv E_{0} \sum_{t=0}^{\infty} \beta^{t}\left[u\left(Y_{t}, q_{t}^{u} ; \xi_{t}\right)-\int_{0}^{1} v\left(y_{t}^{j} ; \xi_{t}\right) d j+\tilde{\omega}\left(D_{t} ; \xi_{t}\right)\right],
$$

with

$$
\begin{aligned}
u\left(Y_{t}, q_{t}^{u} ; \xi_{t}\right) & \equiv \tilde{u}\left(C\left(Y_{t}, q_{t}^{u}, \xi_{t}\right) ; \xi_{t}\right) \\
v\left(y_{t}^{j} ; \xi_{t}\right) & \equiv \tilde{v}\left(f^{-1}\left(y_{t}^{j} / A_{t}\right) ; \xi_{t}\right)
\end{aligned}
$$

where in this last expression we make use of the fact that the quantity produced of each good in industry $j$ will be the same, and hence can be denoted $y_{t}^{j}$; and that the quantity of labor hired by each of these firms will also be the same, so that the total demand for labor of type $j$ is proportional to the demand of any one of these firms.

One can furthermore express the relative quantities demanded of the differentiated goods each period as a function of their relative prices, using (24). This and the linear dependence of utility on the stock of assets allows us to write the utility flow to the representative household in the form

$$
u\left(Y_{t}, q_{t}^{u} ; \xi_{t}\right)-v\left(Y_{t} ; \xi_{t}\right) \Delta_{t}+\bar{\xi}_{t}^{d} \frac{A_{t}^{d}}{\tilde{\alpha}} k_{t}^{\tilde{\alpha}},
$$

where

$$
\bar{\xi}_{t}^{d} \equiv \sum_{T=t}^{\infty} E_{t}\left[(1-\delta)^{T-t} \beta^{T-t} \xi_{T}^{d}\right]
$$

We can use (29), (31) and (36) to express $k_{t}$ in terms of $Y_{t}, q_{t}^{u}$ and exogenous shocks. Hence we can express the household objective (46) as

$$
U=E_{0} \sum_{t=0}^{\infty} \beta^{t} U\left(Y_{t}, \Delta_{t}, q_{t}^{u} ; \xi_{t}\right)
$$

where the explicit expression for the flow utility is given by

$$
\begin{aligned}
U\left(Y_{t}, \Delta_{t}, q_{t}^{u} ; \xi_{t}\right) & =\frac{\bar{C}_{t}^{\tilde{\sigma}^{-1}} C\left(Y_{t}, q_{t}^{u}, \xi_{t}\right)^{1-\tilde{\sigma}^{-1}}}{1-\tilde{\sigma}^{-1}} \\
& -\frac{\lambda}{1+\nu} \bar{H}_{t}^{-\nu}\left(\frac{Y_{t}}{A_{t}}\right)^{1+\omega} \Delta_{t} \\
& +\frac{A_{t}^{d} \bar{\xi}_{t}^{d}}{\tilde{\alpha}} \Omega\left(q_{t}^{u}, \xi_{t}\right)^{\tilde{\alpha}} C\left(Y_{t}, q_{t}^{u}, \xi_{t}\right)^{\frac{\tilde{\alpha}}{1-\tilde{\alpha}} \tilde{\sigma}^{-1}}
\end{aligned}
$$

which is a monotonically decreasing function of $\Delta$ given $Y, q^{u}$ and $\xi$ and where $\Omega\left(q_{t}^{u}, \xi_{t}\right)$ is the function defined in (33).

The consumption Euler equation (28) can be expressed as

$$
\tilde{u}_{C}\left(C\left(Y_{t}, q_{t}^{u}, \xi_{t}\right) ; \xi_{t}\right)=\beta \widehat{E}_{t}\left[\tilde{u}_{C}\left(C\left(Y_{t}, q_{t}^{u}, \xi_{t}\right) ; \xi_{t+1}\right) \frac{1+i_{t}}{\Pi_{t+1}}\right]
$$


Using (45) to substitute for the variable $\Pi_{t}$ equations (42) and (43) can be expressed as

$$
\begin{aligned}
F_{t} & =f\left(Y_{t}, q_{t}^{u} ; \xi_{t}\right)+\alpha \beta \widehat{E}_{t}\left[\phi_{F}\left(K_{t+1}, F_{t+1}\right)\right] \\
K_{t} & =k\left(Y_{t} ; \xi_{t}\right)+\alpha \beta \widehat{E}_{t}\left[\phi_{K}\left(K_{t+1}, F_{t+1}\right)\right],
\end{aligned}
$$

where the functions $\phi_{F}, \phi_{K}$ are both homogeneous degree 1 functions of $K$ and $F$. The system consisting of (51)-(52) can be written more compactly as

$$
Z_{t}=z\left(Y_{t}, q_{t}^{u} ; \xi_{t}\right)+\alpha \beta \widehat{E}_{t}\left[\Phi\left(Z_{t+1}\right)\right]
$$

where

$$
Z_{t} \equiv\left[\begin{array}{c}
F_{t} \\
K_{t}
\end{array}\right], \quad z\left(Y, q^{u} ; \xi\right) \equiv\left[\begin{array}{c}
f\left(Y, q^{u} ; \xi\right) \\
k(Y ; \xi)
\end{array}\right], \quad \Phi(Z) \equiv\left[\begin{array}{c}
\phi_{F}(K, F) \\
\phi_{K}(K, F)
\end{array}\right] .
$$

Because the relative prices of the industries that do not change their prices in period $t$ remain the same, one can use (44) to derive a law of motion for the price dispersion term $\Delta_{t}$ of the form

$$
\Delta_{t}=h\left(\Delta_{t-1}, \Pi_{t}\right)
$$

where

$$
h(\Delta, \Pi) \equiv \alpha \Delta \Pi^{\eta(1+\omega)}+(1-\alpha)\left(\frac{1-\alpha \Pi^{\eta-1}}{1-\alpha}\right)^{\frac{\eta(1+\omega)}{\eta-1}} .
$$

This is the source of welfare losses from inflation or deflation. Using once more (45) to substitute for the variable $\Pi_{t}$ one obtains

$$
\Delta_{t}=\tilde{h}\left(\Delta_{t-1}, K_{t} / F_{t}\right) .
$$

The asset pricing equation (30) and equations (50)-(56) represent five constraints on the equilibrium paths of the seven endogenous variables $\left(Y_{t}, F_{t}, K_{t}, \Delta_{t}, q_{t}^{u}, m_{t+1}, i_{t}\right)$. For a given sequence of belief distortions $m_{t}$ satisfying restriction (10) there is thus one degree of freedom left, which can be determined by monetary policy.

We are now in a position to define the equilibrium with distorted private sector expectations:

Definition 1 (DEE) A distorted expectations equilibrium (DEE) is a bounded stochastic process for $\left\{Y_{t}, F_{t}, K_{t}, \Delta_{t}, q_{t}^{u}, m_{t+1}, i_{t}\right\}_{t=0}^{\infty}$ satisfying equations (10), (30) and (50)-(56).

We can also define the following special case considered in the standard literature on optimal monetary policy:

Definition 2 (REE) A rational expectations equilibrium (REE) is a bounded stochastic process for $\left\{Y_{t}, F_{t}, K_{t}, \Delta_{t}, q_{t}^{u}, i_{t}\right\}_{t=0}^{\infty}$ satisfying equations (30) and (50)-(56) with $m_{t+1} \equiv 1$ in all states.

\section{Optimal Policy under Rational Expectations}

We first consider the nature of an optimal policy commitment under the assumption of rational expectations. We will then consider the more general case allowing for belief distortions as a perturbation of our solution for this case.

Under the assumption of rational expectations, equation (30) has a unique bounded solution given by

$$
q_{t}^{u}=\bar{\xi}_{t}^{d}
$$

for all $t$. Thus the housing price is necessarily equal to its fundamental value $\bar{\xi}_{t}^{d}$ regardless of policy. Note that $\bar{\xi}_{t}^{d}$, defined in equation (47), is purely a function of exogenous disturbances. Thus under the assumption of rational expectations, we can treat $q_{t}^{u}$ as an exogenous disturbance, rather than an additional endogenous variable. Our model with a housing sector then becomes equivalent to a standard New Keynesian model, except with additional sources of exogenous disturbances. 


\subsection{Optimal Dynamics under Commitment}

An optimal policy commitment will be a rule that results in an evolution of the endogenous variables that maximizes (48) subject to the constraints that (53) and (56) hold for each $t \geq 0$, and an initial precommitment specifying a value for a linear combination of $\Phi\left(Z_{0}\right)$ and $q_{0}^{u}$, chosen so as to allow us to obtain a time-invariant system of first-order conditions. Note that we need not list equation (50) as a constraint on the problem. Under the assumption that the zero lower bound on nominal interest rates is not binding, constraint (50) imposes no restrictions on the path of the other variables. ${ }^{15}$ The required path for nominal interest rates can then be computed ex-post using the solution for the other variables and equation (50).

The first-order conditions of this planning problem are given by

$$
\begin{aligned}
U_{Y}\left(Y_{t}, \Delta_{t}, q_{t}^{u} ; \xi_{t}\right)+\Gamma_{t}^{\prime} z_{Y}\left(Y_{t}, q_{t}^{u}, \xi_{t}\right) & =0 \\
-\gamma_{t} \tilde{h}_{2}\left(\Delta_{t-1}, K_{t} / F_{t}\right) \frac{K_{t}}{F_{t}^{2}}-\Gamma_{1 t}+\alpha \Gamma_{t-1}^{\prime} D_{1}\left(K_{t} / F_{t}\right) & =0 \\
\gamma_{t} \tilde{h}_{2}\left(\Delta_{t-1}, K_{t} / F_{t}\right) \frac{1}{F_{t}}-\Gamma_{2 t}+\alpha \Gamma_{t-1}^{\prime} D_{2}\left(K_{t} / F_{t}\right) & =0 \\
U_{\Delta}\left(Y_{t}, \Delta_{t}, q_{t}^{u} ; \xi_{t}\right)-\gamma_{t}+\beta E_{t}\left[\gamma_{t+1} \tilde{h}_{1}\left(\Delta_{t}, K_{t+1} / F_{t+1}\right)\right] & =0
\end{aligned}
$$

for all $t \geq 0$. The Lagrange multiplier vector $\Gamma_{t}$ is associated with constraints (51) and (52) and has elements $\Gamma_{t}^{\prime}=\left(\Gamma_{1 t}, \Gamma_{2, t}\right)$. The multiplier $\gamma_{t}$ is associated with constraint (56). The firstorder conditions take this form also for $t=0$ on the assumption that the initial precommitment specifies a value for

$$
\alpha \Gamma_{-1}^{\prime} \Phi\left(Z_{0}\right)+(1-\delta) \Psi_{-1} q_{0}^{u}
$$

for some multipliers $\Gamma_{-1}$ and $\Psi_{-1}$.

Above, $\tilde{h}_{i}(\Delta, K / F)$ denotes the partial derivative of $\tilde{h}(\Delta, K / F)$ with respect to its $i$-th argument, and $D_{i}(K / F)$ is the $i$-th column of the matrix

$$
D(Z) \equiv\left[\begin{array}{cc}
\partial_{F} \phi_{F}(Z) & \partial_{K} \phi_{F}(Z) \\
\partial_{F} \phi_{K}(Z) & \partial_{K} \phi_{K}(Z)
\end{array}\right]
$$

Since the elements of $\Phi(Z)$ are homogeneous degree 1 functions of $Z$, the elements of $D(Z)$ are all homogenous degree 0 functions of $Z$, and hence functions of $K / F$ only. Thus we can alternatively write $D(K / F)$.

The optimal dynamics are then bounded stochastic processes $\left\{Y_{t}, F_{t}, K_{t}, \Delta_{t}\right\}$ that satisfy the structural equations (51)-(56) and the first order conditions (58)-(61), together with the initial pre-commitments.

\subsection{The Second-Best Steady State}

We shall be concerned solely with optimal outcomes that involve small fluctuations around a deterministic state. The considered deterministic steady state is the one associated with an optimal monetary policy, taking as given the real distortions (such as the housing subsidy and monopolistic competition) that monetary policy by itself cannot eliminate. We call this steady state the second-best steady state and it is defined as follows:

Definition 3 The second-best steady state is a set of constant values $\left(\underline{Y}, \underline{Z}, \underline{\Delta}, \underline{q^{u}}, \underline{\gamma}, \underline{\Gamma}\right)$ that solve the structural equations (30),(51)-(56) and the FOCs (58)-(61) in the case that $\bar{\xi}_{t}=\underline{\xi}$ at all times and initial conditions consistent with the steady state are assumed.

\footnotetext{
${ }^{15}$ This assertion also depends on our assumption here that the central bank chooses its interest-rate operating target $i_{t}$ with full information about the state of the economy at date $t$.
} 
We now compute this steady state. Equation (30) implies $\underline{q}^{u}=\underline{\bar{\xi}}^{d}$. Moreover, as in the model without housing, considered in Adam and Woodford (2012), the second-best steady state satisfies $\underline{F}=\underline{K}=(1-\alpha \beta)^{-1} k(\underline{Y}, \underline{\xi})$, which implies $\underline{\Pi}=1$ (no inflation) and $\underline{\Delta}=1$ (zero price dispersion), where the value of $\underline{Y}$ is implicitly defined by

$$
f\left(\underline{Y}, \underline{q^{u}}, \underline{\xi}\right)=k(\underline{Y}, \underline{\xi}) .
$$

As shown in appendix A.1, there exists a unique steady state consumption level $\underline{Y}$ solving (64).

Furthermore, with $\tilde{h}_{2}(1,1)=0$ (the effects of a small non-zero inflation rate on the measure of price dispersion are of second order), conditions (59)-(60) reduce in the steady state to the eigenvector condition

$$
\underline{\Gamma}^{\prime}=\alpha \underline{\Gamma^{\prime}} D(1) .
$$

Moreover, since when evaluated at a point where $F=K$,

$$
\frac{\partial \log \left(\phi_{K} / \phi_{F}\right)}{\partial \log K}=-\frac{\partial \log \left(\phi_{K} / \phi_{F}\right)}{\partial \log F}=\frac{1}{\alpha},
$$

we observe that $D(1)$ has a left eigenvector $[1-1]$, with eigenvalue $1 / \alpha$; hence $(65)$ is satisfied if and only if $\underline{\Gamma}_{2}=-\underline{\Gamma}_{1}$.

Condition (58) provides one additional condition

$$
U_{Y}\left(\underline{Y}, 1, \underline{q}^{u} ; \underline{\xi}\right)+\underline{\Gamma}_{1}\left(f_{Y}\left(\underline{Y}, \underline{q}^{u}, \underline{\xi}\right)-k_{Y}\left(\underline{Y}, \underline{q}^{u}, \underline{\xi}\right)\right)=0,
$$

which uniquely determines $\underline{\Gamma}_{1}$, given the solutions for $\underline{Y}$ and $\underline{q}^{u}$. Appendix A.1 shows that

$$
k_{Y}-f_{Y}>0
$$

so that $\underline{\Gamma}_{1}$ has the same sign as $U_{Y}$, the sign of which is discussed below.

Condition (61) provides a restriction that determines the steady state value of $\underline{\gamma}$ :

$$
U_{\Delta}\left(\underline{Y}, 1, \underline{q}^{u} ; \underline{\xi}\right)-\underline{\gamma}+\beta \underline{\gamma} \tilde{h}_{1}(1,1)=0 .
$$

Since $U_{\Delta}<0$ and $\tilde{h}_{1}(1,1)=\alpha$, we have

$$
\underline{\gamma}=\frac{U_{\Delta}\left(\underline{Y}, 1, \underline{q}^{u} ; \underline{\xi}\right)}{(1-\beta \alpha)}<0 .
$$

\subsection{Comparison with the Optimal Steady State}

The optimality of the equilibrium level of output can be assessed by evaluating $U_{Y}$. In the second-best steady state, appendix A.1 proves that

$$
\begin{aligned}
& U_{Y}\left(\underline{Y}, 1, \underline{q}^{u} ; \underline{\xi}\right)= \\
& \quad \bar{C}^{\tilde{\sigma}^{-1}} C\left(\underline{Y}, \underline{q}^{u}, \underline{\xi}\right)^{-\tilde{\sigma}^{-1}}\left(\frac{1-g+s^{d} C_{Y}\left(\underline{Y}, \underline{q}^{u}, \underline{\xi}\right)}{1+s^{d}}-\frac{\eta-1}{\eta}(1-\underline{\tau})\right) .
\end{aligned}
$$

This shows that in the absence of a housing subsidy $\left(s^{d}=0\right)$, we have $U_{Y}=0$ and thus $\underline{\Gamma}_{1}=0$, if and only if the output subsidy eliminates the steady state monopoly distortion, i.e., when the wedge $\Theta=0$, where

$$
\Theta \equiv \log \frac{\eta}{\eta-1} \frac{1-\underline{g}}{1-\underline{\tau}}
$$

We refer to the steady state in which steady-state distortions are absent as the optimal steady state: 
Definition 4 The optimal steady state $\left(\underline{Y}^{*}, \underline{Z}^{*}, \underline{\Delta}^{*}, \underline{q}^{u *}, \underline{\gamma}^{*}, \underline{\Gamma}^{*}\right)$ is a set constant values of our endogenous variables associated with a second-best steady state in which $s^{d}=0$ and $\Theta=0$.

We will locally approximate our structural equations and household welfare around this optimal steady state. More generally, we shall consider the case with a non-zero housing subsidy/tax. Conditional on the value of housing subsidy $s^{d} \lessgtr 0$ one can then define an efficient steady state output subsidy $\underline{\tau}^{\text {eff }}\left(s^{d}\right)$, which is the value of $\underline{\tau}$ such that $U_{Y}=0$ in (67). ${ }^{16}$ Appendix A.2 then establishes the following result:

Lemma 1 Given a housing subsidy $s^{d} \lessgtr 0$ and the efficient output subsidy $1-\underline{\tau}=1-\underline{\tau}^{\text {eff }}\left(s^{d}\right)$, we have

$$
U_{Y}\left(\underline{Y}, 1, \underline{q}^{u} ; \underline{\xi}\right)=\underline{\Gamma}_{1}=0 .
$$

If the output subsidy falls short of its efficient value, $1-\underline{\tau}<1-\underline{\tau}^{\text {eff }}\left(s^{d}\right)$, then

$$
U_{Y}\left(\underline{Y}, 1, \underline{q}^{u} ; \underline{\xi}\right)>0, \underline{\Gamma}_{1}>0 .
$$

If instead $1-\underline{\tau}>1-\underline{\tau}^{\text {eff }}\left(s^{d}\right)$, one obtains

$$
U_{Y}\left(\underline{Y}, 1, \underline{q}^{u} ; \underline{\xi}\right)<0, \underline{\Gamma}_{1}<0 .
$$

The previous lemma shows that the marginal utility of output is positive (negative) in the second-best steady state, whenever the output subsidy falls short of (exceeds) the output subsidy that would be efficient given the actual level of housing subsidies.

We can also consider the effect of perturbations to the asset pricing equation (30). If we treat $q_{t}^{u}$ as an endogenous variable in the planning problem considered in section 4.1, and equation (30) as an additional constraint, we obtain the additional first order condition

$$
U_{q^{u}}\left(Y_{t}, \Delta_{t}, q_{t}^{u} ; \xi_{t}\right)+\Gamma_{t}^{\prime} z_{q^{u}}\left(Y_{t}, q_{t}^{u} ; \xi_{t}\right)+\Psi_{t-1}(1-\delta)-\Psi_{t}=0
$$

for all $t \geq 0$, where $\Psi_{t}$ is the Lagrange multiplier associated with constraint (30). In the steady state, equation (69) implies

$$
\underline{\Psi}=\frac{1}{\delta}\left(U_{q^{u}}\left(\underline{Y}, 1, \underline{q}^{u} ; \underline{\xi}\right)+\underline{\Gamma}_{1} f_{q^{u}}\left(\underline{Y}, \underline{q}^{u} ; \underline{\xi}\right)\right) .
$$

Appendix A.3 then proves the following result:

Lemma 2 If $s^{d}=0, U_{q^{u}}\left(\underline{Y}, 1, \underline{q}^{u} ; \underline{\xi}\right)=0$. If $s^{d}>0\left(s^{d}<0\right)$, then $U_{q^{u}}<0\left(U_{q^{u}}>0\right)$. Since $\underline{\Gamma}_{1}=0$ for $\underline{\tau}=\underline{\tau}^{\text {eff }}\left(s^{d}\right)$, it follows that

$$
\begin{aligned}
& \underline{\Psi}=0 \quad \text { if } s^{d}=0 \text { and } \underline{\tau}=\tau^{\text {eff }}(0) \\
& \underline{\Psi}<0 \quad \text { if } s^{d}>0 \text { and } \underline{\tau} \text { is sufficiently close to } \underline{\tau}^{\text {eff }}\left(s^{d}\right) \\
& \underline{\Psi}>0 \quad \text { if } s^{d}<0 \text { and } \underline{\tau} \text { is sufficiently close to } \underline{\tau}^{\text {eff }}\left(s^{d}\right)
\end{aligned}
$$

This shows that for a positive housing subsidy, the representative household's utility is decreasing with further house price increases, whenever the output subsidy is sufficiently close to its efficient level. Correspondingly, in the presence of a housing tax, household utility decreases with a fall in housing prices. Intuitively, holding the level of total output $Y$ fixed, an increase in the housing price leads to a further increase in housing investment, which is already inefficiently high (low) when there is a housing subsidy (tax). House price changes due to distorted expectations will have welfare effects of this kind as in the case of any other perturbation of equation (30).

\footnotetext{
${ }^{16}$ Note that implementing the efficient steady state substidiy $\underline{\tau}^{\text {eff }}$ does result in an efficient steady state outcome when $s^{d} \neq 0$.
} 


\subsection{Local Approximation of Optimal Dynamics}

We can now characterize equilibrium dynamics under an optimal policy commitment by linearizing equations (58)-(61) around the optimal steady state.

It is useful to write these linearized equations in terms of an output gap that measures the deviation of equilibrium output from its welfare maximizing level. Let us consider the allocation defined by $\left\{Y_{t}, q_{t}^{u}\right\}_{t=0}^{\infty}$ that maximizes the utility of the representative household, taking as given the equilibrium response of housing supply to any housing price sequence. In any period $t$, we define $\left(Y_{t}^{*}, q_{t}^{u *}\right)$ as the values $\left(Y_{t}, q_{t}^{u}\right)$ that maximize $U\left(Y_{t}, 1, q_{t}^{u} ; \xi_{t}\right)$. These are implicitly defined by

$$
U_{Y}\left(Y_{t}^{*}, 1, q_{t}^{u *} ; \xi_{t}\right)=U_{q^{u}}\left(Y_{t}^{*}, 1, q_{t}^{u *} ; \xi_{t}\right)=0 .
$$

Appendix A.4 shows that $U_{Y Y}<0, U_{Y q^{u}}=0$ and $U_{q^{u} q^{u}}<0$ at the optimal steady state, so that the second-order conditions for optimality are also satisfied for allocations close enough to the optimal steady state. We also show in appendix A.4 that

$$
q_{t}^{u *}=\frac{\bar{\xi}_{t}^{d}}{1+s^{d}},
$$

which implies that if $s^{d}>0$, the equilibrium housing price under rational expectations, given in equation (57), is higher than the optimal level at all times.

One can then derive a first-order approximation of the optimal dynamics for the variables

$$
\begin{aligned}
\pi_{t} & \equiv \log \Pi_{t} \\
y_{t}^{g a p} & =\log Y_{t}-\log Y_{t}^{*}
\end{aligned}
$$

where $y_{t}^{g a p}$ denotes the output gap.

Our local approximation applies to the case of exogenous disturbances that are small in the following sense. We assume linear dynamics for the vector of exogenous disturbances $\xi_{t}$ of the form

$$
\xi_{t}=E_{0} \xi_{t}+\sigma \sum_{k} \sum_{j=0}^{t-1} \xi_{j, k} e_{k, t-j},
$$

where $\left\{E_{0} \xi_{t}\right\}_{t=0}^{\infty}$ is a deterministic sequence satisfying $E_{0} \xi_{t}-\underline{\xi} \sim O(\sigma)$ for all $t \geq 0$; the $e_{k t}$ are a set of independent underlying disturbance processes, each assumed to be an i.i.d. random variable with distribution $N(0,1)$; the $\xi_{j, k}$ are vectors of coefficients; and $\sigma \geq 0$ is an expansion parameter. We fix the coefficients $\xi_{j, k}$ but consider the limiting case in which the parameter $\sigma$ is small. We also assume that steady state distortions are small in the sense that

$$
s^{d} \sim O(\sigma) \text { and } \Theta \sim O(\sigma),
$$

the initial price dispersion $\Delta_{-1}$ is small $\left(\Delta_{-1} \sim O\left(\sigma^{2}\right)\right)$, and the initial precommitment $(62)$ involves multipliers $\Gamma_{-1}, \Psi_{-1}$ of order $O(\sigma)$.

Under these assumptions, the second-best steady state corresponding to the non-zero values for $s^{d}$ and $\Theta$ involves $\underline{\Gamma}$ and $\underline{\Psi}$ of order $O(\sigma)$, as well as a steady state value for the output gap $x_{t}$ of order $O(\sigma)$. The second-best optimal dynamics will involve deviations of the variables from their steady state values that will also be of order $O(\sigma)$. To this first order of approximation, we can characterize the second-best optimal dynamics by log-linearizing equilibrium conditions (53), (56), and (58)-(61) around the constant values associated with the optimal steady state. 


\subsection{The Irrelevance of Housing Prices under Rational Expectations}

Under the assumption of an initial precommitment satisfying $\Gamma_{1,-1}=-\Gamma_{2,-1}$, the second-best optimal dynamics involve $\Gamma_{1, t}=-\Gamma_{2, t}$ for all $t \geq 0$ as well, allowing us to characterize the second-best optimal dynamics of inflation and the output gap $\left(\pi_{t}, y_{t}^{g a p}\right)$ without reference to any other endogenous variables. The evolution of these two variables is given by the unique bounded solution to the pair of linearized equations ${ }^{17}$

$$
\begin{aligned}
& \pi_{t}=\beta E_{t} \pi_{t+1}+\kappa_{y} y_{t}^{g a p}+u_{t} \\
& \Lambda_{\pi} \pi_{t}+\frac{\Lambda_{y}}{\kappa_{y}}\left(y_{t}^{g a p}-y_{t-1}^{g a p}\right)=0,
\end{aligned}
$$

where the constants $\kappa_{y}>0, \Lambda_{\pi}>0$ and $\Lambda_{y}>0$ are functions of the deep model parameters. (An explicit expression for $\kappa_{y}$ is provided in Appendix A.5, while Appendix A.6 derives $\Lambda_{\pi}$ and $\Lambda_{y}$.) The cost-push disturbance in equation (75) is given by

$$
u_{t}=\frac{(1-\alpha)(1-\alpha \beta)}{\alpha(1+\omega \eta)}\left(\Theta+\widehat{\tau}_{t}-\widehat{g}_{t}\right)
$$

where

$$
\begin{aligned}
& \widehat{\tau} \equiv-\log \left(\frac{1-\tau_{t}}{1-\underline{\tau}}\right) \\
& \widehat{g} \equiv-\log \left(\frac{1-g_{t}}{1-\underline{g}}\right)
\end{aligned}
$$

define deviations of $\tau_{t}$ and $g_{t}$ from their second-best steady state values.

Equation (75) indicates the form taken by the New Keynesian Phillips curve in our model. The presence of the housing sector makes no difference to this equation, when written in terms of inflation and the output gap, though housing variables, i.e., housing supply shocks $A_{t}^{d}$ and housing demand shocks $\xi_{t}^{d}$, do affect the definition of the target output level $Y_{t}^{*}$ and hence the definition of the output gap.

Equation (76) represents a target criterion for optimal monetary policy. This equation also involves only inflation and the output gap and is unaffected by any of the housing variables (except again insofar as they are involved in the definition of the output gap); in fact the criterion is identical to the optimal target criterion for a model without a housing sector. It immediately follows that the second-best optimal dynamics of inflation do not respond to shocks to housing supply or demand, nor do the second-best optimal dynamics of the output gap. Not only is it possible to formulate a criterion for optimal policy that makes no reference to housing prices, but none of the equilibrium determinants of housing prices affect this criterion, either. Thus under the assumption of rational expectations it would be appropriate for the monetary authority to determine a path for inflation that would not respond in any way to the occurrence of a housing boom.

\section{$5 \quad$ Linear Dynamics in the Presence of Belief Distortions}

We next consider how the equilibrium dynamics of endogenous variables are affected by belief distortions. We begin by observing that the only private sector expectations that matter for

\footnotetext{
${ }^{17}$ Equations (75) and (76) are a special case of the ones derived below for the more general case with belief distortions. Given the signs established for the coefficients $\kappa_{y}, \Lambda_{\pi}$ and $\Lambda_{y}$ and the fact that $\beta \in(0,1)$, the existence of a unique bounded solution follows from the same argument as in the model without a housing sector, see section 5.3 in Adam and Woodford (2012).
} 
the determination of endogenous variables under any policy are the subjective expectations $\widehat{E}_{t}\left[\Phi\left(Z_{t+1}\right)\right]$ that enter equation (53) and $\widehat{E}_{t}\left[q_{t+1}^{u}\right]$ that enter equation (30). It follows that under any policy, the worst-case beliefs will be of the form (15), where

$$
x_{t+1} \equiv\left(\begin{array}{c}
\Phi\left(Z_{t+1}\right) \\
q_{t+1}^{u}
\end{array}\right) .
$$

The multipliers $\left\{\zeta_{t}\right\}_{t=0}^{\infty}$ appearing in (15) can then be used to parameterize possible belief distortions.

We are again interested in a local approximation of the dynamics of first order in the expansion parameter $\sigma$, where the vector of exogenous disturbances $\xi_{t}$ is assumed to evolve according to (73). In addition to assuming only small fluctuations in the exogenous disturbances, we assume that the belief distortion process $\left\{\zeta_{t}\right\}_{t=0}^{\infty}$ involves fluctuations only of order $O(\sigma)$ around some constant vector $\underline{\zeta}$; we later verify that the worst case beliefs are indeed of this form, for a vector $\zeta$ that remains to be determined. We similarly assume that the Lagrange multipliers $\left\{\Gamma_{t}, \Psi_{t}\right\}_{t=0}^{\infty}$ are of order $O(\sigma)$, as in the rational expectations case, and that $\left\{\gamma_{t}\right\}_{t=0}^{\infty}$ differs from its optimal steady state value $\gamma^{*}$ only by a term of order $O(\sigma)$; we later verify that this is indeed the case. As in section 4.4, we also assume that steady state distortions are small, in the sense that $s^{d} \sim O(\sigma)$ and $\Theta \sim O(\sigma)$, that the initial price dispersion $\Delta_{-1}$ is small $\left(\Delta_{-1} \sim O\left(\sigma^{2}\right)\right)$, and that the initial precommitment (62) involves multipliers $\Gamma_{-1}, \Psi_{-1}$ of order $O(\sigma)$.

We also assume that the parameter $\theta$, measuring the degree of concern for robustness to belief distortions, is small in the sense that

$$
\theta \sim O\left(\sigma^{2}\right)
$$

Under this assumption, the belief distortion factor $m_{t+1}$ implied by (15) will continue to vary across states to a non-trivial extent, even in the limit as $\sigma$ is made arbitrarily small. This assumption allows for more significant belief distortions in the case of small exogenous disturbances than in the analysis in Adam and Woodford (2012), where $\theta$ was assumed to have a fixed positive value regardless of the magnitude of $\sigma$, so that the worst-case fluctuations in $m_{t+1}$ were only of order $O(\sigma)$. In our earlier paper, this implied that the effects of belief distortions on the model's structural equations were at most of order $O\left(\sigma^{2}\right)$. Here, instead, we obtain linear approximations to the model structural equations that include belief distortion terms of order $O(\sigma)$. In particular, we find that the worst-case belief distortions will generally imply mis-pricing of housing of order $O(\sigma)$.

We look for solutions to the structural equations in which the log deviations

$$
w_{t} \equiv\left(y_{t}^{g a p}, \widehat{q}_{t}^{u}, \widehat{F}_{t}, \widehat{K}_{t}\right)
$$

defined as $\widehat{q}_{t}^{u} \equiv \log q_{t}^{u} / \bar{\xi}^{d}, \widehat{F}_{t} \equiv \log F_{t} / \underline{K}^{*}, \widehat{K}_{t} \equiv \log K_{t} / \underline{K}^{*}$, each evolve (to a first-order approximation) according to linear dynamics of the form

$$
w_{t}=E_{0} w_{t}+\sigma \sum_{k} \sum_{j=0}^{t-1} w_{j, k} e_{k, t-j}
$$

where $E_{0} w_{t} \sim O(\sigma)$.

Our assumption that the log deviations evolve in this way implies that (to a first-order approximation) the variables $x_{t+1}$ have surprise components

$$
\widetilde{x}_{t+1} \equiv x_{t+1}-E_{t} x_{t+1}=\sigma \sum_{k} x_{0, k} e_{k, t+1}
$$

for certain time-invariant coefficients $x_{0, k}$. We can then approximate the expectation terms in the structural equations using the approximation

$$
E_{t}\left[m_{t+1} x_{t+1}\right]=E_{t}\left[x_{t+1}\right]+E_{t}\left[\widetilde{m}_{t+1} \widetilde{x}_{t+1}\right]+O\left(\sigma^{2}\right)
$$


where

$$
\log \widetilde{m}_{t+1}=\theta^{-1} \zeta_{t}^{\prime} \widetilde{x}_{t+1}-\log E_{t}\left[\exp \left(\theta^{-1} \zeta_{t}^{\prime} \widetilde{x}_{t+1}\right)\right]
$$

is an approximation to (15) obtained using this linear approximation to the surprise component of $x_{t+1}$. Using properties of normal and log-normal random variables (see for example Yang (2008)), we can evaluate the expression

$$
E_{t}\left[\widetilde{m}_{t+1} \widetilde{x}_{t+1}\right]=M \zeta_{t},
$$

where

$$
M \equiv \frac{1}{\theta} E_{t}\left[\widetilde{x}_{t+1} \widetilde{x}_{t+1}^{\prime}\right]=\frac{1}{\theta} \sum_{k} x_{0, k} x_{0, k}^{\prime} .
$$

This allows us to write the forward-looking structural equations (30) and (53) to a first-order approximation as

$$
\begin{aligned}
\left(\widehat{q}_{t}^{u}-\widehat{q}_{t}^{u *}\right) & =\beta(1-\delta) E_{t}\left[\widehat{q}_{t+1}^{u}-\widehat{q}_{t+1}^{u *}\right]+(1-\beta(1-\delta)) s^{d}+\beta(1-\delta) M_{3} \zeta_{t} \\
\widehat{Z}_{t} & =(1-\alpha \beta)\left[\underline{z}+z_{y} y_{t}^{g a p}+z_{q}\left(\widehat{q}_{t}^{u}-\widehat{q}_{t}^{u *}\right)+z_{\xi}^{\prime} \xi_{t}\right]+\alpha \beta D(1) E_{t} \widehat{Z}_{t+1}+\alpha \beta \widetilde{M} \zeta_{t},
\end{aligned}
$$

where

$$
\widetilde{M} \equiv\left[\begin{array}{l}
M_{1} \\
M_{2}
\end{array}\right]
$$

we use the notation $M_{i}$ for the $i$-th row of $M$, and $\left(\underline{z}, z_{y}, z_{q}, z_{\xi}\right)$ are the coefficients of a loglinear approximation to the function $\log z\left(Y_{t}, q_{t}^{u} ; \xi_{t}\right)$ at the optimal steady state. This yields a system of linear relations with constant coefficients linking the endogenous variables $w_{t}$, the exogenous disturbances $\xi_{t}$, and the belief distortions $\zeta_{t}$. These equations generalize the loglinearized structural equations of the RE model, discussed in section 4, by adding the linear terms in $\zeta_{t}$. Our initial precommitment (62) can also be approximated by a linear restriction, using the approximation ${ }^{18}$

$$
\alpha \Gamma_{-1}^{\prime} \Phi\left(Z_{0}\right)+(1-\delta) \Psi_{-1} q_{0}^{u}=\underline{K}^{*} \Gamma_{-1}^{\prime} \widehat{Z}_{0}+(1-\delta) \underline{\xi}^{d} \Psi_{-1} \widehat{q}_{0}^{u}+O\left(\sigma^{3}\right) .
$$

We shall further restrict the class $\mathcal{Z}$ of belief distortions that we consider to ones of the form

$$
\zeta_{t}=E_{0} \zeta_{t}+\sigma \sum_{k} \sum_{j=0}^{t-1} \zeta_{j, k} e_{k, t-j}+O\left(\sigma^{2}\right)
$$

where $E_{0} \zeta_{t} \sim O(\sigma)$, so that the linearized structural equations (86) and (87) continue to admit solutions of the linear form (80) to a first-order approximation, just as in the RE case, when policy is specified by a linear target criterion. Note that while assumption (89) requires that belief distortions be small (of order $O(\sigma)$ ), they still modify the form of the structural equations (86) and (87) to first order. In this respect, the belief distortions allowed here are not required to be as small as those assumed in Adam and Woodford (2012), which affected the structural equations only to second order.

Appendix A.5 shows that the linearized structural equations (87) imply a generalized Phillips curve of the form

$$
\pi_{t}=\kappa_{y} y_{t}^{g a p}+\kappa_{q}\left(\widehat{q}_{t}^{u}-\widehat{q}_{t}^{u *}\right)+u_{t}+\beta E_{t} \pi_{t+1}+\kappa_{\zeta}^{\prime} \zeta_{t},
$$

where the coefficients $\kappa$ are defined in appendix and satisfy $\kappa_{y}>0, \kappa_{q}>0$. The disturbance $u_{t}$ remains the one defined in equation (77). This equation generalizes the RE Phillips curve (75) in two respects: mispricing of housing $\left(\widehat{q}_{t}^{u} \neq \widehat{q}_{t}^{u *}\right)$ has a cost-push effect, and belief distortions affecting subjective expectation of inflation shift the Phillips curve as well.

\footnotetext{
${ }^{18}$ The approximation (88) is accurate to second order because of our assumption that the multipliers $\Gamma_{-1}$ and $\Psi_{-1}$ are of order $O(\sigma)$.
} 


\section{Upper-Bound Dynamics Allowing for Belief Distor- tions}

We shall now formulate the upper bound problem, i.e., the problem on the right-hand side of inequality (7), for the nonlinear New Keynesian model with a housing market and distorted private sector expectations, and characterize its solution.

We first characterize the policymaker's best response problem (the problem in equation (6)) to an arbitrary belief distortion process $\left\{\zeta_{t}\right\}_{t=0}^{\infty} \in \mathcal{Z}$. This problem can be written as a Lagrangian problem of the form

$$
\begin{aligned}
& \max _{\left\{Y_{t}, Z_{t}, \Delta_{t}, q_{t}^{u}\right\}_{t=0}^{\infty}} E_{0} \sum_{t=0}^{\infty} \beta^{t} {\left[\begin{array}{l}
U\left(Y_{t}, \Delta_{t}, q_{t}^{u} ; \xi_{t}\right)+\theta \beta m_{t+1} \log m_{t+1} \\
+\gamma_{t}\left[\tilde{h}\left(\Delta_{t-1}, K_{t} / F_{t}\right)-\Delta_{t}\right] \\
+\Gamma_{t}^{\prime}\left[z\left(Y_{t}, q_{t}^{u}, \xi_{t}\right)+\alpha \beta m_{t+1} \Phi\left(Z_{t+1}\right)-Z_{t}\right] \\
+\Psi_{t}\left[\xi_{t}^{d}+\beta(1-\delta) m_{t+1} q_{t+1}^{u}-q_{t}^{u}\right]
\end{array}\right] } \\
&+\alpha \Gamma_{-1}^{\prime} \Phi\left(Z_{0}\right)+(1-\delta) \Psi_{-1} q_{0}^{u},
\end{aligned}
$$

where $\gamma_{t}, \Gamma_{t}$ and $\Psi_{t}$ are the Lagrange multipliers associated with the same three constraints as in sections 4.1 and 4.3, and the initial precommitment (62) has again been added to allow for a time-invariant solution. The initial precommitment specifies a value for a linear combination $\alpha \widetilde{\Gamma}_{-1}^{\prime} \Phi\left(Z_{0}\right)+(1-\delta) q_{0}^{u}$, and $\Psi_{-1}$ is the Lagrange multiplier associated with this constraint. We further use the notation $\Gamma_{-1} \equiv \Psi_{-1} \widetilde{\Gamma}_{-1}$ in the Lagrangian (91) so that our first-order conditions have a time-invariant form. In evaluating the Lagrangian (91), we substitute the process (15) for $\left\{m_{t+1}\right\}_{t=0}^{\infty}$ implied by the chosen process for $\left\{x_{t+1}\right\}_{t=0}^{\infty}$ and the process $\left\{\zeta_{t}\right\}_{t=0}^{\infty}$.

\subsection{Local Approximation of the Best-Reponse Problem}

We are interested in a local approximation of the best-response dynamics of first order in the expansion parameter $\sigma$, under the assumptions stated in section 5. The terms in the Lagrangian (91) involving belief distortions are of the form

$$
\theta E_{t}\left[m_{t+1} \log m_{t+1}\right]+\Xi_{t}^{\prime} E_{t}\left[m_{t+1} x_{t+1}\right]
$$

where

$$
\Xi_{t} \equiv\left[\begin{array}{c}
\alpha \underline{K}^{*} \Gamma_{t} \\
(1-\delta) \underline{\bar{\xi}}^{d} \Psi_{t}
\end{array}\right] .
$$

We compute a linear approximation to the best response dynamics in which the variables $w_{t+1}$ are assumed to have a solution of the form (80) for some time-invariant coefficients $w_{j, k}$.

Again using (81) we can approximate the terms in equation (92) using (82) and

$$
E_{t}\left[m_{t+1} \log m_{t+1}\right]=E_{t}\left[\widetilde{m}_{t+1} \log \widetilde{m}_{t+1}\right]+O\left(\sigma^{2}\right),
$$

where $\widetilde{x}_{t+1}$ is defined in equation (81) and $\log \widetilde{m}_{t+1}$ given in equation (83). The properties of normal and log-normal random variables, mentioned earlier, allow us to write (84) and

$$
E_{t}\left[\widetilde{m}_{t+1} \log \widetilde{m}_{t+1}\right]=\frac{1}{2 \theta} \zeta_{t}^{\prime} M \zeta_{t}
$$

where $M$ is defined in (85). Hence the terms in the Lagrangian involving the belief distortions can be approximated to second order as

$$
\theta E_{t}\left[m_{t+1} \log m_{t+1}\right]+\Xi_{t}^{\prime} E_{t}\left[m_{t+1} x_{t+1}\right]=E_{t}\left[\Xi_{t}^{\prime} x_{t+1}\right]+\frac{1}{2} \zeta_{t}^{\prime} M \zeta_{t}+\Xi_{t}^{\prime} M \zeta_{t}+O\left(\sigma^{3}\right)
$$


We show in appendix A.6 how to compute second-order approximations to the other terms in the Lagrangian (91), using a second-order Taylor expansion around the constant values of the variables associated with the optimal steady state. This allows us to write

$$
\begin{aligned}
& \sum_{t=0}^{\infty} \beta^{t}\left[U\left(Y_{t}, \Delta_{t}, q_{t}^{u} ; \xi_{t}\right)+\gamma_{t}\left(\tilde{h}\left(\Delta_{t-1}, K_{t} / F_{t}\right)-\Delta_{t}\right)\right] \\
& \quad=-\sum_{t=0}^{\infty} \beta^{t}\left[\Lambda_{\pi} \pi_{t}^{2}+\Lambda_{y}\left(y_{t}^{g a p}\right)^{2}+\Lambda_{q}\left(\widehat{q}_{t}^{u}-\widehat{q}_{t}^{u *}\right)^{2}\right]+t . i . p .+O\left(\sigma^{3}\right),
\end{aligned}
$$

where $\Lambda_{\pi}>0, \Lambda_{y}>0, \Lambda_{q}>0$, and t.i.p. collects terms that are independent of policy. ${ }^{19}$

The terms in the Lagrangian (91) that still need to be approximated are equal to

$$
\Gamma_{t}^{\prime}\left(z\left(Y_{t}, q_{t}^{u}, \xi_{t}\right)+\alpha \beta E_{t}\left[\Phi\left(Z_{t+1}\right)\right]-Z_{t}\right)+\Psi_{t}\left(\xi_{t}^{d}+\beta(1-\delta) E_{t}\left[q_{t+1}^{u}\right]-q_{t}^{u}\right)
$$

Note that we include here the terms of the form $E_{t}\left[\Xi_{t}^{\prime} x_{t+1}\right]$ from the right hand side of equation (93). Because the multipliers $\Gamma_{t}$ and $\Psi_{t}$ are of order $O(\sigma)$, approximation of the terms (95) to second order requires only a first order approximation to the constraints, i.e., the structural equations. These have been approximated to first order in section 5, see equations (86) and (87). A quadratic approximation to (95) is thus given by

$$
\begin{aligned}
& \underline{K}^{*} \Gamma_{t}^{\prime}\left[(1-\alpha \beta)\left(\underline{z}+z_{y} y_{t}^{g a p}+z_{q}\left(\widehat{q}_{t}^{u}-\widehat{q}_{t}^{u *}\right)+z_{\xi}^{\prime} \xi_{t}\right)+\alpha \beta D(1) E_{t} \widehat{Z}_{t+1}-\widehat{Z}_{t}\right] \\
& +\underline{\bar{\xi}}^{d} \Psi_{t}\left[(1-\beta(1-\delta)) s^{d}+\beta(1-\delta) E_{t}\left[\widehat{q}_{t+1}^{u}-\widehat{q}_{t+1}^{u *}\right]-\left(\widehat{q}_{t}^{u}-\widehat{q}_{t}^{u *}\right)\right] .
\end{aligned}
$$

We can similarly quadratically approximate the initial precommitment using (88).

Thus we obtain an approximation to the Lagrangian (91) that is quadratic in the policymaker's decision variables of the form

$$
\begin{aligned}
E_{0} \sum_{t=0}^{\infty} \beta^{t} & \left\{-\frac{1}{2}\left(\Lambda_{\pi} \pi_{t}^{2}+\Lambda_{y}\left(y_{t}^{g a p}\right)^{2}+\Lambda_{q}\left(\widehat{q}_{t}^{u}-\widehat{q}_{t}^{u *}\right)^{2}\right)\right. \\
& +\underline{K} \Gamma_{t}^{\prime}\left[\begin{array}{l}
\left.(1-\alpha \beta)\left(\underline{z}+z_{y} y_{t}^{g a p}+z_{q}\left(\widehat{q}_{t}^{u}-\widehat{q}_{t}^{u *}\right)+z_{\xi}^{\prime} \xi_{t}\right)\right] \\
+\alpha \beta(1) E_{t} \widehat{Z}_{t+1}-\widehat{Z}_{t}
\end{array}\right] \\
& +\underline{\xi}^{d} \Psi_{t}\left[(1-\beta(1-\delta)) s^{d}+\beta(1-\delta) E_{t}\left[\widehat{q}_{t+1}^{u}-\widehat{q}_{t+1}^{u *}\right]-\left(\widehat{q}_{t}^{u}-\widehat{q}_{t}^{u *}\right)\right] \\
& +\alpha \underline{K} \Gamma_{-1}^{\prime} D(1) \widehat{Z}_{0}+(1-\delta) \underline{\xi}^{d} \Psi_{-1} \widehat{q}_{0}^{u} \\
& \left.+\frac{1}{2} \beta \zeta_{t}^{\prime} M \zeta_{t}+\beta \Xi_{t}^{\prime} M \zeta_{t}\right\}
\end{aligned}
$$

neglecting terms independent of policy and terms of order $O\left(\sigma^{3}\right)$.

We further specialize our analysis to the case of belief distortions with the property that $\zeta_{2, t}=-\zeta_{1, t}$ at all times. This restriction on the class of contemplated belief distortions can only raise the value of the upper bound computed as the right-hand side of equation (7), so that our computed upper bound must also be an upper bound for our original problem. We show below that even this potentially looser upper bound is in fact achievable through an appropriate policy commitment; hence our restriction on the class of contemplated belief distortions is not a binding constraint.

Under this assumption, we can also show, as in the rational expectations analysis in section 4 , that in the case of an initial precommitment in which $\Gamma_{2,-1}=-\Gamma_{1,-1}$, the best response

\footnotetext{
${ }^{19}$ Note that in addition to a Taylor expansion of the objective, we have used a second-order approximation to equation (45) to substitute variations in inflation $\pi_{t}$ for variations in $K_{t} / F_{t}$. Explicit expressions for the coefficients $\Lambda_{\pi}, \Lambda_{y}$ and $\Lambda_{q}$ are provided in appendix A.6.
} 
dynamics will involve multipliers for which $\Gamma_{2, t}=-\Gamma_{1, t}$ for all $t \geq 0$. We accordingly assume this restriction as well in what follows. In this case, only two linear combinations of the vector $\widetilde{x}_{t+1}$ matter for our calculations and we can write

$$
\begin{aligned}
\Xi_{t}^{\prime} \widetilde{x}_{t+1} & =-\varphi_{t}\left(\pi_{t+1}-E_{t} \pi_{t+1}\right)-(1-\delta) \psi_{t}\left(\widehat{q}_{t+1}^{u}-E_{t} \widehat{q}_{t+1}^{u}\right) \\
\zeta_{t}^{\prime} \widetilde{x}_{t+1} & =\zeta_{t}^{\pi}\left(\pi_{t+1}-E_{t} \pi_{t+1}\right)+\zeta_{t}^{q}\left(\widehat{q}_{t+1}^{u}-E_{t} \widehat{q}_{t+1}^{u}\right),
\end{aligned}
$$

where we introduce a new parameterization of the Lagrange multipliers for the policymaker's problem,

$$
\varphi_{t} \equiv \underline{K}^{*} \frac{\alpha}{1-\alpha}(1+\omega \eta) \Gamma_{1, t}, \quad \psi_{t} \equiv-\underline{\bar{\xi}}^{d} \Psi_{t}
$$

and a new parameterization of belief distortions

$$
\widehat{\zeta}_{t} \equiv\left(\zeta_{t}^{\pi}, \zeta_{t}^{q}\right)^{\prime}
$$

where

$$
\zeta_{t}^{\pi} \equiv-\underline{K}^{*}\left(\frac{1+\omega \eta}{1-\alpha}\right) \zeta_{1, t}, \quad \zeta_{t}^{q} \equiv \underline{\bar{\xi}}^{d} \zeta_{3, t}
$$

We can then rewrite

$$
\begin{aligned}
\zeta_{t}^{\prime} M \zeta_{t} & =\theta^{-1} \widehat{\zeta}_{t}^{\prime} V \widehat{\zeta}_{t} \\
\Xi_{t}^{\prime} M \zeta_{t} & =-\theta^{-1}\left(\varphi_{t},(1-\delta) \psi_{t}\right) V \widehat{\zeta}_{t}
\end{aligned}
$$

where

$$
V \equiv E_{t}\left[\left(\pi_{t+1}-E_{t} \pi_{t+1}, \widehat{q}_{t+1}^{u}-E_{t} \widehat{q}_{t+1}^{u}\right) \cdot\left(\pi_{t+1}-E_{t} \pi_{t+1}, \widehat{q}_{t+1}^{u}-E_{t} \widehat{q}_{t+1}^{u}\right)^{\prime}\right]
$$

is a covariance matrix for the surprise components of $\pi_{t+1}$ and $\widehat{q}_{t+1}^{u}$. This allow us to write the Lagrangian (96) entirely in terms of the evolution of inflation, the output gap and housing prices.

\subsection{A Modified Linear-Quadratic Approximate Problem}

Using the results from the previous section, we rewrite the Lagrangian (96) in the form

$$
\begin{aligned}
& E_{0} \sum_{t=0}^{\infty} \beta^{t}\{-\frac{1}{2}\left(\Lambda_{\pi} \pi_{t}^{2}+\Lambda_{y}\left(y_{t}^{g a p}\right)^{2}+\Lambda_{q}\left(\widehat{q}_{t}^{u}-\widehat{q}_{t}^{u *}\right)^{2}\right)+\frac{\beta}{2 \theta} \widehat{\zeta}_{t}^{\prime} V \widehat{\zeta}_{t} \\
&+\varphi_{t}\left[\begin{array}{l}
\pi_{t}-\kappa_{y} y_{t}^{g a p}-\kappa_{q}\left(\widehat{q}_{t}^{u}-\widehat{q}_{t}^{u *}\right)-u_{t}-\beta E_{t} \pi_{t+1} \\
-\beta \theta^{-1} V_{1} \widehat{\zeta}_{t}
\end{array}\right] \\
&+\psi_{t}\left[\begin{array}{l}
\left(\widehat{q}_{t}^{u}-\widehat{q}_{t}^{u *}\right)-(1-\beta(1-\delta)) s^{d} \\
-\beta(1-\delta)\left(E_{t}\left[\widehat{q}_{t+1}^{u}-\widehat{q}_{t+1}^{u *}\right]+\theta^{-1} V_{2} \widehat{\zeta}_{t}\right)
\end{array}\right] \\
&\left.-\varphi_{-1} \pi_{0}-(1-\delta) \psi_{-1}\left(\widehat{q}_{0}^{u}-\widehat{q}_{0}^{u *}\right)\right\}
\end{aligned}
$$

where $V_{i}$ is the $i$-th row of the covariance matrix of surprises $V$ defined in (98).

The approximate Lagrangian (99) is in fact the Lagrangian for a problem of choosing evolutions for the endogenous variables $\left\{\pi_{t}, y_{t}^{g a p}, \widehat{q}_{t}^{u}\right\}_{t=0}^{\infty}$ so as to minimize a quadratic loss function

$$
E_{0} \sum_{t=0}^{\infty} \frac{\beta^{t}}{2}\left[\Lambda_{\pi} \pi_{t}^{2}+\Lambda_{y}\left(y_{t}^{g a p}\right)^{2}+\Lambda_{q}\left(\widehat{q}_{t}^{u}-\widehat{q}_{t}^{u *}\right)^{2}-\frac{\beta}{\theta} \widehat{\zeta}_{t}^{\prime} V \widehat{\zeta}_{t}\right]
$$


subject to the constraints

$$
\begin{gathered}
\pi_{t}=\beta E_{t} \pi_{t+1}+\kappa_{y} y_{t}^{g a p}+\kappa_{q}\left(\widehat{q}_{t}^{u}-\widehat{q}_{t}^{u *}\right)+u_{t} \\
+\beta \theta^{-1} V_{1} \widehat{\zeta}_{t} \\
\left(\widehat{q}_{t}^{u}-\widehat{q}_{t}^{u *}\right)=\beta(1-\delta) E_{t}\left[\widehat{q}_{t+1}^{u}-\widehat{q}_{t+1}^{u *}\right]+(1-\beta(1-\delta)) s^{d} \\
+\beta(1-\delta) \theta^{-1} V_{2} \widehat{\zeta}_{t}
\end{gathered}
$$

for all $t \geq 0$, and an initial precommitment of the form

$$
\frac{\varphi_{-1}}{\psi_{-1}} \pi_{0}+(1-\delta)\left(\widehat{q}_{0}^{u}-\widehat{q}_{0}^{u *}\right)=G_{0}
$$

where the coefficients $\varphi_{-1} / \psi_{-1}$ and $G_{0}$ are given. Here the constraints (101) and (102) represent the first-order approximations to the structural equations derived in section 5 , but with the belief distortion terms now written in terms of the smaller vector $\widehat{\zeta}_{t}$. The first two terms in the loss function (100) would also be present under the assumption of rational expectations, but the third term would not be needed (as it would be independent of policy), and the fourth term would not be present (as this reflects the penalty for large belief distortions).

The problem of minimizing the loss function (100) subject to the constraints (101) and (102) is more complicated than the linear-quadratic problem that would approximately characterize an optimal policy commitment in the case of rational expectations. First, the constraints (101) and (102), while containing only terms of order $O(\sigma)$, are no longer linear in the responses of the endogenous variables to shocks, because of the belief distortion terms (recall that $V_{1}$ and $V_{2}$ themselves depend on those responses). Second, the loss function remains a quadratic function of the responses of the endogenous variables, but it is no longer a simple sum of squared terms with positive weights, but now includes a final term that is concave rather than convex as a function of the responses of the endogenous variables.

Note that we restrict the policies that we consider to be ones that result in equilibrium dynamics, to a first order of approximation, in which the evolution of the endogenous variables is of the form (80). Thus in our approximate characterization of robustly optimal policy, the effects of the underlying disturbances $e_{k t}$ on endogenous variables such as inflation and output are linear, as in standard characterizations of optimal policy under commitment in the case of rational expectations. We can thus compare the robustly optimal dynamics that we obtain to the optimal dynamics under rational expectations by comparing the linear impulse response functions to different types of exogenous disturbances.

Because of this, we can express our endogenous variables as sums of forecastable and unforecastable components,

$$
\begin{aligned}
& \pi_{t+1}=E_{t} \pi_{t+1}+\sum_{k} \pi_{0, k} e_{k, t+1} \\
& y_{t+1}^{g a p}=E_{t} y_{t+1}^{g a p}+\sum_{k} y_{0, k} e_{k, t+1} \\
& \widehat{q}_{t+1}^{u}=E_{t} \widehat{q}_{t+1}^{u}+\sum_{k} q_{0, k} e_{k, t+1},
\end{aligned}
$$

for each $t \geq 0$. Substituting these expressions into the Lagrangian for the problem of minimizing (100) subject to the constraints (101)-(102), we find that the Lagrangian for the policymaker's best response problem can be written as the sum $L^{e}+L^{u}$, where $L^{e}$ depends only on $\left(\pi_{0}, y_{0}^{g a p}, \widehat{q}_{0}^{u}\right)$ and the predictable components $\left\{E_{t} \pi_{t+1}, E_{t} y_{t+1}^{g a p}, E_{t} \widehat{q}_{t+1}^{u}\right\}_{t=0}^{\infty}$, taking as given the evolution of the belief distortions and the Lagrange multipliers, and $L^{u}$ depends only on the coefficients $\left(\pi_{0, k}, y_{0, k}, q_{0, k}\right)$ describing the surprise components of each of the variables. 
We further observe that $L^{e}$ is a convex quadratic function of the predictable components, and accordingly has an interior minimum corresponding to the unique solution to a system of linear difference equations. ${ }^{20}$ The function $L^{u}$ is a quadratic function of the form

$$
L^{u}=E_{0} \sum_{t=0}^{\infty} \beta^{t} \sum_{k}\left[a_{t, k}^{\prime}\left(\begin{array}{c}
\pi_{0, k} \\
q_{0, k}
\end{array}\right)+\left(\pi_{0, k}, q_{0, k}\right) H\left(\begin{array}{c}
\pi_{0, k} \\
q_{0, k}
\end{array}\right)+b_{t} y_{0, k}+\frac{\Lambda_{y}}{2}\left(y_{0, k}\right)^{2}\right],
$$

where the coefficients $\left(a_{t}, b_{t}, H\right)$ are defined in appendix A.7 and where we ignored terms that are independent of policy. This is a convex function of the response coefficients if and only if the $2 \times 2$ matrix $H$ is positive semi-definite, i.e., if and only if

$$
H_{11} \geq 0 \text { and } \operatorname{det}(H) \geq 0 \text {. }
$$

As discussed in appendix A.7, conditions (106) are necessarily satisfied in the case of any large enough value for $\theta / \sigma^{2}{ }^{21}$ In this case, the response coefficients that minimize the Lagrangian are given by a system of linear equations, the coefficients of which depend on the paths of the belief distortions and the Lagrange multipliers.

The first order conditions for the minimization of $L^{e}+L^{u}$, together with the linearized structural equations (101)-(102), provide a system of equations to solve for the dynamics of the endogenous variables and the Lagrange multipliers. Assuming that this solution implies paths for the multipliers that satisfy condition (106), we have obtained a solution to the problem of minimizing the Lagrangian, given the belief distortions $\left\{\zeta_{t}^{\pi}, \zeta_{t}^{q}\right\}_{t=0}^{\infty}$.

\subsection{Worst-Case Belief Distortions}

We turn next to the problem of choosing the worst-case belief distortions in the Lagrangian upper-bound problem (8). We wish to consider the effect of variations in the belief distortions $\widehat{\zeta}=\left(\widehat{\zeta}_{0}, \widehat{\zeta}_{1}, \ldots\right)$ on the minimized value of the Lagrangian (99) for the policymaker's bestresponse problem. Let $x(\widehat{\zeta})$ denote the policymaker's best response to any belief distortions $\hat{\zeta}$, where $x=\left(x_{0}, x_{1}, \ldots\right)$ and $x_{t}=\left(\pi_{t}, y_{t}^{g a p}, \widehat{q}_{t}^{u}, \varphi_{t}, \psi_{t}\right)$. Using the notation $\Lambda(\widehat{\zeta}, x)$ for the Lagrangian (99), we wish to find the belief distortions $\hat{\zeta}$ that solve the problem

$$
\max _{\widehat{\zeta}} \Lambda(\widehat{\zeta}, x(\widehat{\zeta}))
$$

We begin by finding a saddle point of the Lagrangian, namely a pair $\left(x^{*}, \widehat{\zeta}^{*}\right)$ such that $x^{*}=x\left(\widehat{\zeta}^{*}\right)$ and at the same time $\widehat{\zeta}^{*}$ is a solution to the problem

$$
\max _{\hat{\zeta}} \Lambda\left(\widehat{\zeta}, x^{*}\right)
$$

With a solution of this kind,

$$
\Lambda(\widehat{\zeta}, x(\widehat{\zeta})) \leq \Lambda\left(\widehat{\zeta}, x^{*}\right) \leq \Lambda\left(\widehat{\zeta}^{*}, x^{*}\right)
$$

for all $\widehat{\zeta}$, so that $\widehat{\zeta}^{*}$ is in fact the solution to the worst-case belief problem (107).

We note further that the first-order conditions for problem (108) are given by

$$
\frac{\partial \Lambda}{\partial \zeta}\left(\widehat{\zeta}^{*}, x^{*}\right)=0
$$

\footnotetext{
${ }^{20}$ This system is of the same form as in the case of rational expectations.

${ }^{21}$ In our numerical illustration below, we verify numerically that conditions (106) hold.
} 
together with the requirement that $x^{*}=x\left(\hat{\zeta}^{*}\right)$. We begin by looking for a solution to this system of equations.

For given $x$, the effects of the belief distortions on the Lagrangian (99) are captured by the terms

$$
\theta^{-1} E_{0} \sum_{t=0}^{\infty} \beta^{t+1}\left[\left(\varphi_{t},(1-\delta) \psi_{t}\right) V \widehat{\zeta}_{t}-\frac{1}{2} \widehat{\zeta}_{t}^{\prime} V \widehat{\zeta}_{t}\right]
$$

where $V$ is given for given $x$. The first-order condition (109) for $\widehat{\zeta}_{t}$ delivers the condition

$$
\widehat{\zeta}_{t}^{* \prime}=\left(\varphi_{t}^{*},(1-\delta) \psi_{t}^{*}\right)
$$

where $\varphi_{t}^{*}$ and $\psi_{t}^{*}$ are the Lagrange multipliers associated with the solution to the policymaker's best-response problem. This condition implies that the worst-case belief distortions distort probability beliefs to the greatest extent precisely in those periods and states of the world in which tightening the constraints implied by the forward-looking structural equations will be most uncomfortable for the policymaker.

It remains to show that a solution to these first-order conditions is actually a solution to the maximization problem (108). A second-order expansion of $\Lambda\left(\widehat{\zeta}, x^{*}\right)$ around $\widehat{\zeta}=\widehat{\zeta}^{*}$ delivers:

$$
\Lambda\left(\widehat{\zeta}, x^{*}\right)=\Lambda\left(\widehat{\zeta}^{*}, x^{*}\right)+\frac{\partial \Lambda}{\partial \widehat{\zeta}^{\prime}}\left(\widehat{\zeta}^{*}, x^{*}\right) \widehat{\zeta}+\widehat{\zeta}^{\prime} \frac{\partial^{2} \Lambda}{\partial \widehat{\zeta}^{2} \partial \widehat{\zeta}^{\prime}}\left(\widehat{\zeta}^{*}, x^{*}\right) \widehat{\zeta}+O\left(\sigma^{3}\right)
$$

At the conjectured optimum, we have $\frac{\partial \Lambda}{\partial \widehat{\zeta}}\left(\widehat{\zeta}^{*}, x^{*}\right)=0$ from equation (109). From equation (110) follows that

$$
\frac{\partial^{2} \Lambda}{\partial \widehat{\zeta} \partial \widehat{\zeta}^{\prime}}\left(\widehat{\zeta}^{*}, x^{*}\right)=-\theta^{-1} V
$$

where $V$ is defined in equation (98) and necessarily a positive semi-definite matrix. Equation (112) thus implies that condition (108) locally holds around the conjectured solution. This proves that the saddle-point distortions (111) solve the worst-case belief problem (107).

\subsection{Linearized Upper-Bound Dynamics}

We can now substitute the solution (111) for the belief distortions into the system of linear equations characterizing the best-response dynamics. We obtain a system of linear equations ${ }^{22}$

$$
\begin{aligned}
& \Lambda_{\pi} \pi_{t}-\varphi_{t}+\varphi_{t-1}+ \theta^{-1} E_{\varphi \varphi}\left(\pi_{t}-E_{t-1} \pi_{t}\right) \\
&+\theta^{-1}(1-\delta) E_{\varphi \psi}\left(\widehat{q}_{t}^{u}-E_{t-1} \widehat{q}_{t}^{u}\right)=0 \\
& \Lambda_{y} y_{t}^{g a p}+\kappa_{y} \varphi_{t}=0 \\
& \Lambda_{q}\left(\widehat{q}_{t}^{u}-\widehat{q}_{t}^{u *}\right)+\kappa_{q} \varphi_{t}-\psi_{t}+(1-\delta) \psi_{t-1} \\
&+\theta^{-1}(1-\delta) E_{\varphi \psi}\left(\pi_{t}-E_{t-1} \pi_{t}\right) \\
&+ \theta^{-1}(1-\delta)^{2} E_{\psi \psi}\left(\widehat{q}_{t}^{u}-E_{t-1} \widehat{q}_{t}^{u}\right)=0
\end{aligned}
$$

for all $t \geq 1$, and

$$
\begin{aligned}
\pi_{t}=\kappa_{y} y_{t}^{g a p}+\kappa_{q}\left(\widehat{q}_{t}^{u}-\widehat{q}_{t}^{u *}\right) & +u_{t}+\beta E_{t} \pi_{t+1} \\
& +\beta \theta^{-1} V_{1}\left(\varphi_{t},(1-\delta) \psi_{t}\right)^{\prime}
\end{aligned}
$$

\footnotetext{
${ }^{22}$ To simplify notation, we do not add the star superscripts to the variables to indicated the upper bound solution, unlike in the previous section.
} 


$$
\begin{aligned}
\left(\widehat{q}_{t}^{u}-\widehat{q}_{t}^{u *}\right)=(1-\beta(1-\delta)) s^{d} & +\beta(1-\delta) E_{t}\left[\widehat{q}_{t+1}^{u}-\widehat{q}_{t+1}^{u *}\right] \\
& +\beta(1-\delta) \theta^{-1} V_{2}\left(\varphi_{t},(1-\delta) \psi_{t}\right)^{\prime}
\end{aligned}
$$

for all $t \geq 0$. In period $t=0$, we also have the conditions

$$
\begin{gathered}
\Lambda_{\pi} \pi_{0}-\varphi_{0}+\varphi_{-1}=0 \\
\Lambda_{y} y_{0}^{g a p}+\kappa_{y} \varphi_{0}=0 \\
\Lambda_{q}\left(\widehat{q}_{0}^{u}-\widehat{q}_{0}^{u *}\right)+\kappa_{q} \varphi_{0}-\psi_{0}+(1-\delta) \psi_{-1}=0 .
\end{gathered}
$$

together with the initial precommitment (103). Here we have introduced the notation

$$
\mathcal{E} \equiv\left(\begin{array}{cc}
E_{\varphi \varphi} & E_{\varphi \psi} \\
E_{\varphi \psi} & E_{\psi \psi}
\end{array}\right) \equiv(1-\beta) E_{0} \sum_{t=0}^{\infty} \beta^{t}\left(\begin{array}{c}
\varphi_{t} \\
\psi_{t}
\end{array}\right)\left(\varphi_{t}, \psi_{t}\right) .
$$

Equations (113)-(115), together with the special forms (118)-(120) for the initial period, correspond to the first-order conditions for the policymaker's best response, after substituting solution (111) for the worst-case beliefs. Equations (116) and (117) correspond to the linearized structural equations (101) and (102), again substituting solution (111) for the belief distortions. Taking as given the time-invariant coefficients of the matrices $\mathcal{E}$ and $V$, these equations, together with the initial precommitment (103), provide a system of five linear equations per period to solve for the dynamics of the variables $\left\{\pi_{t}, y_{t}^{g a p}, \widehat{q}_{t}^{u}, \varphi_{t}, \psi_{t}\right\}_{t=0}^{\infty}$ together with the initial multiplier $\psi_{-1}$. Note that a bounded solution to these equations will necessarily be of the conditionally linear form (104). Given such a solution, we can then compute the matrices $\mathcal{E}$ and $V$ implied by the solution, using equations (98) and (121). This allows us to define a fixed-point problem to solve for matrices $\mathcal{E}$ and $V$.

The implied dynamics of the belief distortions associated with such a solution are given by equation (111). We can then evaluate the matrix $H$ appearing in equation (105), obtaining

$$
H \equiv \frac{1}{2}\left(\begin{array}{cc}
\Lambda_{\pi}+\theta^{-1} E_{\varphi \varphi} & \theta^{-1}(1-\delta) E_{\varphi \psi} \\
\theta^{-1}(1-\delta) E_{\varphi \psi} & \Lambda_{y}+\theta^{-1}(1-\delta)^{2} E_{\psi \psi}
\end{array}\right) .
$$

Note that the definition (121) implies that $\mathcal{E}$ is a positive semi-definite matrix. Since $\Lambda_{\pi}, \Lambda_{y}>0$, equation (122) implies that $H$ must be positive definite, so that the solution to our linear system corresponds to a minimum of the Lagrangian for the policymaker's best-response problem.

A pair of matrices $\mathcal{E}$ and $V$ that solve the fixed-point problem, together with the associated solution of the linear equations (113)-(120), provide a characterization of the linearized upperbound dynamics, by which we mean a log-linear approximation to the solution to the Lagrangian upper-bound problem (8). The associated dynamics of the belief distortions are given by equation (111). We observe that the implied paths of the Lagrange multipliers $\left\{\varphi_{t}, \psi_{t}\right\}_{t=0}^{\infty}$ are indeed of order $O(\sigma)$, as assumed in section 6.1 , because both the constant term $(1-\beta(1-\delta)) s^{d}$ and the disturbance terms $\left(\widehat{q}_{t}^{u *}, u_{t}\right)$ appearing in equations (113)-(120) are of order $O(\sigma)$, due to assumption (74). Because the belief distortions are proportional to the Lagrange multipliers, in accordance with equation (111), the processes $\left\{\widehat{\zeta}_{t}\right\}_{t=0}^{\infty}$ are also of order $O(\sigma)$, as also assumed in section 6.1.

Associated with this solution for the evolution of the endogenous variables are evolutions of the complete set of Lagrange multipliers $\left\{\Gamma_{t}, \gamma_{t}, \Psi_{t}\right\}_{t=0}^{\infty}$ for the nonlinear best-response problem (91). We show in appendix A.8 that these multipliers satisfy $\left(\Gamma_{t}, \gamma_{t}-\gamma^{*}, \Psi_{t}\right) \sim O(\sigma)$, as assumed in section 6.1. Hence if the system of linear equations (113)-(120) has a bounded solution implying a matrix $H$ that is positive semi-definite, we can construct paths for all variables satisfying the assumptions made in our specification of the Lagrangian upper-bound problem. 


\section{A Robustly Optimal Target Criterion for Monetary Policy}

The equations in the previous section describe the dynamics of inflation, the output gap and housing prices that solve the upper-bound problem. It remains to be determined if there exists a monetary policy commitment that can achieve this upper bound, when belief distortions are chosen as the worst-case distortions in response to this policy commitment.

We begin by noting that conditions (113) and (114) require that under the upper-bound dynamics the joint evolution of inflation, output gap and house prices necessarily satisfy

$$
\begin{aligned}
& \pi_{t}+\frac{\Lambda_{y}}{\Lambda_{\pi} \kappa_{y}}\left(y_{t}^{g a p}-y_{t-1}^{g a p}\right) \\
& +\frac{\theta^{-1}}{\Lambda_{\pi}} E_{\varphi \varphi}\left(\pi_{t}-E_{t-1} \pi_{t}\right)+\frac{\theta^{-1}}{\Lambda_{\pi}}(1-\delta) E_{\varphi \psi}\left(\widehat{q}_{t}^{u}-E_{t-1} \widehat{q}_{t}^{u}\right)=0
\end{aligned}
$$

for all $t \geq 1$. Equation (123) is a necessary condition for achieving the upper bound and we can propose it as a target criterion for monetary policy, i.e., the central bank adjusts its policy instrument as necessary in order to ensure that condition (123) is satisfied in each period $t \geq 1 .{ }^{23}$ The coefficients $E_{\varphi \varphi}$ and $E_{\varphi \psi}$ in the target criterion should now be interpreted as specific numbers, corresponding to the values in the upper-bound dynamics, so that the target criterion establishes a linear relationship among the endogenous variables (as does the initial precommitment).

Analytically showing that the targeting rule (123) implements the upper-bound dynamics as a worst-case outcome turns out to be difficult. Nevertheless, the next section presents a general approach that allows us to verify numerically whether this is the case. Using this approach, we verify that the target criterion (123) is an example of a robustly-optimal policy commitment for the numerical example presented in section 8 below. We found this also to be true for a number of alternative model parameterizations.

The target criterion (123) generalizes the target criterion (76), which is optimal in the absence of robustness concerns (or under an assumption of rational expectations). In fact, the rational-expectations optimal policy commitment is obtained from (123) in the limiting case in which $\sigma^{2} / \theta \rightarrow 0$, which captures a setting in which belief distortions are more costly. ${ }^{24}$ The target criterion (123) also generalizes the one for a New Keynesian model without a housing sector presented in Adam and Woodford (2012), which featured only the term involving inflation surprises in the second line of equation $(123) .{ }^{25}$

\footnotetext{
${ }^{23}$ Monetary policy in period $t=0$ is specified by the requirement that the initial precommitment be satisfied.

${ }^{24}$ Recall that he coefficients $\left(E_{\varphi \varphi}, E_{\varphi \psi}\right)$ are of order $O\left(\sigma^{2}\right)$ in our setting where steady-state distortions are of order $O(\sigma)$.

${ }^{25}$ Adam and Woodford (2012) assumed $\theta \sim O(\sigma)$. In a setting where steady-state distortions are of order $O(\sigma)$, as considered here, we have $\left(E_{\varphi \varphi}, E_{\varphi \psi}\right) \sim O\left(\sigma^{2}\right)$, so that $\theta \sim O(\sigma)$ implies that the terms in the second line of (123) are all of second-order. This shows that it is important to either allow for larger belief distortions, as we do in our present setting where $\theta \sim O\left(\sigma^{2}\right)$, or to allow for steady-state distortions of order $O(0)$, as in Adam and Woodford (2012).
} 
The coefficients in the targeting rule (123) have the following signs

$$
\begin{aligned}
\frac{\Lambda_{y}}{\Lambda_{\pi} \kappa_{y}} & >0 \\
\frac{\theta^{-1} E_{\varphi \varphi}}{\Lambda_{\pi}} & >0 \\
\operatorname{sign}\left(\frac{\theta^{-1}}{\Lambda_{\pi}}(1-\delta) E_{\varphi \psi}\right) & =\operatorname{sign}\left(E_{\varphi \psi}\right) .
\end{aligned}
$$

From equation (121) follows that - for the case where the steady state distortions are sufficiently large relative to the standard deviation of the shocks ${ }^{26}$ - the sign of $E_{\varphi \psi}$ is identical to the sign of $\varphi \cdot \psi$, where the lower bars indicate the second-best steady state value of the Lagrange multipliers. From lemmas 1 and 2 and definition (97) we then obtain for the empirically relevant case with a housing subsidy $\left(s^{d}>0\right)$ and an inefficiently low output subsidy $(1-\tau<$ $\left.1-\tau^{e f f}\left(s^{s}\right)\right)$ that

$$
E_{\varphi \psi}>0
$$

A positive coefficient on housing price surprises implies that surprise increases (decreases) in housing prices require either lower (higher) inflation or a lower (higher) output gap, compared to a situation where policy is not concerned about deviations from rational expectations. It is in this sense that robustly optimal policy requires "leaning against" housing prices.

Intuitively, a situation with a positive housing subsidy and an inefficiently low output subsidy is one where there is too little (non-durable) consumption and too many houses. The policymaker thus fears belief distortions that simultaneously generate additional housing supply and an additional shortfall in consumption. This can be achieved by distorting probability beliefs in a way such that they overstate the likelihood of future states in which both inflation and housing prices are high. From the New Keynesian Phillips curve (101) it follows that upwardly distorted inflation expectations $\left(\theta^{-1} V_{1} \widehat{\zeta}_{t}>0\right)$ either cause lower output or to higher current inflation, both of which are costly. Likewise, it follows from the housing price equation (102) that upwardly distorted housing price expectations $\left(\theta^{-1} V_{2} \widehat{\zeta}_{t}>0\right)$ cause higher housing prices today and thus an increased excess supply of housing. If housing price and inflation surprises are positively correlated (the matrix $V$ features a large and positive off-diagonal element), then both of these expectations can be moved in the same direction with a single distortion (one of the elements of the vector $\widehat{\zeta}_{t}$ ), which amounts to reducing the effective cost of moving both expectations upwards. The robustly optimal policy (123) causes inflation and output surprises to be more negatively correlated when $E_{\varphi \psi}>0$. This causes the off-diagonal element of $V$ to be less positive and thus makes it harder (or more costly) to distort both expectations upwards.

Finally, we note that verification of the target criterion (123) does not require a determination of the extent to which actual housing prices deviate from "fundamental" housing prices. In fact, there is no need to respond differently to housing price surprises arising from changes in fundamentals and those due to changes in expectational errors. Nor does the target criterion make reference to shocks to housing demand or housing supply, except to the extent that these shocks affect an assessment of the policy-relevant output gap.

\subsection{Verifying that the Target Criterion Implements the Upper-Bound Dynamics}

To verify that the target criterion (123) implements the upper-bound solution as a worst-case outcome, we return to the general strategy spelled out in the last two paragraphs of section

\footnotetext{
${ }^{26}$ Specifically, we need that $s^{d} / \sigma$ and $\left(1-\tau^{e f f}\left(s^{d}\right)-(1-\tau)\right) / \sigma$ are sufficiently large.
} 
2.1. We already know that the target criterion is consistent with the solution to the Lagrangian upper-bound problem (it has been derived from its first-order conditions). It remains to show that the upper-bound solution is the locally unique outcome when policy commits to the proposed target criterion, and when beliefs are given by the upper-bound beliefs. Finally, we need to show that the upper-bound distortions are in fact the worst-case distortions when policy commits to the targeting rule (123). We now turn to these latter two issues.

We start by considering the worst-case dynamics associated with the policy commitment (123), by which we mean the equilibrium dynamics resulting from the worst-case belief distortions, as defined in equation (5). The worst-case belief distortion problem can be formulated as the choice of paths for the variables $\left\{\pi_{t}, y_{t}^{g a p}, \widehat{q}_{t}^{u}, \zeta_{t}^{\pi}, \zeta_{t}^{q}\right\}_{t=0}^{\infty}$ satisfying the target criterion (123) in each period $t \geq 1$, the initial precommitment in period $t=0$, as well as the structural equations (101) and (102), so as to maximize the loss function (100).

The worst-case belief distortion problem is an infinite-dimensional optimization problem, which is not easily expressed in a recursive form. This makes it somewhat more complicated to numerically verify that the upper-bound distortions actually achieve (locally) the maximum loss in terms of the objective function (100). We deal with this issue in two steps.

In a first step, we verify condition (157) in appendix A.10. This condition insures that the target criterion (123), the initial precommitment, and the structural equations (101) and (102) imply a locally unique outcome for the variables $\left\{\pi_{t}, y_{t}^{g a p}, \widehat{q}_{t}^{u}\right\}_{t=0}^{\infty}$ for given belief distortions $\left\{\zeta_{t}^{\pi}, \zeta_{t}^{q}\right\}_{t=0}^{\infty}$ in the neighborhood of the worst case belief distortions. This insures that we have a well-defined outcome function $O(\zeta, c)$ that determines the endogenous variables for alternative belief distortion, as assumed in equation (5), and that the outcome associated with the upperbound solution is indeed the locally determinate outcome implied by the target criterion when the belief distortions are those associated with the upper-bound solution.

In a second step, we show in appendix A.9 that for a given matrix $V$, the first-order conditions to the worst-case belief distortion problem in which policy commits to the targeting rule (123) imply that the predictable part of the worst-case belief distortions $E_{t-1}\left(\zeta_{t}^{\pi}, \zeta_{t}^{q}\right)$ are identical to the predictable part of the multipliers $E_{t-1}\left(\varphi_{t},(1-\delta) \psi_{t}\right)$ solving the upper bound equations (113)-(120) for the same matrix $V$. The predictable part of the multipliers thus have a recursive solution. This insight allows us to numerically verify whether the target criterion (123) implements the upper-bound solution by considering the effects of variations in a finite number of parameters.

Specifically, we consider alternative values for $V$ in the neighborhood of the values implied by the upper-bound solution, and the associated predictable dynamics of the belief distortions. We then consider alternative response coefficients to the surprise components of belief distortions (in the neighborhood of the response coefficients assume in the upper-bound solution), such that they give rise to equilibrium dynamics consistent with the hypothesized matrix $V$. In this way, we have transformed a potentially infinite-dimensional optimization problem of choosing alternative belief distortions $\left\{\zeta_{t}^{\pi}, \zeta_{t}^{q}\right\}_{t=0}^{\infty}$ into a problem of varying a small number of parameters, in fact only four. Details of the numerical procedures are spelled out in online Appendix C.

Using this approach, we check for the numerical example presented in the next section whether alternative belief distortions in the neighborhood of the upper-bound distortions yield higher or lower losses in terms of the objective function (100). ${ }^{27}$ Figure 1 depicts the distribution of incremental welfare losses relative to the losses associated with the upper-bound distortions. The figure is obtained by considering 1000 random alternative belief distortion choices in the neighborhood of the upper-bound distortions. The figure shows that additional losses are negative, i.e., that the alternative belief distortions lead to lower losses for the policymaker than those in the upper-bound solution. The belief distortions associated with the upper-bound solution thus represent worst-case belief distortions, so that the proposed target criterion (123)

\footnotetext{
${ }^{27}$ Obviously, we first check that condition (157) is in fact satisfied.
} 


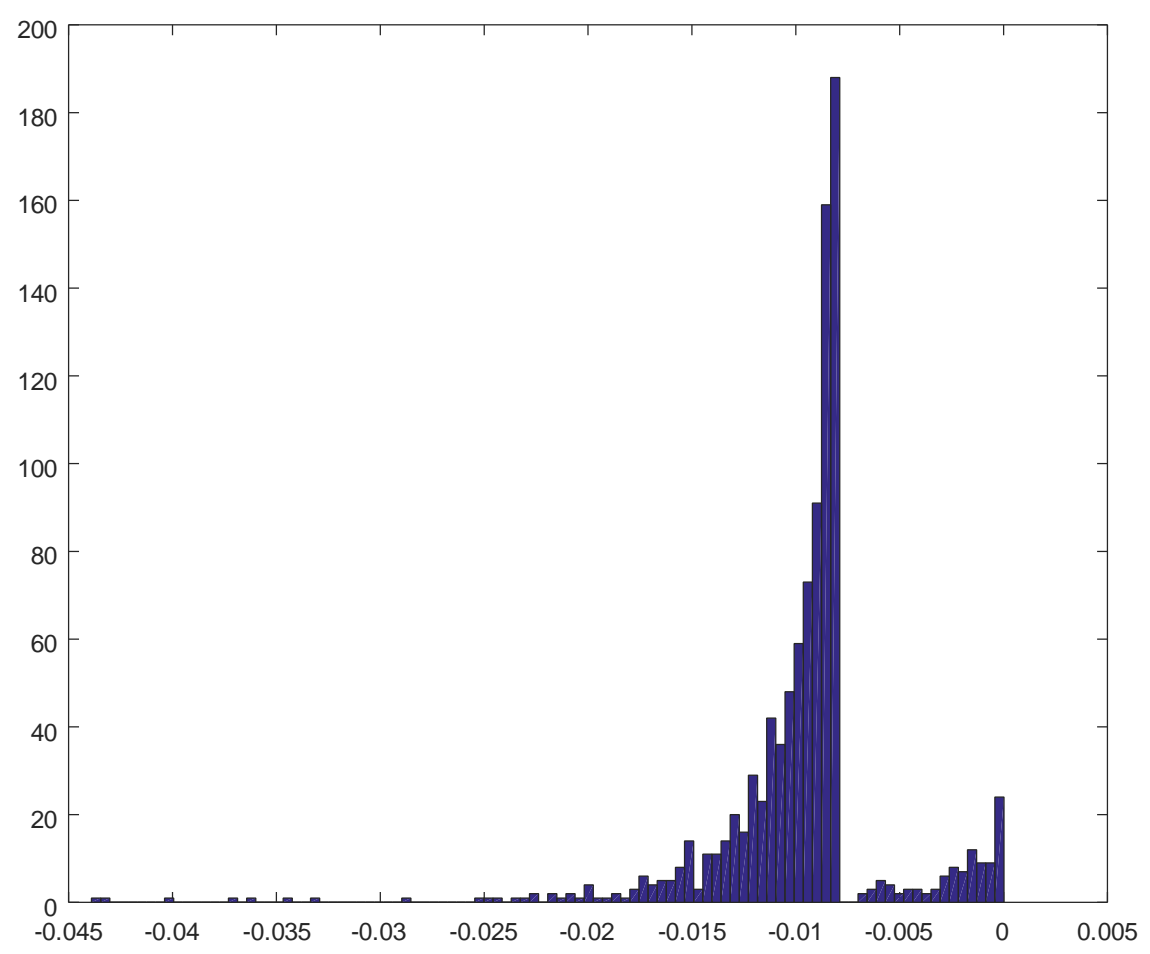

Figure 1: Distribution of incremental welfare losses associated with belief distortions in the neighborhood of the upper-bound distortions, when policy commits to the targeting rule (123). Parameteric example from section 8.

has the upper-bound solution as its worst-case outcome.

\section{A Numerical Illustration}

This section determines the upper-bound dynamics, compares them to the RE equilibrium dynamics and computes the robustly optimal targeting criterion for a numerical example. Details of the computations are reported in the online appendix of the paper.

Numerically solving for the upper-bound solution requires parameter values for $\left(\beta, \delta, \kappa_{y}, \kappa_{q}\right.$, $\Lambda_{Y} / \Lambda_{\pi}, \Lambda_{q} / \Lambda_{\pi}, s^{d}$ ), a value for the (scaled) robustness parameter $\theta^{-1} / \Lambda_{\pi}$, as well parameters characterizing the stochastic processes for $u_{t}$ and $\widehat{\bar{\xi}}_{t}^{d}$. We consider exogenous disturbances of the form

$$
\begin{aligned}
u_{t} & \equiv w+\widehat{u}_{t} \\
\widehat{q}_{t}^{u *} & =\widehat{\bar{\xi}}_{t}^{d}-s^{d},
\end{aligned}
$$

where

$$
\begin{aligned}
w & \equiv \frac{(1-\alpha)(1-\alpha \beta)}{\alpha(1+\omega \eta)} \Theta \\
\widehat{u}_{t} & \equiv \frac{(1-\alpha)(1-\alpha \beta)}{\alpha(1+\omega \eta)}\left(\widehat{\tau}_{t}-\widehat{g}_{t}\right),
\end{aligned}
$$


where $\widehat{u}_{t}$ and $\widehat{\bar{\xi}}_{t}^{d}$ are autoregressive processes of the form

$$
\begin{aligned}
& \widehat{u}_{t}=\rho_{u} \widehat{u}_{t-1}+e_{t}^{u} \\
& \widehat{\bar{\xi}}_{t}^{d}=\rho_{\xi} \widehat{\bar{\xi}}_{t-1}^{d}+e_{t}^{\xi},
\end{aligned}
$$

with $e_{t}^{u} \sim i i N\left(0,\left(\sigma_{e^{u}}\right)^{2}\right), e_{t}^{\xi} \sim i i N\left(0,\left(\sigma_{e} \xi\right)^{2}\right)$

Table 1 reports our model parameterization, which is motivated in detail in appendix A.11. The parameterization assumes the presence of a steady state housing subsidy, which will lead to an oversupply of housing in the second best steady state relative to the optimal steady state. It also assumes an output subsidy that falls $15 \%$ short of its efficient level, so that steadystate output will be below its optimal steady-state value. For the case where the robustness parameter $\theta^{-1} / \Lambda_{\pi}$ is set to zero instead of the value reported in table 1 , one obtains the optimal dynamics in the absence of robustness concerns, i.e., under rational expectations.

\begin{tabular}{lll}
\hline Discount factor & $\beta$ & 0.99 \\
Housing depreciation rate & $\delta$ & $0.03 / 4$ \\
Phillips curve coeff. on output gap & $\kappa_{y}$ & 0.024 \\
Phillips curve coeff. on house price gap & $\kappa_{q}$ & -0.0023 \\
Relative weight on output gap & $\frac{\Lambda_{Y}}{\Lambda_{\pi}}$ & 0.0031 \\
Relative weight on housing gap & $\frac{\Lambda_{q}}{\Lambda_{\pi}}$ & 0.0014 \\
Steady state housing subsidy & $s^{d}$ & $15 \%$ \\
Steady state mark-up gap & $w$ & 0.0057 \\
Mark-up shock persistence & $\rho_{u}$ & 0.9907 \\
Housing preference shock persistence & $\rho_{\xi}$ & 0.99 \\
Std. dev. mark-up shock innovation & $\sigma_{e^{u}}$ & 0.0002 \\
Std. dev. housing pref. shock innovation & $\sigma_{e^{\xi}}$ & 0.024 \\
Robustness parameters & $\frac{\theta^{-1}}{\Lambda_{\pi}}$ & 50 \\
\hline
\end{tabular}

Table 1: Parameterization (quarterly model)

Table 2 reports the average values of the output gap, the inflation rate and the housing price gap. The table reports these values for the RE solution and for the upper-bound solution. ${ }^{28}$ For the RE solution, the average values correspond to the ones in the second-best steady state. For the upper-bound solution, the average values differ from the second-best steady state due to the presence of belief distortions. In the REE, housing prices are on average $15 \%$ above their efficient value, in line with the assumed housing subsidy. The high level of housing prices causes equilibrium housing supply to be inefficiently high. Moreover, due to the suboptimally low output subsidy, the average output gap is negative, despite suboptimally high levels of housing investment. As a result, non non-durable consumption (not reported in the table) is also depressed relative to its optimal steady-state value. In the upper-bound solution, the output gap is on average less negative than in the RE equilibrium. This is due to the fact that the housing price gap is more than 5 percentage points larger than under RE. This shows that worst case beliefs distort (on average) housing prices further upwards and by doing so give rise to even higher levels of housing investment. This leads to a slightly reduced output gap. The

\footnotetext{
${ }^{28}$ The upper-bound solution is the outcome associated with a robustly optimal policy commitment and the associated worst case beliefs, while the RE solution is associated with an RE optimal policy commitment and no belief distortions.
} 
inflation rate is on average equal to zero in both solutions. Conditional inflation dynamics, however, differ notably, as we show next.

\begin{tabular}{lcc} 
& RE & Upper-bound \\
& solution & solution \\
\hline Output gap $\left(\widehat{Y}-\widehat{Y}^{*}\right)$ & $-22.3 \%$ & $-21.8 \%$ \\
Inflation $(\pi)$ & $0 \%$ & $0 \%$ \\
Housing price gap $\left(\widehat{q}^{u}-\widehat{q}^{u *}\right)$ & $15 \%$ & $20.3 \%$ \\
\hline
\end{tabular}

Table 2: Average values

Figure 2 depicts the impulse response of the output gap, the inflation rate, the price level and the housing price to a positive innovation in housing preferences $e_{t}^{\xi}$ of one standard deviation. To facilitate comparisons across equilibria, the housing price response in figure 2 is shown in terms of deviations of housing prices from their second-best steady state value. A demand shock of the considered size causes housing prices to increase in a persistent manner, with an impact effect of approximately $2.4 \%{ }^{29}$ In the REE, the inflation rate and the output gap do not respond to the housing shock, in line with the results derived in section 4.5. Under the upper-bound solution, it becomes optimal to lean against the housing price increase: inflation and the output gap both fall persistently below their steady state level. On impact, inflation is almost $0.15 \%$ and output more than $0.5 \%$ lower than in the absence of the housing demand shock. Given the relatively small size of the housing price innovation, this is a sizeable response. Stronger (weaker) robustness concerns, i.e., higher (lower) values for $\left(\theta / \Lambda_{\pi}\right)^{-1}$ ), would cause this reaction to be stronger (weaker). Under the upper bound dynamics, the price level remains permanently lower following the housing price surprise, i.e., the price level incorporates a random walk component under robustly optimal policy, while the RE optimal policy implies a stationary price level.

It is worth emphasizing that the optimal reaction to positive and negative shocks is symmetric in the upper-bound dynamics: for a negative housing demand shock, the upper-bound solution calls for a looser policy than under RE, i.e., for a persistent increase in inflation and the output gap. The online appendix of the paper also reports the impulse responses to a positive mark-up shock. Inflation then reacts identically under both policies and the output gap and the housing price gap differ along the impulse response across the two solutions only by a constant that is equal to the corresponding difference in average values reported in table 2.

Table 3 reports the coefficients for the robustly optimal targeting rule (123) that implements the upper-bound solution as a worst case equilibrium outcome. The response coefficient on the housing price surprise in this targeting rule turns out to be rather sizable. For instance, it implies that a $2.4 \%$ housing price surprise would call on impact for a $0.75 \%$ drop in the output gap when keeping inflation at its pre-shock zero value; alternatively, it would call for a $0.37 \%$ drop in (annualized) inflation when keeping the output gap at its pre-shock average value ${ }^{30}$, which is a sizable inflation response, as it amounts to approximately $15 \%$ of the housing price surprise. In the worst-case equilibrium outcome associated with a commitment to the considered robustly optimal targeting rule, the output gap and inflation both fall on impact, but each by smaller amounts, see figure 2 .

\footnotetext{
${ }^{29}$ The impact effect is exactly $2.4 \%$ in the RE solution and $2.56 \%$ in the upper bound dynamics, as belief distortions amplify the effect of the shock.

${ }^{30}$ The latter number is obtained by dividing $0.024 \cdot 0.0406$, i.e., the product of the housing price suprise and the response coefficient in the targeting rule, by 1.044, which the sum of the coefficient on inflation and the inflation surprise in the targeting rule, and by mutliplying the result by 4 to obtain annualized rates of inflation.
} 

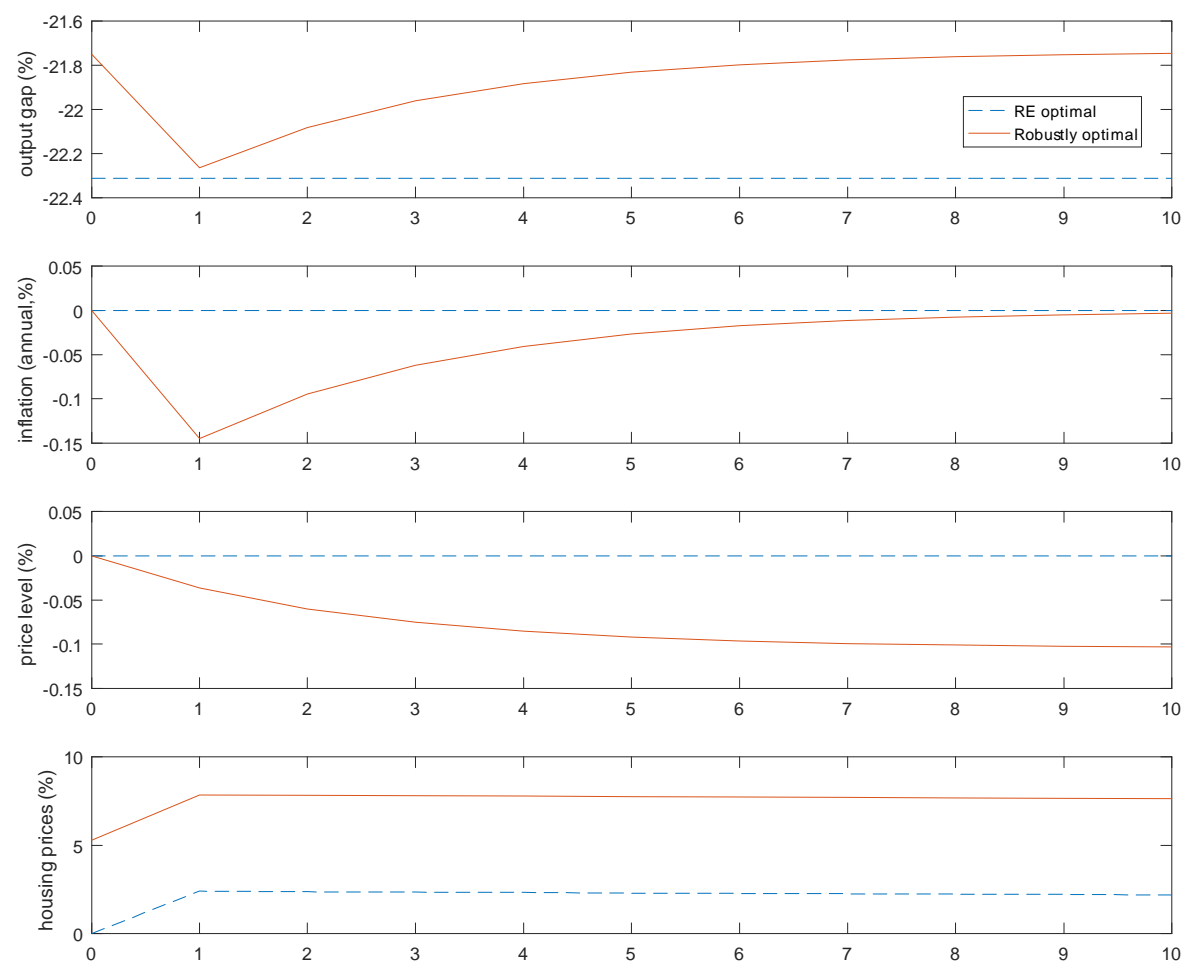

Figure 2: RE vs. robustly optimal dynamics (housing pref. shock, +1 std.dev.)

\begin{tabular}{llcc} 
Coefficient on & & RE optimal & Robustly optimal \\
\hline Change in output gap & $\frac{\Lambda_{y}}{\Lambda_{\pi} \kappa_{y}}$ & 0.1292 & 0.1292 \\
Inflation surprises & $\frac{\theta^{-1}}{\Lambda_{\pi}} E_{\varphi \varphi}^{\text {new }}$ & 0 & 0.0414 \\
Housing price surprises & $\frac{\theta^{-1}}{\Lambda_{\pi}}(1-\delta) E_{\varphi \psi}^{\text {new }}$ & 0 & 0.0406 \\
\hline
\end{tabular}

Table 3: Optimal targeting rule coefficients

\section{Conclusions}

Monetary policymakers concerned about whether private-sector expectations will necessarily coincide with those implied by their own model, that they use to understand the economy and choose their policy commitment, may find it desirable to include housing prices in the set of variables that they must track in order to verify that policy is on course, alongside the traditional "target variables" of inflation and a suitably defined measure of the output gap. This can be the case even under circumstances where an optimal policy commitment could be formulated purely in terms of a desired relationship between the paths of inflation and the output gap, if one could be confident that one's policy would result in a rational-expectations equilibrium. We have illustrated this in the context of a standard New Keynesian model extended to include a housing sector, where we find that robustly optimal policy can be characterized by a linear "target criterion," but this must involve housing price surprises in addition to the paths of inflation and the output gap. In the presence of a housing subsidy, this requires monetary policy to be tighter (less tight) following unexpected increases (decreases) in the housing price 
than in the case in which the policymaker can rely on the private sector to have the same expectations as herself.

Of course, our analysis does not pretend to provide a complete analysis of the problem of the desirable policy response to housing booms and busts. In our simple model, mis-pricing of housing due to expectational errors matters for welfare only because of its consequences for the degree to which productive resources are drawn into the housing sector; hence the dependence of our results on the degree to which there is already an inefficient over-supply of housing in the steady state, owing to housing subsidies. We believe that this is one reason why housing booms are harmful, but it probably is not the only one. Central banks' concern to "lean against" housing booms is often based on the fear that both household and bank balance sheets may be impaired in the event of a subsequent collapse of housing prices, as a result of the increased household borrowing often observed during a housing boom. Our model does not address this issue, as for simplicity we abstract both from household borrowing and from the existence of banks. The exercise must therefore be viewed more as an illustration of our proposed approach than as a complete treatment of a policy issue. It should, however, suffice to indicate that conclusions about the need to include asset prices among the target variables based on a rational-expectations analysis need not be robust to an allowance for even modest departures from rational expectations.

\section{References}

Adam, K., A. Marcet, and J. Beutel (2017): "Stock Price Booms and Expected Capital Gains," Amercian Economic Review, Vol. 107 No. 8, 2352-2408.

Adam, K., A. Marcet, and P. Kuang (2012): "House Price Booms and the Current Account," in NBER Macroeconomics Annual 2011, ed. by D. Acemoglu, and M. Woodford, pp. $77-122$.

Adam, K., A. Marcet, and J. P. Nicolini (2016): "Stock Market Volatility and Learning," Journal of Finance, 71(1), 33-82.

Adam, K., And M. Woodford (2012): "Robustly Optimal Monetary Policy in a Microfounded New-Keynesian Model," Journal of Monetary Economics, 59, 468-487.

Benigno, P., And L. Paciello (2014): "Monetary Policy, Doubts and Asset Prices," Journal of Monetary Economics, 64, 85-98.

Benigno, P., and M. Woodford (2005): "Inflation Stabilization And Welfare: The Case Of a Distorted Steady State," Journal of the European Economic Association, 3, 1185-1236.

Bernanke, B., And M. Gertler (1999): "Monetary Policy and Asset Price Volatility," in New Challenges for Monetary Policy, Federal Reserve Bank of Kansas City, Jackson Hole Conference Proceedings.

(2001): "How Should Central Bankers Respond to Asset Prices," American Economic Review Papers and Proceedings, Vol. 91, 253-257.

Caines, C., And F. Winkler (2019): "Asset Price Beliefs and Optimal Monetary Policy," Federal Reserve Board mimeo.

Calvo, G. A. (1983): "Staggered Contracts in a Utility-Maximizing Framework," Journal of Monetary Economics, 12, 383-398. 
Christiano, L., C. Ilut, R. Motto, and M. Rostagno (2010): "Monetary Policy and Stock Market Booms," in Macroeconomic Challenges: The Decade Ahead, 2010 Jackson Hole Symposium.

Galí, J. (2014): "Monetary Policy and Rational Asset Price Bubbles," American Economic Review, 104 (3), 721-52.

Giannoni, M., and M. Woodford (2017): "Optimal Target Criteria for Stabilization Policy," Journal of Monetary Economics, 168, 55-106.

Gilchrist, S., And J. V. Leahy (2002): "Monetary Policy and Asset Prices," Journal of Monetary Economics, 49, 75-97.

Hansen, L. P., and M. Marinacci (2016): "Amibuity Aversion and Model Misspecification: An Economic Perspective," Statistical Science, 31(4), 511-515.

Hansen, L. P., and T. J. Sargent (2005): "Robust Estimation and Control under Commitment," Journal of Economic Theory, 124, 258-301.

Hansen, L. P., and T. J. Sargent (2008): Robustness. Princeton University Press, Princeton, Standord University, mimeo.

Ireland, P. (2004): "Technology Schocks in the New Keynesian Model," Review of Economics and Statistics, 86, 923-936.

Maccheroni, F., M. Marinacci, and A. Rustichini (2006): "Amibuity Aversion, Robustness, and the Variational Representation of Preferences," Econometrica, 74(6), 1447-1489.

Miao, J., Z. Shen, and P. Wang (2020): "Monetary Policy and Rational Asset Price Bubbles: Comments," American Economic Review (forthcoming).

Sims, C. (2000): "Solving Linear Rational Expectations Models," Princeton University mimeo.

Svensson, L. E. O. (1999): "Inflation targeting as a monetary policy rule," Journal of Monetary Economics, 43, 607-654.

Taylor, J. B. (2007): "Housing and Monetary Policy," in Housing, Housing Finance, and Monetary Policy, Jackson Hole Symposium. Kansas City Fed.

Topel, R., and S. Rosen (1988): "Housing Investment in the United States," Journal of Political Economy, 96, 718-740.

Woodford, M. (2003): Interest and Prices. Princeton University Press, Princeton. (2010): "Robustly Optimal Monetary Policy with Near-Rational Expectations," American Economic Review, 100, 274-303.

(2011): "Optimal Monetary Stabilization Policy," in Handbook of Monetary Economics, ed. by B. M. Friedman, and M. Woodford, vol. 3B. Elsevier, Amsterdam.

YANG, M. (2008): "Normal log-normal mixture, leptokurtosis and skewness," Applied Economics Letters, 15(9), 737-742.

Yun, T. (1996): "Nominal Price Rigidity, Money Supply Endogeneity, and Business Cycles," Journal of Monetary Economics, 27, 345-370. 


\section{A Appendix}

\section{A.1 Steady State Results}

This appendix proves a number of claims made in sections 4.2 and 4.3. Using (40) and (41) on can write (64) more explicitly as

$$
\lambda \phi \frac{\bar{H}^{-\nu}}{\underline{A}^{1+\omega}} \underline{Y}^{\omega}=\frac{\eta-1}{\eta}(1-\underline{\tau}) \bar{C}^{\tilde{\sigma}^{-1}} C\left(\underline{Y}, \underline{q}^{u}, \underline{\xi}\right)^{-\tilde{\sigma}^{-1}} .
$$

Since the left-hand side is increasing and the right-hand side decreasing in $\underline{Y}$ (as $C_{Y}>0$ ), there is a unique value for $\underline{Y}$ solving this equation, as claimed.

Using the definitions of $k$ and $f$ and (124), we have

$$
\begin{aligned}
f_{Y} & =(1-\tau) \bar{C}^{\tilde{\sigma}^{-1}} C\left(Y, q^{u}, \xi\right)^{-\widetilde{\sigma}^{-1}} \\
& -\widetilde{\sigma}^{-1}(1-\tau) \bar{C}^{\tilde{\sigma}^{-1}} Y C\left(Y, q^{u}, \xi\right)^{-\widetilde{\sigma}^{-1}-1} C_{Y}\left(Y, q^{u}, \xi\right) \\
k_{Y} & =\frac{\eta}{\eta-1} \lambda \phi \frac{\bar{H}^{-\nu}}{A^{1+\omega}}(1+\omega) Y^{\omega} \\
& =(1+\omega)(1-\underline{\tau}) \bar{C}^{\tilde{\sigma}^{-1}} \underline{C}\left(\underline{Y}, \underline{q}^{u}, \underline{\xi}\right)^{-\tilde{\sigma}^{-1}},
\end{aligned}
$$

so that from $C_{Y}>0$ and $\omega>0$ we get

$$
\begin{aligned}
k_{Y}-f_{Y} & =(1-\underline{\tau}) \bar{C}^{\tilde{\sigma}^{-1}} \underline{C}\left(\underline{Y}, \underline{q}^{u}, \underline{\xi}\right)^{-\tilde{\sigma}^{-1}} \\
& \cdot\left(\omega+\tilde{\sigma}^{-1} Y C\left(Y, q^{u}, \xi\right)^{-1} C_{Y}\left(Y, q^{u}, \xi\right)\right) \\
& >0 .
\end{aligned}
$$

From (49) we get

$$
\begin{aligned}
U_{Y}\left(Y_{t}, \Delta_{t}, q_{t}^{u} ; \xi_{t}\right) & =\bar{C}_{t}^{\tilde{\sigma}^{-1}} C\left(Y_{t}, q_{t}^{u}, \xi_{t}\right)^{-\tilde{\sigma}^{-1}} C_{Y}\left(Y_{t}, q_{t}^{u}, \xi_{t}\right) \\
& -\frac{\lambda}{1+\nu}(1+\omega) \frac{\bar{H}_{t}^{-\nu}}{A_{t}^{1+\omega}} Y_{t}^{\omega} \Delta_{t} \\
& +\frac{A_{t}^{d} \bar{\xi}_{t}^{d}}{\tilde{\alpha}} \Omega\left(q_{t}^{u}, \xi_{t}\right)^{\tilde{\alpha}}\left(\frac{\tilde{\alpha}}{1-\tilde{\alpha}} \tilde{\sigma}^{-1}\right) C\left(Y_{t}, q_{t}^{u}, \xi_{t}\right)^{\frac{\tilde{\alpha}}{1-\tilde{\alpha}} \tilde{\sigma}^{-1}-1} C_{Y}\left(Y_{t}, q_{t}^{u}, \xi_{t}\right)
\end{aligned}
$$

Using (124), $1+\omega=\phi(1+\nu)$ and evaluating at the steady state we have

$$
\begin{aligned}
U_{Y}\left(\underline{Y}, 1, \underline{q}^{u} ; \underline{\xi}\right) & =\bar{C}^{\tilde{\sigma}^{-1}} C\left(\underline{Y}, \underline{q}^{u}, \underline{\xi}\right)^{-\tilde{\sigma}^{-1}} C_{Y}\left(\underline{Y}, \underline{q}^{u}, \underline{\xi}\right) \\
& -\frac{\eta-1}{\eta}(1-\underline{\tau}) \bar{C}^{\tilde{\sigma}^{-1}} C\left(\underline{Y}, \underline{q}^{u}, \underline{\xi}\right)^{-\tilde{\sigma}^{-1}} \\
& +\frac{A^{d} \bar{\xi}}{\tilde{\alpha}} \underline{\Omega}^{\tilde{\alpha}}\left(\frac{\tilde{\alpha}}{1-\tilde{\alpha}} \tilde{\tilde{\sigma}}^{-1}\right) C\left(\underline{Y}, \underline{q}^{u}, \underline{\xi}\right)^{\frac{\tilde{\alpha}}{1-\tilde{\alpha}} \tilde{\sigma}^{-1}-1} C_{Y}\left(\underline{Y}, \underline{q}^{u}, \underline{\xi}\right) .
\end{aligned}
$$

Using the fact that at the steady state

$$
C_{Y}\left(\underline{Y}, \underline{q}^{u}, \underline{\xi}\right)=\frac{1-g}{1+\underline{\Omega} \frac{\widetilde{\sigma}^{-1}}{1-\widetilde{\alpha}} C\left(\underline{Y}, \underline{q}^{u}, \underline{\xi}\right)^{\frac{\tilde{\sigma}^{-1}}{1-\widetilde{\alpha}}-1}}
$$


and (33) we have

$$
\begin{aligned}
U_{Y}\left(\underline{Y}, 1, \underline{q}^{u} ; \underline{\xi}\right)= & \bar{C}^{\tilde{\sigma}^{-1}} C\left(\underline{Y}, \underline{q}^{u}, \underline{\xi}\right)^{-\tilde{\sigma}^{-1}} C_{Y}\left(\underline{Y}, \underline{q}^{u}, \underline{\xi}\right) \\
& -\bar{C}^{\tilde{\sigma}^{-1}} C\left(\underline{Y}, \underline{q}^{u}, \underline{\xi}\right)^{-\tilde{\sigma}^{-1}} \frac{\eta-1}{\eta}(1-\underline{\tau}) \\
& +\bar{C}^{\tilde{\sigma}^{-1}} C\left(\underline{Y}, \underline{q}^{u}, \underline{\xi}\right)^{-\tilde{\sigma}^{-1}} C_{Y}\left(\underline{Y}, \underline{q}^{u}, \underline{\xi}\right) \underline{\Omega} \frac{1}{1+s^{d}}\left(\frac{\tilde{\sigma}^{-1}}{1-\tilde{\alpha}}\right) C\left(\underline{Y}, \underline{q}^{u}, \underline{\xi}\right)^{\frac{\tilde{\sigma}^{-1}}{1-\tilde{\alpha}}-1} \\
= & \bar{C}^{\tilde{\sigma}^{-1}} C\left(\underline{Y}, \underline{q}^{u}, \underline{\xi}\right)^{-\tilde{\sigma}^{-1}} \\
& \cdot\left(-\frac{\eta-1}{\eta}(1-\underline{\tau})+C_{Y}\left(\underline{Y}, \underline{q}^{u}, \underline{\xi}\right) \frac{1+s^{d}+\underline{\Omega} \frac{\tilde{\sigma}^{-1}}{1-\tilde{\alpha}} C\left(\underline{Y}, \underline{q}^{u}, \underline{\xi}\right)^{\frac{\tilde{\sigma}^{-1}}{1-\alpha}-1}}{1+s^{d}}\right) \\
= & \bar{C}^{\tilde{\sigma}^{-1}} C\left(\underline{Y}, \underline{q}^{u}, \underline{\xi}\right)^{-\tilde{\sigma}^{-1}}\left(\frac{1-g}{1+s^{d}}-\frac{\eta-1}{\eta}(1-\underline{\tau})+\frac{s^{d}}{1+s^{d}} C_{Y}\left(\underline{Y}, \underline{q}^{u}, \underline{\xi}\right)\right) .
\end{aligned}
$$

\section{A.2 Proof of Lemma 1}

The efficient output subsidy $1-\underline{\tau}^{e f f}\left(s^{d}\right)$ is the one giving rise to $U_{Y}\left(\underline{Y}, 1, \underline{q}^{u} ; \underline{\xi}\right)=0$ and is implicitly defined as

$$
\begin{aligned}
1-\underline{\tau}^{e f f}\left(s^{d}\right) & =\frac{\eta}{\eta-1}\left(\frac{1-g+s^{d} C_{Y}\left(\underline{Y}, \underline{q}^{u}, \underline{\xi}\right)}{1+s^{d}}\right) \\
& =\frac{\eta}{\eta-1}(1-g)\left(\frac{1+\frac{s^{d}}{1+\underline{\Omega} \frac{\tilde{\sigma}^{-1}}{1-\tilde{\alpha}} C\left(\underline{Y}, \underline{q}^{u}, \underline{\xi}\right)^{\frac{\tilde{\sigma}}{1-1}-1}}}{1+s^{d}}\right) .
\end{aligned}
$$

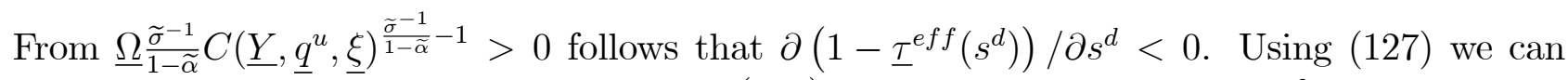
express the terms in the last parenthesis in (128), which determine the sign of $U_{Y}$ whenever $\tau$ deviates from $\underline{\tau}^{e f f}\left(s^{d}\right)$, as

$$
\begin{aligned}
& \frac{1-g}{1+s^{d}}-\frac{\eta-1}{\eta}(1-\underline{\tau})+\frac{s^{d}}{1+s^{d}} C_{Y}\left(\underline{Y}, \underline{q}^{u}, \underline{\xi}\right) \\
& =\frac{1-g}{1+s^{d}}-\frac{\eta-1}{\eta}(1-\underline{\tau})+\frac{s^{d}}{1+s^{d}} \frac{1-g}{1+\underline{\Omega} \frac{\widetilde{\sigma}^{-1}}{1-\widetilde{\alpha}} C\left(\underline{Y}, \underline{q}^{u}, \underline{\xi}\right)^{\frac{\sigma^{-1}}{1-\widetilde{\alpha}}-1}} .
\end{aligned}
$$

The derivative of the r.h.s of $(129)$ w.r.t. $(1-\underline{\tau})$ is given by

$$
-\frac{\eta-1}{\eta}-\frac{s^{d}}{1+s^{d}} \frac{1-g}{\left(1+\underline{\Omega} \frac{\widetilde{\sigma}^{-1}}{1-\widetilde{\alpha}} C\left(\underline{Y}, \underline{q}^{u}, \underline{\xi}\right)^{\frac{\tilde{\sigma}^{-1}}{1-\widetilde{\alpha}}-1}\right)^{2}} C_{Y}\left(\underline{Y}, \underline{q}^{u}, \underline{\xi}\right) \frac{\partial \underline{Y}}{\partial(1-\underline{\tau})}
$$

and is strictly negative because $C_{Y}>0$ and because (124) implies $\frac{\partial \underline{Y}}{\partial(1-\underline{\tau})}>0$. Since $U_{Y}=0$ for $\underline{\tau}=\underline{\tau}^{e f f}\left(s^{d}\right)$ this shows that $U_{Y}<0$ whenever $1-\underline{\tau}>1-\underline{\tau}^{e f f}\left(s^{d}\right)$ and $U_{Y}>0$ whenever $1-\underline{\tau}<1-\underline{\tau}\left(s^{d}\right)$, as claimed in lemma 1 . 


\section{A.3 Proof of Lemma 2}

From (49) we obtain using (33)

$$
\begin{aligned}
U_{q^{u}}\left(\underline{Y}, \underline{q}^{u}, \underline{\xi}\right) & =\bar{C}^{\tilde{\sigma}^{-1}} C\left(\underline{Y}, \underline{q}^{u}, \underline{\xi}\right)^{-\tilde{\sigma}^{-1}} C_{q^{u}}\left(\underline{Y}, \underline{q}^{u}, \underline{\xi}\right) \\
& +\underline{A}^{d} \underline{\bar{\xi}}^{d} \underline{\Omega}^{\tilde{\alpha}} \frac{\tilde{\sigma}^{-1}}{1-\tilde{\alpha}} C\left(\underline{Y}, \underline{q}^{u}, \underline{\xi}\right)^{\frac{\tilde{\alpha}}{1-\tilde{\alpha}} \tilde{\sigma}^{-1}-1} C_{q^{u}}\left(\underline{Y}, \underline{q}^{u}, \underline{\xi}\right) \\
& +\underline{A}^{d} \underline{\bar{\xi}}^{d} \underline{\Omega}^{\tilde{\alpha}-1} C\left(\underline{Y}, \underline{q}^{u}, \underline{\xi}\right)^{\frac{\tilde{\alpha}}{1-\tilde{\alpha}} \tilde{\sigma}^{-1}}\left(\frac{1}{\underline{q}^{u}} \frac{\underline{\Omega}}{1-\widetilde{\alpha}}\right) \\
& =\bar{C}^{\tilde{\sigma}^{-1}} C\left(\underline{Y}, \underline{q}^{u}, \underline{\xi}\right)^{-\tilde{\sigma}^{-1}} C_{q^{u}}\left(\underline{Y}, \underline{q^{u}}, \underline{\xi}\right)\left(1+\frac{\tilde{\sigma}^{-1}}{1-\tilde{\alpha}} \underline{\Omega} C\left(\underline{Y}, \underline{q}^{u}, \underline{\xi}\right)^{\frac{\tilde{\sigma}^{-1}}{1-\tilde{\alpha}}-1} \frac{1}{1+s^{d}}\right) \\
& +\frac{\underline{A}^{d}}{1-\widetilde{\widetilde{\alpha}}} \underline{\Omega}^{\tilde{\alpha}} C\left(\underline{Y}, \underline{q}^{u}, \underline{\xi}\right)^{\frac{\tilde{\alpha}}{1-\tilde{\alpha}}} \tilde{\tilde{\sigma}}^{-1}
\end{aligned}
$$

Implicitly differentiating (32) we obtain

$$
C_{q^{u}}\left(\underline{Y}, \underline{q}^{u}, \underline{\xi}\right)=\frac{-\frac{\partial \Omega\left(\underline{q}^{u}, \underline{\xi}\right)}{\partial q} C\left(\underline{Y}, \underline{q}^{u}, \underline{\xi}\right)^{\frac{\tilde{\sigma}^{-1}}{1-\tilde{\alpha}}}}{1+\chi^{*}}
$$

where $\chi^{*}=\frac{\widetilde{\sigma}^{-1}}{1-\widetilde{\alpha}} \underline{\Omega} C\left(\underline{Y}, \underline{q}^{u}, \underline{\xi}\right)^{\frac{\widetilde{\sigma}^{-1}}{1-\widetilde{\alpha}}-1}>0$ and $\frac{\partial \Omega\left(\underline{q}^{u}, \underline{\xi}\right)}{\partial q^{u}}=\frac{1}{\underline{q}^{u}} \frac{1}{1-\widetilde{\alpha}} \underline{\Omega}$. Using this we can write

$$
\begin{aligned}
U_{q^{u}}\left(\underline{Y}, \underline{q}^{u}, \underline{\xi}\right) & =-\bar{C}^{\tilde{\sigma}^{-1}} C\left(\underline{Y}, \underline{q}^{u}, \underline{\xi}\right)^{\frac{\alpha}{1-\tilde{\alpha}} \tilde{\sigma}^{-1}} \frac{1+\frac{\chi^{*}}{1+s^{d}}}{1+\chi^{*}} \frac{1}{\underline{q}^{u}} \frac{1}{1-\widetilde{\alpha}} \underline{\Omega} \\
& +\frac{\underline{A}^{d}}{1-\widetilde{\alpha}} \underline{\Omega}^{\tilde{\alpha}} C\left(\underline{Y}, \underline{q}^{u}, \underline{\xi}\right)^{\frac{\tilde{\alpha}}{1-\tilde{\alpha}} \tilde{\sigma}^{-1}} \\
& =-\bar{C}^{\tilde{\sigma}^{-1}} C\left(\underline{Y}, \underline{q}^{u}, \underline{\xi}\right)^{\frac{\alpha}{1-\tilde{\alpha}} \tilde{\sigma}^{-1}} \frac{1}{\underline{q}^{u}} \frac{1}{1-\widetilde{\alpha}} \underline{\Omega}\left(\frac{1+\frac{\chi^{*}}{1+s^{d}}}{1+\chi^{*}}-\frac{\underline{1}}{1+s^{d}}\right) \underline{\Omega} \\
& =-\bar{C}^{\tilde{\sigma}^{-1}} C\left(\underline{Y}, \underline{q}^{u}, \underline{\xi}\right)^{\frac{\alpha}{1-\tilde{\alpha}} \tilde{\sigma}^{-1}} \frac{1}{\underline{q}^{u}} \frac{1}{1-\widetilde{\alpha}} \underline{\Omega}\left(\frac{s^{d}}{\left(1+\chi^{*}\right)\left(1+s^{d}\right)}\right) \underline{\Omega}
\end{aligned}
$$

proving that $U_{q}<0$ for $s^{d}>0, U_{q}>0$ for $s^{d}<0$, and $U_{q}=0$ for $s^{d}=0$. For $\underline{\tau}$ sufficiently close to $\underline{\tau}^{e f f}\left(s^{d}\right)$ we furthermore have from lemma 1 that $U_{Y}$ is sufficiently close to zero, so that (66) implies that $\underline{\Gamma}_{1}$ is also sufficiently to zero, so that from (70) it follows that $\underline{\Psi}$ has the same sign as $U_{q}$, whenever $\underline{\tau} \neq \underline{\tau}^{\text {eff }}$. Furthermore, for $\underline{\tau}=\underline{\tau}^{\text {eff }}$ we have $U_{Y}=\underline{\Gamma}_{1}=0$ and also $U_{q}=$ 0 for $s^{d}=0$, so that from (70) we obtain $\underline{\Psi}=0$.

\section{A.4 Second Order Conditions for Optimal Allocation}

This appendix establishes that $\left(Y_{t}^{*}, q_{t}^{u *}\right)$, defined in equation (71), satisfy the second-order conditions of optimality. Specifically, we establish that $U_{Y Y}<0, U_{q^{u} q^{u}}<0$ and $U_{Y q^{u}}=0$ holds at the efficient steady state where $s^{d}=0$ and $1-\underline{\tau}=(1-g) \eta /(\eta-1)$. By continuity, second order conditions then also hold for nearby allocations that involving sufficiently small steady state distortions and sufficiently small disturbances. From equation (49) we get

$$
\begin{aligned}
& U_{q^{u}}\left(Y_{t}, \Delta_{t}, q_{t}^{u} ; \xi_{t}\right) \\
& =C_{q^{u}}\left(Y_{t}, q_{t}^{u}, \xi_{t}\right)\left(\begin{array}{l}
\bar{C}_{t}^{\tilde{\sigma}^{-1}} C\left(Y_{t}, q_{t}^{u}, \xi_{t}\right)^{-\tilde{\sigma}^{-1}} \\
+\frac{\tilde{\sigma}^{-1}}{1-\tilde{\alpha}} A_{t}^{d} \bar{\xi}_{t}^{d} \Omega\left(q_{t}^{u}, \xi_{t}\right)^{\tilde{\alpha}} C\left(Y_{t}, q_{t}^{u}, \xi_{t}\right)^{\frac{\tilde{\alpha}}{1-\tilde{\alpha}} \tilde{\sigma}^{-1}-1}
\end{array}\right) \\
& +A_{t}^{d} \bar{\xi}_{t}^{d} \Omega\left(q_{t}^{u}, \xi_{t}\right)^{\tilde{\alpha}-1} \frac{1}{\tilde{\alpha}-1} \frac{\Omega\left(q_{t}^{u}, \xi_{t}\right)}{q_{t}^{u}} C\left(Y_{t}, q_{t}^{u}, \xi_{t}\right)^{\frac{\tilde{\alpha}}{1-\tilde{\alpha}} \tilde{\sigma}^{-1}}
\end{aligned}
$$


Using

$$
C_{q^{u}}\left(Y_{t}, q_{t}^{u} ; \xi_{t}\right)=\frac{-\frac{1}{q_{t}^{u}} \frac{1}{1-\widetilde{\alpha}} \Omega\left(q_{t}^{u}, \xi_{t}\right) C\left(Y_{t}, q_{t}^{u}, \xi_{t}\right)^{\chi+1}}{1+(1+\chi) \Omega\left(q_{t}^{u}, \xi_{t}\right) C\left(Y_{t}, q_{t}^{u}, \xi_{t}\right)^{\chi}}
$$

where

$$
\chi \equiv \frac{\tilde{\sigma}^{-1}}{1-\tilde{\alpha}}-1
$$

we get

$$
U_{q^{u}}\left(Y_{t}, \Delta_{t}, q_{t}^{u} ; \xi_{t}\right)=\frac{\frac{1}{q_{t}^{u}} \frac{1}{1-\widetilde{\alpha}} \Omega\left(q_{t}^{u}, \xi_{t}\right)}{1+(1+\chi) \Omega\left(q_{t}^{u}, \xi_{t}\right) C\left(Y_{t}, q_{t}^{u}, \xi_{t}\right) \chi} \bar{C}_{t}^{\tilde{\sigma}^{-1}}\left(\frac{\bar{\xi}_{t}^{d}}{\left(1+s^{d}\right) q_{t}^{u}}-1\right) .
$$

We have $U_{q^{u}}=0$ if and only if

$$
q_{t}^{u *}=\frac{\bar{\xi}_{t}^{d}}{\left(1+s^{d}\right)}
$$

At the efficient steady state, we have $s^{d}=0$ and thus $\underline{q}^{u}=\underline{\xi}^{d}=q_{t}^{u *}$, so that the term in the last parenthesis in (132) is zero. Evaluating $U_{q^{u} q^{u}}$ at such a steady state delivers

$$
U_{q^{u} q^{u}}=\frac{\frac{1}{\underline{q}^{u}} \frac{1}{1-\widetilde{\alpha}} \Omega\left(\underline{q}^{u}, \underline{\xi}\right)}{1+(1+\chi) \Omega\left(\underline{q}^{u}, \underline{\xi}\right) C\left(\underline{Y}, \underline{q}^{u}, \underline{\xi}\right) \chi} \underline{C}^{\tilde{\sigma}^{-1}}\left(-\frac{\underline{\bar{\xi}}^{d}}{\left(1+s^{d}\right)} \frac{1}{\left(\underline{q}^{u}\right)^{2}}\right)<0 .
$$

From equation (49) we also get

$$
\begin{aligned}
U_{Y}\left(Y_{t}, \Delta_{t}, q_{t}^{u} ; \xi_{t}\right) & =\bar{C}_{t}^{\tilde{\sigma}^{-1}} C\left(Y_{t}, q_{t}^{u}, \xi_{t}\right)^{-\tilde{\sigma}^{-1}} C_{Y}\left(Y_{t}, q_{t}^{u}, \xi_{t}\right) \\
& -\frac{\lambda}{1+\nu}(1+\omega) \frac{\bar{H}_{t}^{-\nu}}{A_{t}^{1+\omega}} Y_{t}^{\omega} \Delta_{t} \\
& +\frac{A_{t}^{d} \bar{\xi}_{t}^{d}}{\tilde{\alpha}} \Omega\left(q_{t}^{u}, \xi_{t}\right)^{\tilde{\alpha}}\left(\frac{\tilde{\alpha}}{1-\tilde{\alpha}} \tilde{\sigma}^{-1}\right) C\left(Y_{t}, q_{t}^{u}, \xi_{t}\right)^{\frac{\tilde{\alpha}}{1-\tilde{\alpha}} \tilde{\sigma}^{-1}-1} C_{Y}\left(Y_{t}, q_{t}^{u}, \xi_{t}\right)
\end{aligned}
$$

Using

$$
C_{Y}\left(Y_{t}, q_{t}^{u}, \xi_{t}\right)=\frac{1-g_{t}}{1+\Omega\left(q_{t}^{u}, \xi_{t}\right)(1+\chi) C\left(Y_{t}, q_{t}^{u}, \xi_{t}\right)^{\chi}}>0
$$

together with

$$
A_{t}^{d} \bar{\xi}_{t}^{d} \Omega\left(q_{t}^{u}, \xi_{t}\right)^{\tilde{\alpha}}=\frac{\Omega\left(q_{t}^{u}, \xi_{t}\right)}{1+s^{d}} \bar{C}_{t}^{\tilde{\sigma}^{-1}}
$$

and (133), we obtain

$$
\begin{aligned}
U_{Y}\left(Y_{t}, \Delta_{t}, q_{t}^{u} ; \xi_{t}\right) \\
=\bar{C}_{t}^{\tilde{\sigma}^{-1}} C\left(Y_{t}, q_{t}^{u}, \xi_{t}\right)^{-\tilde{\sigma}^{-1}}\left(1-g_{t}\right) \frac{1+\Omega\left(q_{t}^{u}, \xi_{t}\right)(1+\chi) C\left(Y_{t}, q_{t}^{u}, \xi_{t}\right)^{\chi}\left(\frac{q_{t}^{u *}}{q_{t}^{u}}\right)}{1+\Omega\left(q_{t}^{u}, \xi_{t}\right)(1+\chi) C\left(Y_{t}, q_{t}^{u}, \xi_{t}\right)^{\chi}} \\
\\
\quad-\frac{\lambda}{1+\nu}(1+\omega) \frac{\bar{H}_{t}^{-\nu}}{A_{t}^{1+\omega}} Y_{t}^{\omega} \Delta_{t} .
\end{aligned}
$$

Differentiating once more w.r.t. $Y_{t}$ and evaluating at the steady state where $q^{u}=q^{u *}$, one obtains

$$
U_{Y Y}=-\tilde{\sigma}^{-1}(1-\underline{g}) \underline{\bar{C}}^{\tilde{\sigma}^{-1}} C\left(\underline{Y}, \underline{q}^{u}, \underline{\xi}\right)^{-\tilde{\sigma}^{-1}-1} C_{Y}-\frac{\lambda}{1+\nu}(1+\omega) \omega \frac{\underline{\bar{H}}^{-\nu}}{\underline{A}^{1+\omega}} \underline{Y}^{\omega-1}<0 .
$$


Differentiating (136) w.r.t. $q_{t}^{u}$ and one obtains

$$
\begin{aligned}
U_{Y q^{u}}\left(Y_{t}, \Delta_{t}, q_{t}^{u} ; \xi_{t}\right) \\
\quad=-\tilde{\sigma}^{-1} \bar{C}_{t}^{\tilde{\sigma}^{-1}} C\left(Y_{t}, q_{t}^{u}, \xi_{t}\right)^{-\tilde{\sigma}^{-1}-1} C_{q^{u}}\left(Y_{t}, q_{t}^{u}, \xi_{t}\right)\left(1-g_{t}\right) \\
\quad-\bar{C}_{t}^{\tilde{\sigma}^{-1}} C\left(Y_{t}, q_{t}^{u}, \xi_{t}\right)^{-\tilde{\sigma}^{-1}}\left(1-g_{t}\right) \frac{\Omega\left(q_{t}^{u}, \xi_{t}\right)(1+\chi) C\left(Y_{t}, q_{t}^{u}, \xi_{t}\right)^{\chi} \frac{q_{t}^{u *}}{\left(q_{t}^{u}\right)^{2}}}{1+\Omega\left(q_{t}^{u}, \xi_{t}\right)(1+\chi) C\left(Y_{t}, q_{t}^{u}, \xi_{t}\right)^{\chi}}
\end{aligned}
$$

Using (131) and evaluating at a steady state with $\underline{q}^{u}=\underline{q}^{u *}$ one obtains

$$
U_{Y q^{u}}=0 .
$$

\section{A.5 Linearized Phillips Curve}

This appendix shows that the linearized structural equations (87) imply the generalized Phillips curve (90) and derives the values of the Phillips curve coefficients. Premultiplying equations (87) by $(1,-1)$ and using the fact that this is a left eigenvector of $D(1)$, we obtain

$$
\begin{aligned}
\widehat{F}_{t}-\widehat{K}_{t} & =(1-\alpha \beta)\left[-\Theta+\left(f_{y}-k_{y}\right) y_{t}^{g a p}+f_{q}\left(\widehat{q}_{t}^{u}-\widehat{q}_{t}^{u *}\right)+\left(f_{\xi}^{\prime}-k_{\xi}^{\prime}\right)\left(\xi_{t}-\underline{\xi}\right)\right] \\
& +\beta E_{t}\left[\widehat{F}_{t+1}-\widehat{K}_{t+1}\right]+\alpha \beta\left[M_{1}-M_{2}\right] \zeta_{t}
\end{aligned}
$$

To a first-order approximation, equation (45) implies that

$$
\pi_{t}=\frac{1-\alpha}{\alpha} \frac{1}{1+\omega \eta}\left(\widehat{K}_{t}-\widehat{F}_{t}\right) .
$$

Substituting the preceding equation into (138), one obtains (90) where the coefficients $\kappa$ are given by

$$
\begin{aligned}
\kappa_{y} & =\frac{1-\alpha}{\alpha} \frac{1-\alpha \beta}{1+\omega \eta}\left(k_{y}-f_{y}\right) \\
\kappa_{q} & =-\frac{1-\alpha}{\alpha} \frac{1-\alpha \beta}{1+\omega \eta} f_{q} \\
\kappa_{\zeta}^{\prime} & =\frac{(1-\alpha) \beta}{1+\omega \eta}\left[M_{2}-M_{1}\right] .
\end{aligned}
$$

From $f_{q}<0$ we obtain $\kappa_{q}>0$. From (125) we obtain $\kappa_{y}>0$.

\section{A.6 Quadratically Approximated Welfare Objective}

This appendix derives the result stated in equation (94). We can write

$$
\begin{aligned}
& \sum_{t=0}^{\infty} \beta^{t}\left[U\left(Y_{t}, \Delta_{t}, q_{t}^{u} ; \xi_{t}\right)+\gamma_{t}\left(\tilde{h}\left(\Delta_{t-1}, K_{t} / F_{t}\right)-\Delta_{t}\right)\right] \\
& =\sum_{t=0}^{\infty} \beta^{t}\left[U\left(Y_{t}, \Delta_{t}, q_{t}^{u} ; \xi_{t}\right)+\gamma_{t}\left(h\left(\Delta_{t-1}, \Pi_{t}\right)-\Delta\right] .\right.
\end{aligned}
$$

Since $U_{Y}=U_{q^{u}}=U_{Y q^{u}}=0$ and since $U_{\Delta}+\gamma\left(\beta h_{1}-1\right)=0$ at the optimal steady state and given our assumption that $\Delta_{-1} \sim O\left(\sigma^{2}\right)$, which implies $\Delta_{t} \sim O\left(\sigma^{2}\right)$ for all $t \geq 0$, a second order approximation of the contribution of the variables $\left(Y_{t}, \Delta_{t}, q_{t}^{u}, \Delta_{t}, \Pi_{t}, \xi_{t}\right)$ to household utility is given by

$$
\frac{1}{2} U_{\widehat{Y} \widehat{Y}}\left(\widehat{Y}_{t}-\widehat{Y}_{t}^{*}\right)+\frac{1}{2} U_{\widehat{q}^{u}} \widehat{q}^{u}\left(\widehat{q}_{t}^{u}-\widehat{q}_{t}^{u *}\right)+\frac{1}{2} \gamma^{*} h_{22} \pi_{t}^{2}+\text { t.i.p. }
$$


where t.i.p. denotes terms independent of policy and where we used the fact that $h_{2} \equiv \frac{\partial h(\Delta, \Pi)}{\partial \Pi}=$ 0 at the optimal steady state. The approximation coefficients are given by $U_{\widehat{Y} \widehat{Y}} \equiv Y \frac{\partial}{\partial Y}\left(U_{\widehat{Y}}\right) \equiv$ $Y \frac{\partial}{\partial Y}\left(Y U_{Y}\right)=\underline{Y}^{*} U_{Y}+\left(\underline{Y}^{*}\right)^{2} U_{Y Y}$ and correspondingly for $U_{\widehat{q}^{u} \widehat{q}^{u}}$. Evaluated at the optimal steady state where $U_{Y}=U_{q^{u}}=0$, we get

$$
\begin{aligned}
& \Lambda_{\pi}=-\frac{1}{2} \underline{\gamma}^{*} h_{22}>0 \\
& \Lambda_{y}=-\frac{1}{2}\left(\underline{Y}^{*}\right)^{2} U_{Y Y}>0 \\
& \Lambda_{q}=-\frac{1}{2}\left(\underline{q}^{u *}\right)^{2} U_{q^{u} q^{u}}>0,
\end{aligned}
$$

where explicit expressions for $U_{q^{u} q^{u}}$ and $U_{Y Y}$ are given in (134) and (137), respectively, and $h_{2} \equiv \frac{\partial^{2} h(\Delta, \Pi)}{(\partial \Pi)^{2}}$ and $\underline{\gamma}^{*}$ are given by

$$
\begin{aligned}
h_{22} & =\frac{\alpha \eta(1+\omega)(1+\omega \eta)}{1-\alpha}>0 \\
\underline{\gamma}^{*} & =\frac{U_{\Delta}}{1-a \beta}<0,
\end{aligned}
$$

with

$$
\begin{aligned}
U_{\Delta} & =-\frac{\lambda}{1+\nu} \underline{\bar{H}}^{-\nu}\left(\frac{\underline{Y}^{*}}{\underline{A}}\right)^{1+\omega} \\
& =-\frac{\underline{Y}^{*}(1-\underline{g})}{1+\omega}\left(\frac{\bar{C}^{\tilde{\sigma}^{-1}}}{C\left(\underline{Y}^{*}, \underline{q}^{u *}, \underline{\xi}\right)}\right)^{\tilde{\sigma}^{-1}}<0,
\end{aligned}
$$

where the last line follows from (124) and the property that the wedge in (68) satisfies $\Theta=0$ at the efficient steady state.

\section{A.7 Details of the LQ Approximate Problem}

This appendix derives the coefficients $(a, b, H)$ of the Lagrangian $L^{u}$ in equation (105) and shows that $H$ is positive definite for sufficiently large values of $\theta / \sigma^{2}$.

The Lagrangian $L^{u}$ captures the period $t$ terms that are unpredictable as of period $t-1$ in the Lagrangian associated with minimizing the loss function (100) subject to the constraints (101) and (102). These terms are given by

$$
\begin{aligned}
L^{u} & =E_{0} \sum_{t=1}^{\infty} \beta^{t}\left\{\begin{array}{c}
\frac{1}{2} \sum_{k}\left(\Lambda_{\pi}\left(\pi_{0, k}\right)^{2}+\Lambda_{y}\left(y_{0, k}\right)^{2}+\Lambda_{q}\left(q_{0, k}-q_{0, k}^{*}\right)^{2}\right. \\
-\varphi_{t} \sum_{k}\left(\pi_{0, k}-\kappa_{y} y_{0, k}-\kappa_{q}\left(q_{0, k}-q_{0, k}^{*}\right)\right) e_{k, t} \\
-\psi_{t} \sum_{k}\left(q_{0, k}-q_{0, k}^{*}\right) e_{k, t}
\end{array}\right\} \\
& +E_{0} \sum_{t=0}^{\infty} \beta^{t}\left\{\frac{\beta}{\theta}\left(\varphi_{t},(1-\delta) \psi_{t}\right) S \widehat{\zeta}_{t}-\frac{\beta}{2 \theta} \widehat{\zeta}_{t}^{\prime} S \widehat{\zeta}_{t}\right\} \\
& + \text { t.i.p. }
\end{aligned}
$$

where t.i.p. summarizes additional terms that are independent of policy, $q_{0, k}^{*}$ denotes the reaction coefficient of $\widehat{q}_{t}^{u *}$ to the $k$-th disturbance $\left(e_{k t}\right)$ and

$$
S=\left(\begin{array}{cc}
\sum_{k}\left(\pi_{0, k}\right)^{2} & \sum_{k} \pi_{k} q_{0, k} \\
\sum_{k} \pi_{0, k} q_{0, k} & \sum_{k}\left(q_{0, k}\right)^{2}
\end{array}\right) .
$$


The coefficients $a$ and $b$ are thus given by

$$
\begin{aligned}
a_{t, k} & \equiv\left(\begin{array}{c}
-\varphi_{t} \\
-\Lambda_{q} q_{0, k}^{*}+\varphi_{t} \kappa_{q}-\psi_{t}
\end{array}\right) \\
b_{t} & \equiv \varphi_{t} \kappa_{y} .
\end{aligned}
$$

One can furthermore write

$$
\begin{aligned}
& E_{0} \sum_{t=0}^{\infty} \beta^{t}\left\{\frac{\beta}{\theta}\left(\varphi_{t},(1-\delta) \psi_{t}\right) S \widehat{\zeta}_{t}-\frac{\beta}{2 \theta} \widehat{\zeta}_{t}^{\prime} S \widehat{\zeta}_{t}\right\} \\
& =\frac{\beta}{1-\beta}\left\{\begin{array}{l}
\frac{1}{\theta} \operatorname{tr}\left(S(1-\beta) E_{0} \sum_{t=0}^{\infty} \beta^{t} \widehat{\zeta}_{t}\left(\varphi_{t},(1-\delta) \psi_{t}\right)\right) \\
-\frac{1}{2 \theta} \operatorname{tr}\left(S(1-\beta) E_{0} \sum_{t=0}^{\infty} \beta^{t} \widehat{\zeta}_{t} \widehat{\zeta}_{t}^{\prime}\right.
\end{array}\right\} .
\end{aligned}
$$

Defining

$$
\begin{aligned}
E_{\zeta^{\pi} \varphi} & \equiv(1-\beta) E_{0} \sum_{t=0}^{\infty} \zeta_{t}^{\pi} \varphi_{t} \\
E_{\zeta^{\pi} \psi} & \equiv(1-\beta) E_{0}^{t} \sum_{t=0}^{\infty} \zeta_{t}^{\pi} \psi_{t} \\
E_{\zeta^{q} \psi} & \equiv(1-\beta) E_{0} \sum_{t=0}^{\infty} \beta_{t}^{t} \zeta_{t}^{q} \psi_{t} \\
E_{\zeta^{q} \varphi} & \equiv(1-\beta) E_{0} \sum_{t=0}^{\infty} \beta^{t} \zeta_{t}^{q} \varphi_{t} \\
E_{\zeta^{\pi} \zeta^{\pi}} & \equiv(1-\beta) E_{0}^{t} \sum_{t=0}^{\infty} \zeta_{t}^{\pi} \zeta_{t}^{\pi} \\
E_{\zeta^{\pi} \zeta^{q}} & \equiv(1-\beta) E_{0}^{t} \sum_{t=0}^{\infty} \zeta_{t}^{\pi} \zeta_{t}^{q} \\
E_{\zeta^{q} \zeta^{q}} & \equiv(1-\beta) E_{0}^{t} \sum_{t=0}^{\infty} \zeta_{t}^{q} \zeta_{t}^{q}
\end{aligned}
$$

and using (140), the quadratic terms in (139) can be written as

$$
\frac{\beta}{1-\beta}\left\{\begin{array}{l}
\frac{1}{2} \sum_{k}\left(\Lambda_{\pi}\left(\pi_{0, k}\right)^{2}+\Lambda_{y}\left(y_{0, k}^{g a p}\right)^{2}+\Lambda_{q}\left(q_{0, k}\right)^{2}\right) \\
+\frac{1}{\theta} \sum_{k}\left(\pi_{0, k}, q_{0, k}\right)\left(\left(\begin{array}{cc}
E_{\zeta^{\pi} \varphi} & (1-\delta) E_{\zeta^{\pi} \psi} \\
E_{\zeta^{q} \varphi} & (1-\delta) E_{\zeta^{q} \psi}
\end{array}\right)-\frac{1}{2}\left(\begin{array}{cc}
E_{\zeta^{\pi} \zeta^{\pi}} & E_{\zeta^{\pi} \zeta^{q}} \\
E_{\zeta^{\pi} \zeta^{q}} & E_{\zeta^{q} \zeta^{q}}
\end{array}\right)\right)\left(\pi_{0, k}, q_{0, k}\right)^{\prime}
\end{array}\right\},
$$

which implies

$$
H \equiv\left(\begin{array}{ll}
\frac{\Lambda_{\pi}}{2}+\frac{1}{\theta}\left(E_{\zeta^{\pi} \varphi}-\frac{1}{2} E_{\zeta^{\pi} \zeta^{\pi}}\right) & \frac{1}{\theta}\left((1-\delta) E_{\zeta^{\pi} \psi}-\frac{1}{2} E_{\zeta^{\pi} \zeta^{q}}\right) \\
\frac{1}{\theta}\left(E_{\zeta^{q} \varphi}-\frac{1}{2} E_{\zeta^{\pi} \zeta^{q}}\right) & \left.\frac{\Lambda_{q}}{2}+\frac{1}{\theta}(1-\delta) E_{\zeta^{q} \psi}-\frac{1}{2} E_{\zeta^{q} \zeta^{q}}\right)
\end{array}\right) .
$$

For the limit $\sigma^{2} / \theta \rightarrow 0$, the $H$ matrix converges to

$$
\left(\begin{array}{ll}
\frac{\Lambda_{\pi}}{2} & 0 \\
0 & \frac{\Lambda_{q}}{2}
\end{array}\right)
$$

which is positive definite because $\Lambda_{\pi}>0$ and $\Lambda_{q}>0$.

\section{A.8 Multipliers in the Nonlinear Best-Response Problem}

The FOCs of the nonlinear best-response problem (91) are given by

$$
\begin{aligned}
& U_{Y}\left(Y_{t}, \Delta_{t}, q_{t}^{u} ; \xi_{t}\right)+\Gamma_{t}^{\prime} z_{Y}\left(Y_{t}, q_{t}^{u}, \xi_{t}\right)=0 \\
&-\gamma_{t} \tilde{h}_{2}\left(\Delta_{t-1}, K_{t} / F_{t}\right) \frac{K_{t}}{F_{t}^{2}}-\Gamma_{1 t}+\alpha m_{t} \Gamma_{t-1}^{\prime} D_{1}\left(K_{t} / F_{t}\right)=0 \\
& \gamma_{t} \tilde{h}_{2}\left(\Delta_{t-1}, K_{t} / F_{t}\right) \frac{1}{F_{t}}-\Gamma_{2 t}+\alpha m_{t} \Gamma_{t-1}^{\prime} D_{2}\left(K_{t} / F_{t}\right)=0 \\
& U_{\Delta}\left(Y_{t}, \Delta_{t}, q_{t}^{u} ; \xi_{t}\right)-\gamma_{t}+\beta E_{t}\left[\gamma_{t+1} \tilde{h}_{1}\left(\Delta_{t}, K_{t+1} / F_{t+1}\right)\right]=0 \\
& U_{q}\left(Y_{t}, \Delta_{t}, q_{t}^{u}, \xi_{t}\right)+\Gamma_{t}^{\prime} z_{q}\left(Y_{t}, q_{t}^{u}, \xi_{t}\right)+\Psi_{t-1}(1-\delta) m_{t}-\Psi_{t}=0
\end{aligned}
$$


for all $t \geq 0$. At the optimal steady state, we have $U_{Y}=\underline{\Gamma}=0$, see section 4.3 , and $\underline{\Psi}=0$, see lemma 2. Given the assumptions in (74) and the assumed disturbance process (73), the endogenous variables $\left(Y_{t}, \Delta_{t}, q_{t}^{u}, K_{t} / F\right)$ will deviate from their optimal steady sate value only by a gap of order $O(\sigma)$. Equation (141) then implies that $\Gamma_{t}=\left(\Gamma_{1 t},-\Gamma_{1 t}\right)$ is of order $O(\sigma)$, as claimed. Likewise, equation (144) implies that $\gamma_{t}$ will deviate from its optimal steady state value $\gamma^{*}<0$ only by a gap of order $O(\sigma)$. Equation (145) then implies that $\Psi_{t}$ is of order $O(\sigma)$, provided the initial precommitment satisfies $\Psi_{-1} \sim O(\sigma)$, as assumed, and the belief distortions satisfy $m_{t} \sim O(1)$. The latter follows from equation (15) and the facts $\left(\zeta_{t}^{\pi}, \zeta_{t}^{q}\right) \sim O(\sigma)$, $x_{t+1}=\left(\pi_{t+1}, \widehat{q}_{t+1}^{u}\right) \sim O(\sigma)$ and $\theta \sim O\left(\sigma^{2}\right)$.

\section{A.9 Worst-Case Distortions for the Targeting Rule (123)}

The worst-case belief distortion problem is given by

$$
\max _{\left\{\widehat{\zeta}_{t}, \pi_{t}, y_{t}^{g a p}, \widehat{q}_{t}^{u}\right\}_{t=0}^{\infty}} E_{0} \sum_{t=0}^{\infty} \frac{\beta^{t}}{2}\left[\Lambda_{\pi} \pi_{t}^{2}+\Lambda_{y}\left(y_{t}^{g a p}\right)^{2}+\Lambda_{q}\left(\widehat{q}_{t}^{u}-\widehat{q}_{t}^{u *}\right)^{2}-\frac{\beta}{\theta} \widehat{\zeta}_{t}^{\prime} V \widehat{\zeta}_{t}\right]
$$

subject to the constraints

$$
\begin{gathered}
\pi_{t}=\beta E_{t} \pi_{t+1}+\kappa_{y} y_{t}^{g a p}+\kappa_{q}\left(\widehat{q}_{t}^{u}-\widehat{q}_{t}^{u *}\right)+u_{t} \\
+\beta \theta^{-1} V_{1} \widehat{\zeta}_{t} \\
\left(\widehat{q}_{t}^{u}-\widehat{q}_{t}^{u *}\right)=\beta(1-\delta) E_{t}\left[\widehat{q}_{t+1}^{u}-\widehat{q}_{t+1}^{u *}\right]+(1-\beta(1-\delta)) s^{d} \\
+\beta(1-\delta) \theta^{-1} V_{2} \widehat{\zeta}_{t}
\end{gathered}
$$

for all $t \geq 0$, subject to the proposed target criterion

$$
\begin{aligned}
0 & =\pi_{t}+\frac{\Lambda_{y}}{\Lambda_{\pi} \kappa_{y}}\left(y_{t}^{g a p}-y_{t-1}^{g a p}\right) \\
& +\frac{\theta^{-1}}{\Lambda_{\pi}} E_{\varphi \varphi}\left(\pi_{t}-E_{t-1} \pi_{t}\right)+\frac{\theta^{-1}}{\Lambda_{\pi}}(1-\delta) E_{\varphi \psi}\left(\widehat{q}_{t}^{u}-E_{t-1} \widehat{q}_{t}^{u}\right)
\end{aligned}
$$

for all $t \geq 1$,and subject to an initial precommitment for $t=0$ of the form

$$
\frac{\varphi_{-1}}{\psi_{-1}} \pi_{0}+(1-\delta)\left(\widehat{q}_{0}^{u}-\widehat{q}_{0}^{u *}\right)=G_{0}
$$

where the coefficients $\varphi_{-1} / \psi_{-1}$ and $G_{0}$ are taken as given. The matrix $V$ satisfies

$$
V=\Phi(V)
$$

where the function $\Phi(\cdot)$ is defined in Appendix A.10. Appendix A.10 furthermore provides a sufficient condition, see equation (157), which insures that there is a unique $V$ solving (151) for belief distortions $\left\{\zeta_{t}^{\pi}, \zeta_{t}^{q}\right\}_{t=0}^{\infty}$ in the neighborhood of the upper bound distortions. The same condition also insures that that equations (147)-(150) imply a locally unique equilibrium outcome for all belief distortions $\left\{\zeta_{t}^{\pi}, \zeta_{t}^{q}\right\}_{t=0}^{\infty}$ in the neighborhood of the upper bound distortions. We proceed under the assumption that the sufficient condition (157) is satisfied.

The Lagrangian of the worst-case problem is 


$$
\begin{aligned}
& \max _{\left\{\widehat{\zeta}_{t}, \pi_{t}, y_{t}^{g a p}, \widehat{q}_{t}^{u}\right\}_{t=0}^{\infty}\left\{\psi_{t-1}, \varphi_{t}, \eta_{t+1}\right\}_{t=0}^{\infty}} E_{0} \sum_{t=0}^{\infty} \frac{\beta^{t}}{2}\left[\Lambda_{\pi} \pi_{t}^{2}+\Lambda_{y}\left(y_{t}^{g a p}\right)^{2}+\Lambda_{q}\left(\widehat{q}_{t}^{u}-\widehat{q}_{t}^{u *}\right)^{2}-\frac{\beta}{\theta} \widehat{\zeta}_{t}^{\prime} V \widehat{\zeta}_{t}\right] \\
& +\sum_{t=0}^{\infty} \beta^{t}\left[\begin{array}{l}
\varphi_{t}\left(-\pi_{t}+\beta E_{t} \pi_{t+1}+\kappa_{y} y_{t}^{g a p}+\kappa_{q}\left(\widehat{q}_{t}^{u}-\widehat{q}_{t}^{u *}\right)+u_{t}+\beta \theta^{-1} V_{1} \widehat{\zeta}_{t}\right) \\
+\psi_{t}\left(-\left(\widehat{q}_{t}^{u}-\widehat{q}_{t}^{u *}\right)+\beta(1-\delta) E_{t}\left[\widehat{q}_{t+1}^{u}-\widehat{q}_{t+1}^{u *}\right]+(1-\beta(1-\delta)) s^{d}+\beta(1-\delta) \theta^{-1} V_{2} \widehat{\zeta}_{t}\right) \\
+\beta \eta_{t+1}\left(\pi_{t+1}+\frac{\Lambda_{y}}{\Lambda_{\pi} \kappa_{y}}\left(y_{t+1}^{g a p}-y_{t}^{g a p}\right)+\frac{\theta^{-1}}{\Lambda_{\pi}} E_{\varphi \varphi}\left(\pi_{t+1}-E_{t} \pi_{t+1}\right)+\frac{\theta^{-1}}{\Lambda_{\pi}}(1-\delta) E_{\varphi \psi}\left(\widehat{q}_{t+1}^{u}-E_{t} \widehat{q}_{t+1}^{u}\right)\right)
\end{array}\right. \\
& +\psi_{-1}\left(\begin{array}{l}
\left.\frac{\varphi_{-1}}{\psi_{-1}} \pi_{0}+(1-\delta)\left(\widehat{q}_{0}^{u}-\widehat{q}_{0}^{u *}\right)-G_{0}\right)
\end{array}\right.
\end{aligned}
$$

where $\left(\varphi_{-1} / \psi_{-1}, G_{0}\right)$ are given by the initial precommitment, $\eta_{t+1}$ is the Lagrange multiplier on the policy commitment in period $t+1$, and $\psi_{-1}$ the multiplier on the initial precommitment. All choice variables $X$ take the form $X_{t+1}=E_{t} X_{t+1}+\sum_{k} X_{0, k} e_{k, t+1}$.

The Lagrangian (152) can be written as the sum $L^{u}+L^{e}$, where $L^{e}$ depends on $\left(\widehat{\zeta}_{0}, \pi_{0}, y_{0}^{g a p}, \widehat{q}_{0}^{u}, \psi_{-1}, \psi_{0}, \varphi\right.$ and the predictable components $\left\{E_{t} \widehat{\zeta}_{t+1}, E_{t} \pi_{t+1}, E_{t} y_{t+1}^{g a p}, E_{t} \widehat{q}_{t+1}^{u}, E_{t} \psi_{t+1}, E_{t} \varphi_{t+1}, E_{t} \eta_{t+1}\right\}_{t=0}^{\infty}$ and $L^{u}$ on the surprise components in $t$. The first-order condition with respect to $\widehat{\zeta}_{0}$ and $E_{t} \widehat{\zeta}_{t+1}$ are

$$
\begin{aligned}
V \widehat{\zeta}_{0}+\varphi_{0} V_{1}^{\prime}+\psi_{0}(1-\delta) V_{2}^{\prime} & =0 \\
V E_{t-1} \widehat{\zeta}_{t}+E_{t-1} \varphi_{t} V_{1}^{\prime}+E_{t-1} \psi_{t}(1-\delta) V_{2}^{\prime} & =0 \quad \text { for } t \geq 1,
\end{aligned}
$$

which deliver

$$
E_{t-1} \widehat{\zeta}_{t}=E_{t-1}\left(\begin{array}{c}
\varphi_{t} \\
(1-\delta) \psi_{t}
\end{array}\right) \text { for all } t \geq 0
$$

when defining $E_{-1} \widehat{\zeta}_{0}=\widehat{\zeta}_{0}$. Note that condition (153) is also satisfied in the upper bound solution, see equation (111), which suggests that with regard to choosing the predictable components of the belief distortion, the targeting rule (123) is not binding, i.e., $E_{t}\left[\eta_{t+1}\right] \equiv 0$. To verify this conjecture, we derive the FOCs with respect to the forecastable components of inflation, output gap and housing price gap :

$$
\begin{array}{rlrl}
\Lambda_{\pi} \pi_{0}-\varphi_{0}+\varphi_{-1} & =0 & & \\
\Lambda_{\pi} E_{t-1} \pi_{t}-E_{t-1} \varphi_{t}+\varphi_{t-1}-E_{t-1} \eta_{t} & =0 & & \text { for } t \geq 1 \\
\Lambda_{y} y_{0}+\varphi_{0} \kappa_{y}-\beta \frac{\Lambda_{y}}{\Lambda_{\pi} \kappa_{y}} E_{0} \eta_{1} & =0 & & \\
\Lambda_{y} E_{t-1} y_{t}+E_{t-1} \varphi_{t} \kappa_{y}+\frac{\Lambda_{y}}{\Lambda_{\pi} \kappa_{y}}\left(E_{t-1} \eta_{t}-\beta E_{t-1} \eta_{t+1}\right) & =0 & & \text { for } t \geq 1 \\
\Lambda_{q}\left(\widehat{q}_{0}^{u}-\widehat{q}_{0}^{u *}\right)+\varphi_{0} \kappa_{q}-\psi_{0}+(1-\delta) \psi_{-1} & =0 & \\
\Lambda_{q} E_{t-1}\left(\widehat{q}_{t}^{u}-\widehat{q}_{t}^{u *}\right)+E_{t-1} \varphi_{t} \kappa_{q}-E_{t-1} \psi_{t}+(1-\delta) \psi_{t-1} & =0 & & \text { for } t \geq 1
\end{array}
$$

The previous equations together with the predictable parts of the constraints (147)-(148) for $t \geq 0$

$$
\begin{aligned}
E_{t-1} \pi_{t}=\beta E_{t-1} \pi_{t+1}+\kappa_{y} E_{t-1} y_{t}^{g a p}+\kappa_{q} E_{t-1}\left(\widehat{q}_{t}^{u}-\widehat{q}_{t}^{u *}\right)+E_{t-1} u_{t} & \\
& +\beta \theta^{-1} V_{1} E_{t-1} \widehat{\zeta}_{t} \\
E_{t-1}\left(\widehat{q}_{t}^{u}-\widehat{q}_{t}^{u *}\right)=\beta(1-\delta) E_{t-1}\left[\widehat{q}_{t+1}^{u}-\widehat{q}_{t+1}^{u *}\right]+(1-\beta(1-\delta)) s^{d} & +\beta(1-\delta) \theta^{-1} V_{2} E_{t-1} \widehat{\zeta}_{t}
\end{aligned}
$$


and the predictable parts of the targeting rule and the initial commitment

$$
\begin{array}{rlr}
\Lambda_{\pi} E_{t-1} \pi_{t} & =-\frac{\Lambda_{y}}{\kappa_{y}}\left(E_{t-1} y_{t}^{g a p}-y_{t-1}^{g a p}\right) & \text { for } t \geq 1 \\
\frac{\varphi_{-1}}{\psi_{-1}} \pi_{0}+(1-\delta)\left(\widehat{q}_{0}^{u}-\widehat{q}_{0}^{u *}\right) & =G_{0} . &
\end{array}
$$

determine the predictable dynamics of the worst-case problem. It is easy to note that for $E_{t}\left[\eta_{t+1}\right] \equiv 0$, the predictable components are identical to the predictable components of the first-order conditions (113)-(120) describing the upper-bound solution when using the same $V$ matrix . Since there is locally only one solution, the predictable dynamics of the worst-case belief problem must be identical to the predictable dynamics implied by equations (113)-(120). For given $V$ we can thus easily determine the predictable dynamics of the belief distortions.

\section{A.10 Local Uniqueness Around Upper Bound: Sufficient Conditions}

This appendix provides sufficient conditions for there to be a locally unique matrix $V$ for all belief distortions in the neighborhood of the upper bound distortions. It also shows that the same condition implies that the targeting rule (123) and the initial precommitment then deliver a locally unique equilibrium outcome for all belief distortions close to the upper bound distortions.

We prove below the following auxiliary result:

Lemma 3 Consider given belief distortions $\left\{\zeta_{t}^{\pi}, \zeta_{t}^{q}\right\}_{t=0}^{\infty}$ and a given positive semi-definite symmetric matrix $V$. The structural equations (101) and (102), together with the target criterion (123) for $t \geq 1$ and the initial precommitment in period $t=0$ then determine a locally unique equilibrium outcome for $\left\{\pi_{t}, y_{t}^{g a p}, \widehat{q}_{t}^{u}\right\}_{t=0}^{\infty}$.

Let $\widetilde{V}$ denote the covariance matrix of inflation and housing price surprises, as defined in equation (98), implied by the locally unique equilibrium outcome for $\left\{\pi_{t}, y_{t}^{g a p}, \widehat{q}_{t}^{u}\right\}_{t=0}^{\infty}$ in lemma 3 and let $\Phi(V)=\widetilde{V}$ denote the function mapping the assumed covariance matrix $V$ into the realized covariance matrix $\widetilde{V}$ for inflation and housing price surprises, when belief distortions are given by the upper-bound distortions $\left\{\zeta_{t}^{* \pi}, \zeta_{t}^{* q}\right\}_{t=0}^{\infty}$. For the upper-bound belief distortions $\left\{\zeta_{t}^{* \pi}, \zeta_{t}^{* q}\right\}_{t=0}^{\infty}$ and the associated covariance matrix $V^{*}$, we have $\Phi\left(V^{*}\right)=V^{*}$.

Suppose that

$$
\partial v e c \Phi(V) / \partial v e c V \text { has rank } 3 \text {, }
$$

when evaluated at $V=V^{*}$. It then follows from the implicit function theorem that there is a locally unique fixed point $\Phi(V)=V$ for all belief distortions $\left\{\zeta_{t}^{\pi}, \zeta_{t}^{q}\right\}_{t=0}^{\infty}$ in the neighborhood of the upper bound belief distortions $\left\{\zeta_{t}^{* \pi}, \zeta_{t}^{* q}\right\}_{t=0}^{\infty}$. From lemma 3 we then obtain that there is a locally unique equilibrium outcome for $\left\{\pi_{t}, y_{t}^{g a p}, \widehat{q}_{t}^{u}\right\}_{t=0}^{\infty}$ associated with these belief distortions, as claimed in the main text. Condition (157) can verified numerically.

It remains to prove lemma 3. Consider a given process for belief distortions $\left\{\zeta_{t}^{\pi}, \zeta_{t}^{q}\right\}_{t=0}^{\infty}$ and a given matrix $V$. We can then uniquely solve equation (102) for a bounded linear process $\left\{\widehat{q}_{t}^{u}\right\}_{t=0}^{\infty}$. Taking expectations of the structural equations (101) and (102) delivers

$$
\begin{aligned}
E_{t} \pi_{t+1} & =\kappa_{y} E_{t} y_{t+1}^{g a p}+\beta E_{t} \pi_{t+2}+v_{t} \\
\Lambda_{\pi} E_{t} \pi_{t+1} & =-\frac{\Lambda_{y}}{\kappa_{y}}\left(E_{t} y_{t+1}^{g a p}-y_{t}^{g a p}\right)
\end{aligned}
$$


for all $t \geq 0$, where

$$
v_{t} \equiv E_{t} u_{t+1}+\kappa_{q} E_{t}\left(\widehat{q}_{t+1}^{u}-\widehat{q}_{t+1}^{u *}\right)+\beta \theta^{-1} V_{1}\left(E_{t} \zeta_{t+1}^{\pi}, E_{t} \zeta_{t+1}^{q}\right)^{\prime}
$$

is a known linear process. The system consisting of equations (158) and (159) has a unique bounded linear solution for the processes $\left\{E_{t} \pi_{t+1}, E_{t} y_{t+1}^{g a p}\right\}_{t=0}^{\infty}$ given the exogenous disturbance processes, linear processes for the belief distortions, and a value for $y_{0}^{g a p}$. Note that the system is the same one that determines the equilibrium evolution of expected inflation and the expected output gap under rational expectations and a commitment to the RE target criterion (76), except that the forcing term $v_{t}$ is now not simply given by $E_{t} u_{t+1} \cdot{ }^{31}$ In addition, the solutions for $\pi_{0}$ and $y_{0}^{g a p}$ must satisfy

$$
\begin{gathered}
\pi_{0}=\beta E_{0} \pi_{1}+\kappa_{y} y_{0}^{g a p}+\kappa_{q}\left(\widehat{q}_{0}^{u}-\widehat{q}_{0}^{u *}\right)+u_{0} \\
+\beta \theta^{-1} V_{1}\left(\zeta_{0}^{\pi}, \zeta_{0}^{q}\right)^{\prime}
\end{gathered}
$$

and the initial precommitment; these latter two linear conditions can be solved uniquely for $\pi_{0}$ and $y_{0}^{g a p}$. (Given our solution for $\widehat{q}_{0}^{u}$, the initial precommitment uniquely determines $\pi_{0}$; condition (160) then uniquely determines $y_{0}^{g a p}$ ). In this way, the forecastable components of the processes $\left\{\pi_{t}, y_{t}^{g a p}\right\}_{t=0}^{\infty}$ are uniquely determined given the matrix $V$ and the belief distortion processes $\left\{\zeta_{t}^{\pi}, \zeta_{t}^{q}\right\}_{t=0}^{\infty}$.

The time-invariant coefficients $\left(\pi_{0, k}, y_{0, k}^{g a p}\right)$ defining the surprise components of these processes must satisfy

$$
\begin{aligned}
\pi_{0, k} & =\kappa_{y} y_{0, k}^{g a p}+\kappa_{q}\left(q_{0, k}-q_{0, k}^{*}\right)+u_{0, k}+\beta \pi_{1, k}+\beta \theta^{-1} V_{1}\left(\zeta_{0, k}^{\pi}, \zeta_{0, k}^{q}\right)^{\prime} \\
\left(\Lambda_{\pi}+\theta^{-1} E_{\varphi \varphi}\right) \pi_{0, k} & =-\frac{\Lambda_{y}}{\kappa_{y}} y_{0, k}^{g a p}-(1-\delta) \theta^{-1} E_{\varphi \psi} q_{0, k},
\end{aligned}
$$

where the $\pi_{1, k}$ coefficients are given by the linear solution above for the process $\left\{E_{t} \pi_{t+1}\right\}_{t=0}^{\infty}$. Because

$$
\operatorname{det}\left(\begin{array}{cc}
1 & -\kappa_{y} \\
\Lambda_{\pi}+\theta^{-1} E_{\varphi \varphi} & \frac{\Lambda_{y}}{\kappa_{y}}
\end{array}\right)=\frac{\Lambda_{y}}{\kappa_{y}}+\kappa_{y}\left(\Lambda_{\pi}+\theta^{-1} E_{\varphi \varphi}\right)>0,
$$

the system (161)-(162) can be uniquely solved for the coefficients $\left(\pi_{0, k}, y_{0, k}^{g a p}\right)$ for each $k$. We have thus uniquely determined all of the coefficients describing linear processes for the endogenous variables $\left\{\pi_{t}, y_{t}^{g a p}, \widehat{q}_{t}^{u}\right\}_{t=0}^{\infty}$ implied by the belief distortions and the assumed matrix $V$.

\section{A.11 Details of the Model Parameterization}

Following table 5.1 in Woodford (2003), we set $\beta=0.99$ and $\kappa_{y}=0.024$. From Woodford's table 6.1 we take $\frac{\Lambda_{Y}}{\Lambda_{\tau}}=\lambda_{x}=0.048 / 16=0.0031$, where we divide by 16 because inflation rates in our model are expressed in quarterly rates of increase. We set the housing depreciation rate $\delta$ equal to $3 \%$ per year, following Adam, Marcet and Kuang (2012). We also have

$$
\begin{aligned}
& \kappa_{q}=-\frac{(1-\alpha \beta)(1-\alpha)}{\alpha(1+\eta \omega)} f_{q} \\
& \kappa_{y}=\frac{(1-\alpha \beta)(1-\alpha)}{\alpha(1+\eta \omega)}\left(k_{y}-f_{y}\right),
\end{aligned}
$$

where $f_{q}=\partial \log f / \partial \log q, k_{y}=\partial \log k / \partial \log y$ and $f_{y}=\partial \log f / \partial \log y$, so that

$$
\frac{\kappa_{q}}{\kappa_{y}}=-\frac{f_{q}}{k_{y}-f_{y}},
$$

\footnotetext{
${ }^{31}$ We have noted earlier that the system consisting of equations (75) and (76) has a unique bounded solution, given that $\kappa_{y}, \Lambda_{\pi}, \Lambda_{y}>0$ and $\beta \in(0,1)$.
} 
with $k_{y}-f_{y}$ denoting the elasticity of real marginal costs with respect to output. Following table 5.1 in Woodford (2003), we set $k_{y}-f_{y}=\varepsilon_{m c}=0.63$, so that we only need to determine $f_{q}$ in order to obtain a value for $\kappa_{q}$. Using

$$
f_{q}=\frac{(1+\chi) \overline{\Omega C}^{\chi}}{1+(1+\chi) \overline{\Omega C}^{\chi}}
$$

and assuming $\chi=\frac{\widetilde{\sigma}^{-1}}{1-\alpha}-1 \approx 0$, which for a long-run elasticity of housing supply equal to five $(\widetilde{\alpha}=0.8)$, in line with the estimated value in Adam, Kuang and Marcet (2012) and in the range of estimates in Topel and Rosen (1988), implies $\widetilde{\sigma}^{-1}=\frac{1}{5}$, we get

$$
f_{q} \approx \frac{\overline{\Omega C}^{\chi}}{1+\overline{\Omega C}^{\chi}}
$$

From

$$
\bar{Y}(1-\bar{g})=\bar{C}\left(1+\overline{\Omega C}^{\chi}\right)
$$

we can see that privately consumed output $\bar{Y}(1-\bar{g})$ is divided up into consumption $\bar{C}$ and resources invested in the housing sector, $\overline{\Omega C}^{1+\chi}$, so that $\overline{\Omega C}^{\chi}$ is the steady state ratio of housing investment over private expenditures. Over the period 1947-2012, this ratio, when measured as residential fixed investment spending over the sum of personal consumption expenditure and nonresidential fixed investment, is equal to $6.3 \%$ on average for the United States. ${ }^{32}$ We thus have

$$
f_{q} \approx 0.063 / 1.063 \approx 6 \%
$$

and obtain

$$
\kappa_{q}=-\frac{f_{q}}{k_{y}-f_{y}} \kappa_{y}=-\frac{0.06}{0.63} 0.024=-0.0023
$$

We also have ${ }^{33}$

$$
\frac{\Lambda_{q}}{\Lambda_{\pi}}=\frac{1}{1-\widetilde{\alpha}} \frac{\overline{\Omega C}^{\chi}}{1+\overline{\Omega C}^{\chi}} \frac{1}{\left(1+(1+\chi) \overline{\Omega C}^{\chi}\right)} \frac{1}{\varepsilon_{m c}} \frac{\Lambda_{Y}}{\Lambda_{\pi}} .
$$

Using the parameterization from above, then delivers

$$
\begin{aligned}
\frac{\Lambda_{q}}{\Lambda_{\pi}} & =\frac{1}{1-0.8} \frac{0.063}{1.063} \frac{1}{(1+0.063)} \frac{1}{0.63} 0.0031 \\
& \approx 0.0014 .
\end{aligned}
$$

Next, we determine the parameters of the stochastic process for $\widehat{\bar{\xi}}_{t}^{d}$. For this purpose we compute the log of the ratio of the nominal housing price index over nominal GDP for the US from 1975:Q1-2013:Q4 and extract a linear trend. The log deviations from trend have a quarterly autocorrelation of 0.9875 and a standard deviation of 0.072 . Assuming that about one quarter of those fluctuations reflect efficient fluctuations and that the sample autocorrelation slightly underestimates the actual auto-correlation at values close to one, we set $\rho_{\xi}=0.99$ and $\sigma_{e} \xi=0.024$. Assuming a $15 \%$ output subsidy for housing $\left(s^{d}=0.15\right)$, this determines the stochastic process for $\widehat{q}_{t}^{u *}$.

\footnotetext{
${ }^{32}$ We dowloaded data from FRED St Louis Fed, using the series PRFIA (Private Residential Fixed Investment, Billions of Dollars, Annual, Not Seasonally Adjusted), PCECA (Personal Consumption Expenditures, Billions of Dollars, Annual, Not Seasonally Adjusted), and PNFIA (Private Nonresidential Fixed Investment, Billions of Dollars, Annual, Not Seasonally Adjusted).

${ }^{33}$ This follows from calculations that are contained in our notes "AW notes 091415", which are available upon request.
} 
Finally, we determine the mark-up distortions. To compute the steady state mark-up distortion $w$, note that $\kappa_{y}=0.024, k_{y}-f_{y}=0.63$, and equations (163) and (164) jointly imply

$$
\frac{(1-\alpha)(1-\alpha \beta)}{\alpha(1+\omega \eta)}=\frac{0.024}{0.63} .
$$

Assuming $\Theta=0.15$, which means that the output subsidy falls short $15 \%$ of its optimal steady state value, we get

$$
\begin{aligned}
w & =\frac{(1-\alpha)(1-\alpha \beta)}{\alpha(1+\omega \eta)} \Theta \\
& =\frac{0.024}{0.63} 0.15 \\
& \approx 0.0057 .
\end{aligned}
$$

From the mark-up shock estimates for the post-1980 sample reported in table 3 in Ireland (2004), we obtain $\rho_{u}=0.9907$ and $\sigma_{e^{u}}=0.0002$. 


\section{ONLINE APPENDIX - NOT FOR PUBLICATION}

"Robustly Optimal Monetary Policy in a New Keynesian Model with Housing" Klaus Adam and Michael Woodford

\section{B Numerically Solving for the Upper Bound Dynamics}

\section{B.1 The Upper Bound Dynamics}

From equations (113)-(117) in the main text we have:

$$
\begin{aligned}
& \Lambda_{\pi} \pi_{t}-\varphi_{t}+\varphi_{t-1}+\theta^{-1} E_{\varphi \varphi}\left(\pi_{t}-E_{t-1} \pi_{t}\right) \\
&+\theta^{-1}(1-\delta) E_{\varphi \psi}\left(\widehat{q}_{t}^{u}-E_{t-1} \widehat{q}_{t}^{u}\right)=0 \\
& \Lambda_{Y} y_{t}^{g a p}+\kappa_{y} \varphi_{t}=0 \\
& \Lambda_{q}\left(\widehat{q}_{t}^{u}-\widehat{q}_{t}^{u *}\right)+\kappa_{q} \varphi_{t}-\psi_{t}+(1-\delta) \psi_{t-1} \\
&+\theta^{-1}(1-\delta) E_{\varphi \psi}\left(\pi_{t}-E_{t-1} \pi_{t}\right) \\
&+\theta^{-1}(1-\delta)^{2} E_{\psi \psi}\left(\widehat{q}_{t}^{u}-E_{t-1} \widehat{q}_{t}^{u}\right)=0 \\
& \pi_{t}-\kappa_{y} y_{t}^{g a p}-\kappa_{q}\left(\widehat{q}_{t}^{u}-\widehat{q}_{t}^{u *}\right)-\beta E_{t} \pi_{t+1}-u_{t} \\
&-\theta^{-1} \beta \varphi_{t} E\left[\left(\pi_{t+1}-E_{t} \pi_{t+1}\right)^{2}\right] \\
&-\theta^{-1} \beta(1-\delta) \psi_{t} E\left[\left(\pi_{t+1}-E_{t} \pi_{t+1}\right)\left(\widehat{q}_{t+1}^{u}-E_{t} \widehat{q}_{t+1}^{u}\right)\right]=0 \\
& \widehat{q}_{t}^{u}-(1-\beta(1-\delta)) \widehat{\xi}_{t}^{d}-\beta(1-\delta) E_{t} \widehat{q}_{t+1}^{u} \\
&-\theta^{-1} \beta(1-\delta) \varphi_{t} E\left[\left(\pi_{t+1}-E_{t} \pi_{t+1}\right)\left(\widehat{q}_{t+1}^{u}-E_{t} \widehat{q}_{t+1}^{u}\right)\right] \\
&-\theta^{-1} \beta(1-\delta)^{2} \psi_{t} E\left[\left(\widehat{q}_{t+1}^{u}-E_{t} \widehat{q}_{t+1}^{u}\right)^{2}\right]=0,
\end{aligned}
$$

where $E_{\varphi \varphi}, E_{\varphi \psi}$ and $E_{\psi \psi}$ are defined in equation (121), and where $u_{t}$ is defined in (77). Log-linearizing equation (72) around the optimal steady state (in which there are no steady state distortions), we get

$$
\widehat{q}_{t}^{u *}=\widehat{\bar{\xi}}_{t}^{d}-s^{d}
$$

where $s^{d} \sim O(\sigma)$ and $\widehat{q}_{t}^{u *}$ denotes the log deviation of $q_{t}^{u *}$ from the optimal steady state. The previous equation has been used to derive equation (169) from equation (117).

We wish to solve (165)-(169) for the equilibrium values of $\left\{\pi_{t}, y_{t}^{g a p}, \widehat{q}_{t}^{u}, \varphi_{t}, \psi_{t}\right\}_{t=0}^{\infty}$, given the exogenous processes $\left\{\widehat{u}_{t}, \widehat{\bar{\xi}}\right\}_{t=0}^{\infty}$ and the initial conditions $\left(\varphi_{-1}, \psi_{-1}\right)$.

\section{B.2 Parameterization Approach}

Let us define

$$
\begin{aligned}
\left(\theta^{\text {new }}\right)^{-1} & \equiv(\theta)^{-1} / \Lambda_{\pi} \\
\varphi_{t}^{\text {new }} & \equiv \varphi_{t} / \Lambda_{\pi} \\
\psi_{t}^{\text {new }} & \equiv \psi_{t} / \Lambda_{\pi}
\end{aligned}
$$

and

$$
u_{t} \equiv w+\widehat{u}_{t}
$$


where

$$
\begin{aligned}
w & \equiv \frac{(1-\alpha)(1-\alpha \beta)}{\alpha(1+\omega \eta)} \Theta \\
\widehat{u}_{t} & \equiv \frac{(1-\alpha)(1-\alpha \beta)}{\alpha(1+\omega \eta)}\left(\widehat{\tau}_{t}-\widehat{g}_{t}\right) .
\end{aligned}
$$

We also define

$$
\begin{aligned}
& E_{\varphi \varphi}^{\text {new }} \equiv(1-\beta) E_{0} \sum_{t=0}^{\infty} \beta^{t}\left(\varphi_{t}^{\text {new }}\right)^{2}>0 \\
& E_{\varphi \psi}^{\text {new }} \equiv(1-\beta) E_{0} \sum_{t=0}^{\infty} \beta^{t} \varphi_{t}^{\text {new }} \psi_{t}^{\text {new }} \\
& E_{\psi \psi}^{\text {new }} \equiv(1-\beta) E_{0} \sum_{t=0}^{\infty} \beta^{t}\left(\psi_{t}^{\text {new }}\right)^{2}>0 .
\end{aligned}
$$

We can then write (165)-(169) as

$$
\begin{aligned}
& \pi_{t}-\varphi_{t}^{\text {new }}+\varphi_{t-1}^{\text {new }}+\left(\theta^{\text {new }}\right)^{-1} E_{\varphi \varphi}^{\text {new }}\left(\pi_{t}-E_{t-1} \pi_{t}\right) \\
& +\left(\theta^{\text {new }}\right)^{-1}(1-\delta) E_{\varphi \psi}^{\text {new }}\left(\widehat{q}_{t}^{u}-E_{t-1} \widehat{q}_{t}^{u}\right)=0 \\
& \frac{\Lambda_{Y}}{\Lambda_{\pi}} y_{t}^{\text {gap }}+\kappa_{y} \varphi_{t}^{\text {new }}=0 \\
& \frac{\Lambda_{q}}{\Lambda_{\pi}}\left(\widehat{q}_{t}^{u}-\widehat{q}_{t}^{u *}\right)+\kappa_{q} \varphi_{t}^{\text {new }}-\psi_{t}^{\text {new }}+(1-\delta) \psi_{t-1}^{\text {new }} \\
& +\left(\theta^{\text {new }}\right)^{-1}(1-\delta) E_{\varphi \psi}^{\text {new }}\left(\pi_{t}-E_{t-1} \pi_{t}\right) \\
& +\left(\theta^{\text {new }}\right)^{-1}(1-\delta)^{2} E_{\psi \psi}^{\text {new }}\left(\widehat{q}_{t}^{u}-E_{t-1} \widehat{q}_{t}^{u}\right)=0 \\
& \pi_{t}-\kappa_{y} y_{t}^{g a p}-\kappa_{q}\left(\widehat{q}_{t}^{u}-\widehat{q}_{t}^{u *}\right)-\beta E_{t} \pi_{t+1}-w-\widehat{u}_{t} \\
& -\left(\theta^{\text {new }}\right)^{-1} \beta \varphi_{t}^{\text {new }} E\left[\left(\pi_{t+1}-E_{t} \pi_{t+1}\right)^{2}\right] \\
& -\left(\theta^{\text {new }}\right)^{-1} \beta(1-\delta) \psi_{t}^{\text {new }} E\left[\left(\pi_{t+1}-E_{t} \pi_{t+1}\right)\left(\widehat{q}_{t+1}^{u}-E_{t} \widehat{q}_{t+1}^{u}\right)\right]=0 \\
& \widehat{q}_{t}^{u}-(1-\beta(1-\delta)) \widehat{\xi}_{t}^{d}-\beta(1-\delta) E_{t} \widehat{q}_{t+1}^{u} \\
& -\left(\theta^{\text {new }}\right)^{-1} \beta(1-\delta) \varphi_{t}^{\text {new }} E\left[\left(\pi_{t+1}-E_{t} \pi_{t+1}\right)\left(\widehat{q}_{t+1}^{u}-E_{t} \widehat{q}_{t+1}^{u}\right)\right] \\
& -\left(\theta^{\text {new }}\right)^{-1} \beta(1-\delta)^{2} \psi_{t}^{\text {new }} E\left[\left(\widehat{q}_{t+1}^{u}-E_{t} \widehat{q}_{t+1}^{u}\right)^{2}\right]=0 .
\end{aligned}
$$

The previous equations show that the upper bound solution $<$ is determined by the parameters $\beta, \delta, \kappa_{y}, \kappa_{q}, \frac{\Lambda_{Y}}{\Lambda_{\pi}}, \frac{\Lambda_{q}}{\Lambda_{\pi}}, s^{d}, w$ and $\left(\theta^{\text {new }}\right)^{-1}$, as well as stochastic processes for $\widehat{u}_{t}$ and $\widehat{\bar{\xi}}_{t}^{d}$ (the latter determines $\widehat{\xi}_{t}^{d}$ and jointly with $s^{d}$ also $\left.\widehat{q}_{t}^{u *}\right)$. The model is solved for the parameters reported in table 1 of the main text.

\section{B.3 Solution Approach}

We simplify (176)-(180) by using (177) to eliminate the output gap:

$$
y_{t}^{g a p}=-\frac{\kappa_{y}}{\Lambda_{Y} / \Lambda_{\pi}} \varphi_{t}^{\text {new }}
$$

We furthermore simplify using $\widehat{q}_{t}^{u *}=\widehat{\bar{\xi}}_{t}^{d}-s^{d}$ and the relationship

$$
\widehat{\bar{\xi}}_{t}^{d}=(1-(1-\delta) \beta) \widehat{\xi}_{t}^{d}+(1-\delta) \beta E_{t} \widehat{\bar{\xi}}_{t+1}^{d},
$$


together with the law of motion for $\widehat{\bar{\xi}}_{t}^{d}$ to express $\widehat{\xi}_{t}^{d}$ as a function of $\widehat{\bar{\xi}}_{t}^{d}$ :

$$
\widehat{\xi}_{t}^{d}=\frac{1-(1-\delta) \beta \rho_{\xi}}{1-(1-\delta) \beta} \overline{\bar{\xi}}_{t}^{d}
$$

This delivers

$$
\begin{gathered}
\pi_{t}-\varphi_{t}^{\text {new }}+\varphi_{t-1}^{\text {new }}+\left(\theta^{\text {new }}\right)^{-1} E_{\varphi \varphi}^{\text {new }}\left(\pi_{t}-E_{t-1} \pi_{t}\right) \\
+\left(\theta^{\text {new }}\right)^{-1}(1-\delta) E_{\varphi \psi}^{\text {new }}\left(\widehat{q}_{t}^{u}-E_{t-1} \widehat{q}_{t}^{u}\right)=0 \\
\frac{\Lambda_{q}}{\Lambda_{\pi}}\left(\widehat{q}_{t}^{u}-\widehat{\bar{\xi}}_{t}^{d}-s^{d}\right)+\kappa_{q} \varphi_{t}^{\text {new }}-\psi_{t}^{\text {new }}+(1-\delta) \psi_{t-1}^{\text {new }} \\
+\left(\theta^{\text {new }}\right)^{-1}(1-\delta) E_{\varphi \psi}^{\text {new }}\left(\pi_{t}-E_{t-1} \pi_{t}\right) \\
+\left(\theta^{\text {new }}\right)^{-1}(1-\delta)^{2} E_{\psi \psi}^{\text {new }}\left(\widehat{q}_{t}^{u}-E_{t-1} \widehat{q}_{t}^{u}\right)=0 \\
\pi_{t}+\frac{\left(\kappa_{y}\right)^{2}}{\Lambda_{Y} / \Lambda_{\pi}} \varphi_{t}^{\text {new }}-\kappa_{q} \widehat{q}_{t}^{u}+\kappa_{q} \widehat{\bar{\xi}}_{t}^{d}-\kappa_{q} s^{d}-\beta E_{t} \pi_{t+1}-w-\widehat{u}_{t} \\
-\left(\theta^{\text {new }}\right)^{-1} \beta \varphi_{t}^{n e w} E\left[\left(\pi_{t+1}-E_{t} \pi_{t+1}\right)^{2}\right] \\
-\left(\theta^{\text {new }}\right)^{-1} \beta(1-\delta) \psi_{t}^{\text {new }} E\left[\left(\pi_{t+1}-E_{t} \pi_{t+1}\right)\left(\widehat{q}_{t+1}^{u}-E_{t} \widehat{q}_{t+1}^{u}\right)\right]=0 \\
\widehat{q}_{t}^{u}-\left(1-(1-\delta) \beta \rho_{\xi}\right) \widehat{\bar{\xi}}_{t}^{d}-\beta(1-\delta) E_{t} \widehat{q}_{t+1}^{u} \\
-\left(\theta^{\text {new }}\right)^{-1} \beta(1-\delta) \varphi_{t}^{\text {new }} E\left[\left(\pi_{t+1}-E_{t} \pi_{t+1}\right)\left(\widehat{q}_{t+1}^{u}-E_{t} \widehat{q}_{t+1}^{u}\right)\right] \\
-\left(\theta^{\text {new }}\right)^{-1} \beta(1-\delta)^{2} \psi_{t}^{\text {new }} E\left[\left(\widehat{q}_{t+1}^{u}-E_{t} \widehat{q}_{t+1}^{u}\right)^{2}\right]=0 .
\end{gathered}
$$

We look for time-invariant solutions to these equations in which the variables

$$
x_{t}^{\prime}=\left(\varphi_{t}^{\text {new }}, \psi_{t}^{\text {new }}, \pi_{t}, \widehat{q}_{t}^{u}, \widehat{\bar{\xi}}_{t}^{d}, \widehat{u}_{t}, E_{t} \pi_{t+1}, E_{t} \widehat{q}_{t+1}^{u}\right)
$$

evolve as follows

$$
x_{t}=A+B x_{t-1}+C\left(\begin{array}{c}
e_{t}^{\xi} \\
e_{t}^{u}
\end{array}\right)
$$

The conjectured solution structure (188) is self-consistent in the following sense: given a conjecture about the solution parameters $P \equiv(A, B, C)$ and given initial conditions $\left(x_{-1}, \widehat{u}_{0,}, \widehat{\bar{\xi}}_{0}^{d}\right)$, one can determine the coefficients $E_{\varphi \varphi}^{n e w}, E_{\varphi \psi}^{n e w}$ and $E_{\psi \psi}^{\text {new }}$ defined in (173)-(175). In addition, one can determine the time-invariant values

$$
\begin{aligned}
E_{\pi \pi} & =E_{t}\left[\left(\pi_{t+1}-E_{t} \pi_{t+1}\right)^{2}\right] \\
E_{\pi q} & =E_{t}\left[\left(\pi_{t+1}-E_{t} \pi_{t+1}\right)\left(\widehat{q}_{t+1}^{u}-E_{t} \widehat{q}_{t+1}^{u}\right)\right] \\
E_{q q} & =E_{t}\left[\left(\widehat{q}_{t+1}^{u}-E_{t} \widehat{q}_{t+1}^{u}\right)^{2}\right] .
\end{aligned}
$$

Given the conjectured solution structure (188), equation system (184)-(187) is thus a linear expectational difference equation system with time invariant coefficients of the form

$$
\Gamma_{0} x_{t}=\Gamma_{c}+\Gamma_{1} x_{t-1}+\Gamma_{\Psi}\left(\begin{array}{c}
e_{t}^{\xi} \\
e_{t}^{u}
\end{array}\right)+\Gamma_{\Pi}\left(\begin{array}{c}
\eta_{t}^{\pi} \\
\eta_{t}^{q}
\end{array}\right)
$$


where $\eta_{t}^{\pi}=\pi_{t}-E_{t-1} \pi_{t}$ and $\eta_{t}^{q}=\widehat{q}_{t}^{u}-E_{t-1} \widehat{q}_{t}^{u}$ denote forecast errors. Equation (189) has a solution of the form (188), which can be readily computed using the approach developed in Sims (2000). Under the conjecture (188), the only non-standard feature of equations (184)$(187)$ is that the coefficients $\widetilde{\mathcal{E}}=\left(E_{\varphi \varphi}^{n e w}, E_{\varphi \psi}^{\text {new }}, E_{\psi \psi}^{\text {new }}, E_{\pi \pi}, E_{\pi q}, E_{q q}\right)$ entering the system depend themselves on the solution. Solving the equation system thus requires solving a fixed point problem.

We now explain how we solve for the fixed point. In a first step, we start by solving (184)(187) for the case $\left(\theta^{\text {new }}\right)^{-1}=0$, i.e., for the case without robustness concerns. Equations (184)(187) are then independent of $\widetilde{\mathcal{E}}$ and assume the standard form (189) with known coefficients. The REE solution serves as a benchmark to which we can compare the outcomes for the case with robustness concerns and we use the set of coefficient $\widetilde{\mathcal{E}}$ implied by it as a starting guess when solving for the case with robustness concerns.

Given some guess $\widetilde{\mathcal{E}}$, the coefficients in (189) implied by (184)-(187) are given as follows:

$\Gamma_{0}=$

$\left(\begin{array}{cccccccc}-1 & 0 & 1 & 0 & 0 & 0 & 0 & 0 \\ \kappa_{q} & -1 & 0 & \frac{\Lambda_{q}}{\Lambda_{\pi}} & -\frac{\Lambda_{q}}{\Lambda_{\pi}} & 0 & 0 & 0 \\ \frac{\left(\kappa_{y}\right)^{2}}{\Lambda_{Y} / \Lambda_{\pi}}-\left(\theta^{\text {new }}\right)^{-1} \beta E_{\pi \pi} & -\left(\theta^{\text {new }}\right)^{-1} \beta(1-\delta) E_{\pi q} & 1 & -\kappa_{q} & \kappa_{q} & -1 & -\beta & 0 \\ -\left(\theta^{\text {new }}\right)^{-1} \beta(1-\delta) E_{\pi q} & -\left(\theta^{\text {new }}\right)^{-1} \beta(1-\delta)^{2} E_{q q} & 0 & 1 & -\left(1-(1-\delta) \beta \rho_{\xi}\right) & 0 & 0 & -\beta(1-\delta) \\ 0 & 0 & 0 & 0 & 1 & 0 & 0 & 0 \\ 0 & 0 & 0 & 0 & 0 & 1 & 0 & 0 \\ 0 & 0 & 1 & 0 & 0 & 0 & 0 & 0 \\ 0 & 0 & 0 & 1 & 0 & 0 & 0 & 0\end{array}\right.$




$$
\begin{aligned}
& \Gamma_{c}^{\prime}=\left(\begin{array}{llllllll}
0 & -\frac{\Lambda_{q}}{\Lambda_{\pi}} s^{d} & \omega+\kappa_{q} s^{d} & 0 & 0 & 0 & 0 & 0
\end{array}\right) \\
& \Gamma_{1}=\left(\begin{array}{cccccccc}
-1 & 0 & 0 & 0 & 0 & 0 & 0 & 0 \\
0 & -(1-\delta) & 0 & 0 & 0 & 0 & 0 & 0 \\
0 & 0 & 0 & 0 & 0 & 0 & 0 & 0 \\
0 & 0 & 0 & 0 & 0 & 0 & 0 & 0 \\
0 & 0 & 0 & 0 & \rho_{\xi} & 0 & 0 & 0 \\
0 & 0 & 0 & 0 & 0 & \rho_{u} & 0 & 0 \\
0 & 0 & 0 & 0 & 0 & 0 & 1 & 0 \\
0 & 0 & 0 & 0 & 0 & 0 & 0 & 1
\end{array}\right) \\
& \Gamma_{\Psi}=\left(\begin{array}{ll}
0 & 0 \\
0 & 0 \\
0 & 0 \\
0 & 0 \\
1 & 0 \\
0 & 1 \\
0 & 0 \\
0 & 0
\end{array}\right) \\
& \Gamma_{\Pi}=\left(\begin{array}{cc}
-\left(\theta^{\text {new }}\right)^{-1} E_{\varphi \varphi}^{\text {new }} & -\left(\theta^{\text {new }}\right)^{-1}(1-\delta) E_{\varphi \psi}^{\text {new }} \\
-\left(\theta^{\text {new }}\right)^{-1}(1-\delta) E_{\varphi \psi}^{\text {new }} & -\left(\theta^{\text {new }}\right)^{-1}(1-\delta)^{2} E_{\psi \psi}^{\text {new }} \\
0 & 0 \\
0 & 0 \\
0 & 0 \\
0 & 0 \\
1 & 0 \\
0 & 1
\end{array}\right)
\end{aligned}
$$

Solving (189) with these coefficients delivers a set of solution coefficients $P(\widetilde{\mathcal{E}})=\{A(\widetilde{\mathcal{E}}), B(\widetilde{\mathcal{E}}), C(\widetilde{\mathcal{E}})\}$ for (188). The latter coefficients then imply, together with the initial values $\left(x_{-1}, \widehat{u}_{0}, \widehat{\bar{\xi}}_{0}\right)$, which we set equal to the steady state values implied by $P(\widetilde{\mathcal{E}})$, an implied value for $\widetilde{\mathcal{E}}$, which we denote $Z(\widetilde{\mathcal{E}})$. We then solve for the value $\widetilde{\mathcal{E}}^{*}$ satisfying

$$
\widetilde{\mathcal{E}}^{*}=Z\left(\widetilde{\mathcal{E}}^{*}\right)
$$

using a standard root finding routine. The implied values for the components $E_{\pi \pi}, E_{\pi q}, E_{q q}$ in $Z(\widetilde{\mathcal{E}})$ can be readily computed from the solution coefficients $P(\widetilde{\mathcal{E}})$. The updated guesses for $E_{\varphi \varphi}^{n e w}, E_{\varphi \psi}^{n e w}, E_{\psi \psi}^{n e w}$ can be computed using the following observations. The solution (188) implies for $t \geq 1$ and for a starting value $x_{0}$ at the steady state $^{34}$, the following recursive law of motion for $E_{0} x_{t} x_{t}^{\prime}$ :

$$
\begin{aligned}
E_{0}\left[x_{t} x_{t}^{\prime}\right] & =E_{0}\left[\left(A+B x_{t-1}+C e_{t}\right)\left(A^{\prime}+x_{t-1}^{\prime} B^{\prime}+e_{t}^{\prime} C^{\prime}\right)\right] \\
& =A A^{\prime}+A x_{0}^{\prime} B^{\prime}+B x_{0} A^{\prime}+C \Sigma_{e} C^{\prime} \\
& +B E_{0}\left[x_{t-1} x_{t-1}^{\prime}\right] B^{\prime}
\end{aligned}
$$

where $e_{t}^{\prime} \equiv\left(e_{t}^{\xi}, e_{t}^{u}\right)$ and $\Sigma_{e} \equiv V A R\left(e_{t}\right)$ and where we use the fact that for our initial conditions

\footnotetext{
${ }^{34}$ The fact that $x_{0}$ is at the steady state is implied by our assumptions about the initial conditions for $\left(x_{-1}, \widehat{u}_{0}, \widehat{\bar{\xi}}_{0}^{d}\right)$.
} 
$E_{0} x_{t}=x_{0}$. We start the iteration with

$$
\begin{aligned}
E_{0}\left[x_{1} x_{1}^{\prime}\right] & =A A^{\prime}+A x_{0}^{\prime} B^{\prime}+B x_{0} A^{\prime}+C \Sigma_{e} C^{\prime}+B x_{0} x_{0}^{\prime} B^{\prime} \\
& =\left(A+B x_{0}\right)\left(A^{\prime}+x_{0}^{\prime} B^{\prime}\right)+C \Sigma_{e} C^{\prime} \\
& =x_{0} x_{0}^{\prime}+C \Sigma_{e} C^{\prime} .
\end{aligned}
$$

Then

$$
\begin{aligned}
E_{0}\left[x_{2} x_{2}^{\prime}\right] & =A A^{\prime}+A x_{0}^{\prime} B^{\prime}+B x_{0} A^{\prime}+C \Sigma_{e} C^{\prime}+B E_{0}\left[x_{1} x_{1}^{\prime}\right] B^{\prime} \\
& =A A^{\prime}+A x_{0}^{\prime} B^{\prime}+B x_{0} A^{\prime}+C \Sigma_{e} C^{\prime}+B\left(x_{0} x_{0}^{\prime}+C \Sigma_{e} C^{\prime}\right) B^{\prime} \\
& =x_{0} x_{0}^{\prime}+C \Sigma_{e} C^{\prime}+B C \Sigma_{e} C^{\prime} B^{\prime}
\end{aligned}
$$

and more generally for $t \geq 0$

$$
E_{0} x_{t} x_{t}^{\prime}=x_{0} x_{0}^{\prime}+\sum_{j=1}^{t} B^{j-1} C \Sigma_{e} C^{\prime}\left(B^{\prime}\right)^{j-1},
$$

so that

$$
\begin{aligned}
(1-\beta) \sum_{t=0}^{\infty} \beta^{t} E_{0} x_{t} x_{t}^{\prime} & =x_{0} x_{0}^{\prime}+(1-\beta) \sum_{t=1}^{\infty} \beta^{t} \sum_{j=1}^{t} B^{j-1} C \Sigma_{e} C^{\prime}\left(B^{\prime}\right)^{j-1} \\
& =x_{0} x_{0}^{\prime}+\sum_{j=1}^{t} \beta^{j} B^{j-1} C \Sigma_{e} C^{\prime}\left(B^{\prime}\right)^{j-1} .
\end{aligned}
$$

We numerically compute the sum in the last row by truncating the infinite sequence when the terms $\beta^{j} B^{j-1} C \Sigma_{e} C^{\prime}\left(B^{\prime}\right)^{j-1}$ reach values below $\left(1-\beta \lambda_{B}\right) \cdot 10^{-9}$ where $\lambda_{B}<1$ is the largest eigenvalue of $B$. This insures that approximation errors from computing the truncated sum are below $10^{-9}$. The values for $E_{\varphi \varphi}^{n e w}, E_{\varphi \psi}^{n e w}, E_{\psi \psi}^{n e w}$ are then given by elements $(1,1),(1,2)$ and $(2,2)$, respectively, of the matrix $(1-\beta) \sum_{t=0}^{\infty} \beta^{t} E_{0} x_{t} x_{t}^{\prime}$.

The numerical code also checks whether the matrix $H$ in equation (122) is positive semidefinite, which insures that the policymaker's best response in fact achieves a minimum loss in the upper bound solution. Since $H \equiv \Lambda_{\pi} \cdot H^{\text {new }}$, with

$$
H^{\text {new }} \equiv \frac{1}{2}\left(\begin{array}{cc}
1+\left(\theta^{\text {new }}\right)^{-1} E_{\varphi \varphi}^{\text {new }} & \left(\theta^{\text {new }}\right)^{-1}(1-\delta) E_{\varphi \psi}^{\text {new }} \\
\left(\theta^{\text {new }}\right)^{-1}(1-\delta) E_{\varphi \psi}^{\text {new }} & \Lambda_{y} / \Lambda_{\pi}+\left(\theta^{\text {new }}\right)^{-1}(1-\delta)^{2} E_{\psi \psi}^{\text {new }}
\end{array}\right),
$$

this can be achieved by checking whether $H^{\text {new }}$ is positive semidefinite.

The MatLab routine 'Solve_UpperBound.m' reliably solves for the fixed point $\widetilde{\mathcal{E}}^{*}$ in $(190)$ for all values $\left(\theta^{\text {new }}\right)^{-1} \leq 54$ when using the parameters from table 1 in the main text. For values $\left(\theta^{\text {new }}\right)^{-1}>54$, one gets problems with non-existence of equilibria during the numerical fixed point search, i.e., the conjectured solution coefficients $\mathcal{E}$ imply that equation system (189) has no non-explosive solution. The Matlab routine computes the steady states and the impulse responses to shocks for the RE optimal policy and for the upper bound dynamics. Table 2 in the main text reports the steady state outcomes for $\left(\theta^{\text {new }}\right)^{-1}=50$.

\section{Verifying that the Targeting Rule Implements the Up- per Bound}

The MatLab routine 'Solve_UpperBound.m' also evaluates whether condition (157) in appendix A.10 is satisfied. This can be achieved by checking whether the matrix

$$
\frac{\partial\left(\widetilde{E}_{\pi \pi}, \widetilde{E}_{\pi q}, \widetilde{E}_{q q}\right)}{\partial\left(E_{\pi \pi}, E_{\pi q}, E_{q q}\right)^{\prime}} \text { has rank } 3
$$


when evaluated at the upper bound solution, where $\left(\widetilde{E}_{\pi \pi}, \widetilde{E}_{\pi q}, \widetilde{E}_{q q}\right)$ denotes the values of inflation and housing price surprises implied by the solution to equations (184)-(187) for the assumed values $\left(E_{\pi \pi}, E_{\pi q}, E_{q q}\right)$. It insures that there is locally a unique RE equilibrium for alternative belief distortions around the upper bound distortions.

We can now evaluate the outcomes associated with alternative belief distortions when the policymaker commits to the proposed targeting rule (123). We do so by considering values for $V$ in the neighborhood of the upper bound values $V^{*}$. We can then construct belief distortions by using the predictable dynamics involved by the solution to equations (184)-(187) for the considered value of $V$. In this solution the multipliers $\left(\varphi_{t}^{\text {new }}, \psi_{t}^{\text {new }}\right)$ can be written for $t \geq 1$ as linear functions of their lagged values, the lagged shock values $\left(\widehat{\bar{\xi}}_{t-1}^{d}, \widehat{u}_{t-1}\right)$, the lagged expectations $\left(E_{t-1} \pi_{t}, E_{t-1} \widehat{q}_{t}^{u}\right)$ and the shock innovations $\left(e_{t}^{\xi}, e_{t}^{u}\right)$. It then follows from $E_{t-1} \zeta_{t}^{\text {new }}=E_{t-1} \Lambda_{\Pi} \zeta_{t}=E_{t-1}\left(\varphi_{t}^{\text {new* }},(1-\delta) \psi_{t}^{*}\right)^{\prime}$ that the (scaled) worst-case belief distortions for $t \geq 1$ can similarly be written as

$$
\zeta_{t}^{\text {new }}=a_{0}+A_{0}\left(\begin{array}{c}
\zeta_{t-1}^{n e w} \\
\widehat{\bar{\xi}}_{t-1}^{d} \\
\widehat{u}_{t-1} \\
E_{t-1} \pi_{t} \\
E_{t-1} \widehat{q}_{t}^{u}
\end{array}\right)+A_{1}\left(\begin{array}{c}
e_{t}^{\xi} \\
e_{t}^{u}
\end{array}\right) .
$$

where the $2 \mathrm{x} 1$ vector $a_{0}$ the and the $2 \mathrm{x} 6$ matrix $A_{0}$ are implied by the predictable dynamics; the $2 \times 2$ matrix $A_{1}$ contains the still undetermined surprise coefficients. We consider surprise coefficients in the neighborhood of the values implied by belief distortions in the upper bound solution, such that they are consistent with the assumed matrix of surprises $V$. The latter requires solving a fixed point problem, as we now explain.

Given a value for $A_{1}$ and given the considered value $V$, we can determine the locally unique outcome $\left\{\zeta_{t}, \pi_{t}, y_{t}^{g a p}, \widehat{q}_{t}^{u}\right\}_{t=1}^{\infty}$ using the structural equations (101) and (102) and the targeting rule (123). Using (170),(182), (183), and (171) these equations can be expressed as

$$
\begin{aligned}
0= & \kappa_{q} s^{d}+w-\pi_{t}+\beta E_{t} \pi_{t+1}+\kappa_{y} y_{t}^{\text {gap }}+\kappa_{q} \widehat{q}_{t}^{u}+\beta\left(\theta^{\text {new }}\right)^{-1} V_{1} \widehat{\zeta}_{t}^{\text {new }}-\kappa_{q} \widehat{\bar{\xi}}_{t}^{d}+\widehat{u}_{t} \\
0= & -\widehat{q}_{t}^{u}+\beta(1-\delta) E_{t}\left[\widehat{q}_{t+1}^{u}\right]+\beta(1-\delta)\left(\theta^{\text {new }}\right)^{-1} V_{2} \widehat{\zeta}_{t}^{\text {new }}+\left(1-\beta(1-\delta) \rho_{\xi}\right) \widehat{\bar{\xi}}_{t}^{d} \\
0= & \pi_{t}+\frac{\Lambda_{y}}{\Lambda_{\pi} \kappa_{y}}\left(y_{t}^{g a p}-y_{t-1}^{g a p}\right) \\
& +\left(\theta^{\text {new }}\right)^{-1} E_{\varphi \varphi}\left(\pi_{t}-E_{t-1} \pi_{t}\right)+\left(\theta^{\text {new }}\right)^{-1}(1-\delta) E_{\varphi \psi}\left(\widehat{q}_{t}^{u}-E_{t-1} \widehat{q}_{t}^{u}\right)
\end{aligned}
$$

For given $V$, equations (192)-(195) take the standard difference equation form (189) with $x_{t}=$ $\left(\pi_{t}, y_{t}^{g a p}, \widehat{q}_{t}^{u}, \zeta_{t}^{\text {new*1 }}, \widehat{\bar{\xi}}_{t}^{d}, \widehat{u}_{t}, E_{t} \pi_{t+1}, E_{t} \widehat{q}_{t+1}^{u}\right)^{\prime}$ and

$\Gamma_{0}=\left(\begin{array}{lllllllll}0 & 0 & 0 & 1 & 0 & 0 & 0 & 0 & 0 \\ 0 & 0 & 0 & 0 & 1 & 0 & 0 & 0 & 0 \\ 1 & -\kappa_{y} & -\kappa_{q} & -\beta\left(\theta^{\text {new }}\right)^{-1} V_{1} & \kappa_{q} & -1 & -\beta & 0 \\ 0 & 0 & 1 & -\beta(1-\delta)\left(\theta^{\text {new }}\right)^{-1} V_{2} & -\left(1-\beta(1-\delta) \rho_{\xi}\right) & 0 & 0 & -\beta(1-\delta) \\ -1 & -\frac{\Lambda_{y}}{\Lambda_{\pi} \kappa_{y}} & 0 & 0 & 0 & 0 & 0 & 0 & 0 \\ 0 & 0 & 0 & 0 & 0 & 1 & 0 & 0 & 0 \\ 0 & 0 & 0 & 0 & 0 & 0 & 1 & 0 & 0 \\ 1 & 0 & 0 & 0 & 0 & 0 & 0 & 0 & 0 \\ 0 & 0 & 1 & 0 & 0 & 0 & 0 & 0 & 0\end{array}\right)$ 


$$
\Gamma_{1}=\left(\begin{array}{ccccc}
a_{0} \\
\kappa_{q} s^{d}+w \\
0 \\
0 \\
\end{array}\right.
$$

where $A_{0, i}$ denotes the $i$-th row of $A_{0}$, and

$$
\Gamma_{\Psi}=\left(\begin{array}{cc}
A_{1,1} \\
A_{1,2} \\
0 & 0 \\
0 & 0 \\
0 & 0 \\
1 & 0 \\
0 & 1 \\
0 & 0 \\
0 & 0
\end{array}\right)
$$

where $A_{1, i}$ denotes the $i$-th row of $A_{1}$, and

$$
\Gamma_{\Pi}=\left(\begin{array}{ll}
0 & 0 \\
0 & 0 \\
0 & 0 \\
0 & 0 \\
\left(\theta^{\text {new }}\right)^{-1} E_{\varphi \varphi} & \left(\theta^{\text {new }}\right)^{-1}(1-\delta) E_{\varphi \psi} \\
0 & 0 \\
0 & 0 \\
1 & 0 \\
0 & 1
\end{array}\right)
$$

The solution implies actual surprise coefficients $\left(\widetilde{V}_{1}, \widetilde{V}_{2}\right)$ and we can use a root finding routine to solve for surprise coefficients $A_{1}$ such that point $\left(\widetilde{V}_{1}, \widetilde{V}_{2}\right)=\left(V_{1}, V_{2}\right)$. This can be achieved by varying one of the surprise coefficients and determining the value for the remaining three such that the fixed point property holds. The solution dynamics are then given for all $t \geq 1$ by

$$
x_{t}=A_{V}+B_{V} x_{t-1}+C_{V} e_{t} .
$$

Before we can determine the losses associated with these alternative belief distortions and the associated equilibrium dynamics, we need to determine the initial value $x_{0}$. The time zero 
first-order conditions of the worst-case belief distortion problem (152) can be written as

$$
\begin{aligned}
& \pi_{0}-\varphi_{0}^{\text {new }}+\psi_{-1}^{\text {new }}\left(\frac{\varphi_{-1}}{\psi_{-1}}\right)=0 \\
& \frac{\Lambda_{y}}{\Lambda_{\pi}} y_{0}^{\text {gap }}+\varphi_{0}^{\text {new }} \kappa_{y}=0 \\
& \frac{\Lambda_{q}}{\Lambda_{\pi}}\left(\widehat{q}_{0}^{u}-\widehat{q}_{0}^{u *}\right)+\varphi_{0}^{n e w} \kappa_{q}-\psi_{0}^{\text {new }}+(1-\delta) \psi_{-1}^{\text {new }}=0 \\
& \frac{\varphi_{-1}}{\psi_{-1}} \pi_{0}+(1-\delta)\left(\widehat{q}_{0}^{u}-\widehat{q}_{0}^{u *}\right)-G_{0}=0,
\end{aligned}
$$

where $\frac{\varphi_{-1}}{\psi_{-1}}$ and $G_{0}$ are (fixed) coefficient from the initial pre-commitment, which assume the same value as in the upper bound solution. We can insure that conditions (197)-(200) hold in $t=0$ by choosing appropriate initial conditions for $x_{-1}$ and then letting $x_{0}=A_{V}+B_{V} x_{-1}$, which assumes - as in the upper bound solution - that $e_{0}=0$. Since the columns in $B_{V}$ multiplying $\left(\pi_{-1}, \widehat{q}_{-1}^{u}\right)$ are zero, these values can be chosen arbitrarily. The initial values for the lagged disturbances assume the same value as in the upper bound, i.e., $\widehat{u}_{-1}=\widehat{\bar{\xi}}_{-1}=0$. It thus remains to determine the five remaining initial values $\left(y_{-1}^{g a p}, \zeta_{-1}^{n e w \prime}, E_{-} \pi_{0}, E_{-1} \widehat{q}_{0}^{u}\right)$, where $\zeta_{-1}^{\text {new }}=\left(\varphi_{-1}^{\text {new }},(1-\delta) \psi_{-1}^{\text {new }}\right)$. These are determined by the requirement that (198)-(200) hold, in addition to (197). Equation (197) holds if

$$
\begin{aligned}
\psi_{-1}^{\text {new }}\left(\frac{\varphi_{-1}}{\psi_{-1}}\right)+\frac{\Lambda_{y}}{\Lambda_{\pi} \kappa_{y}} y_{-1}^{g a p} & =0 \\
\frac{\theta^{-1}}{\Lambda_{\pi}} E_{\varphi \varphi}\left(\pi_{0}-E_{-1} \pi_{0}\right)+\frac{\theta^{-1}}{\Lambda_{\pi}}(1-\delta) E_{\varphi \psi}\left(\widehat{q}_{0}^{u}-E_{t} \widehat{q}_{0}^{u}\right) & =0
\end{aligned}
$$

because (197) is then implied by the target criterion (123), which is satisfied for the dynamics determined by $\left(A_{V}, B_{V}, C_{V}\right)$. Equations (198)-(202) determine the five remaining initial conditions $x_{-1}$, such that $x_{0}=A_{V}+B_{V} x_{-1}$ satisfies (197)-(200). It now remains to evaluate the objective function associated with the alternative solution $\left\{x_{t}\right\}_{t=0}^{\infty}$.

The period contributions of some outcome $x_{t}$ to the objective can be written as $\frac{1}{2} \beta^{t} x_{t}^{\prime} \Lambda x_{t}$, where

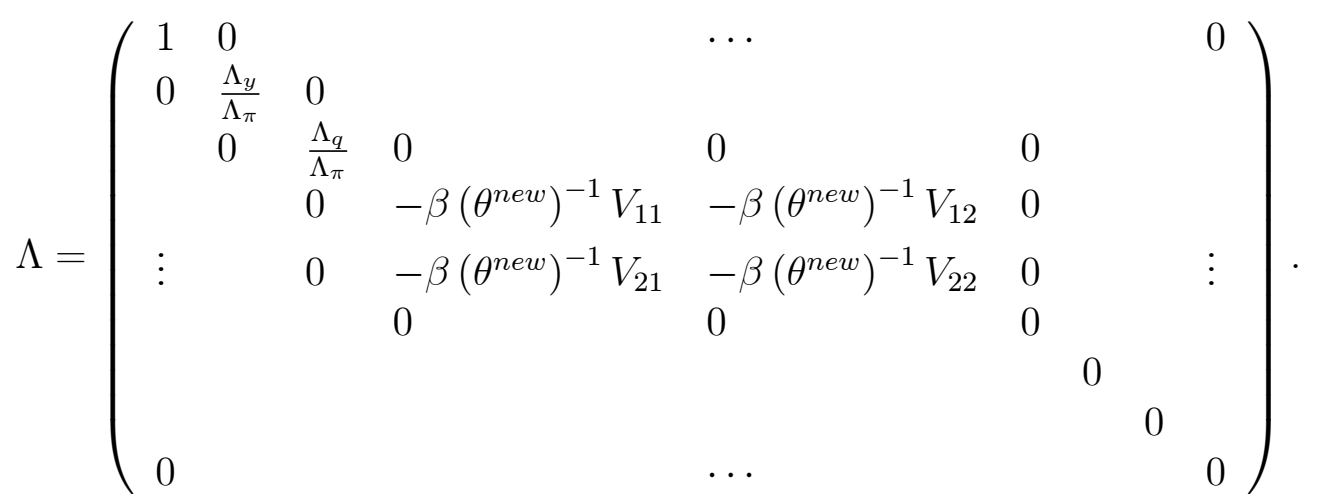


Furthermore, we have that

$$
\begin{aligned}
E_{0}\left[x_{t}^{\prime} \Lambda x_{t}\right] & =E_{0}\left[\left(A_{V}+B_{V} x_{t-1}+C_{V} e_{t}\right)^{\prime} \Lambda\left(A_{V}+B_{V} x_{t-1}+C_{V} e_{t}\right)\right] \\
& =E_{0}\left[\left(A_{V}+B_{V} x_{t-1}\right)^{\prime} \Lambda\left(A_{V}+B_{V} x_{t-1}\right)\right]+\underbrace{E_{0}\left[e_{t}^{\prime} C_{V}^{\prime} \Lambda C_{V} e_{t}\right]}_{=\operatorname{diag}\left(C_{V}^{\prime} \Lambda C_{V}\right)^{\prime} \operatorname{diag}\left(\Sigma_{e}\right)} \\
& =E_{0}\left[\left(A_{V}+B_{V}\left(A_{V}+B_{V} x_{t-2}+C_{V} e_{t-1}\right)\right)^{\prime} \Lambda\left(A_{V}+B_{V}\left(A_{V}+B_{V} x_{t-2}+C_{V} e_{t-1}\right)\right)\right] \\
& +\operatorname{diag}\left(C_{V}^{\prime} \Lambda C_{V}\right)^{\prime} \operatorname{diag}\left(\Sigma_{e}\right) \\
& =E_{0}\left[\left(A_{V}+B_{V}\left(A_{V}+B_{V} x_{t-2}\right)\right)^{\prime} \Lambda\left(A_{V}+B_{V}\left(A_{V}+B_{V} x_{t-2}\right)\right)\right] \\
& \left.+\operatorname{diag}\left(C_{V}^{\prime} \Lambda C_{V}\right)^{\prime} \operatorname{diag}\left(\Sigma_{e}\right)+\operatorname{diag}\left(C_{V}^{\prime} B_{V}^{\prime} \Lambda B_{V} C_{V}\right) \operatorname{diag}\left(\Sigma_{e}\right)\right] \\
& =\ldots \\
& =\left(E_{0} x_{t}\right)^{\prime} \Lambda\left(E_{0} x_{t}\right)+\left(\sum_{j=1}^{t} \operatorname{diag}\left(C_{V}^{\prime} B_{V}^{j-1 \prime} \Lambda B_{V}^{j-1} C_{V}\right)^{\prime}\right) \operatorname{diag}\left(\Sigma_{e}\right)
\end{aligned}
$$

where $E_{0} x_{t}$ evolves recursively as

$$
E_{0} x_{t}=A+B E_{0} x_{-1},
$$

with initial condition $E_{0} x_{0}=x_{0}$. We can then numerically evaluate the infinite sum $\frac{1}{2} E_{0}\left[\sum_{j=0}^{\infty} \beta^{j} x_{j}^{\prime} \Lambda x_{j}\right]$, truncating the summation, as before, when the additional contribution reaches a value below $\left(1-\beta \lambda_{B}\right) \cdot 10^{-9}$, where $\lambda_{B}$ is the largest eigenvalue of $B$.

Figure 1 in the main text depicts the distribution of welfare losses relative to the losses associated with the upper-bound distortions, for 1000 random alternative belief distortion choices in the neighborhood of the upper-bound distortions. We draw 1000 alternative values from a uniform distribution with support $+/-1 \%$ around the upper-bound values for $V$ and from a support of $+/-0.5 \%$ of the upper-bound value of the coefficient $(2,2)$ in the matrix $A_{1}$ in $(192)$ (the remaining coefficients in $A_{1}$ are chosen so as to be consistent with the considered value of $V)$. The figure shows that additional losses are negative, i.e., that alternative belief distortions lead to significantly lower losses for the policymaker than the upper bound distortions. ${ }^{35}$ This shows that that the target criterion (123) implements the upper-bound solution as worst-case outcome.

\section{Impulse Response to Mark-up Shock}

Figure 3 depicts the response to a positive mark-up shock surprise of one standard deviation. Except for the persistent differences in the output gap, housing price and housing price gap, the responses under RE and the upper bound are very similar. Since house prices under RE do not react following the mark-up shock, leaning against the wind is not required under robustly optimal policy in response to mark-up shocks.

\footnotetext{
${ }^{35}$ While 3 of the 1000 considered losses turn out to be positive, the incremental gains are tiny in these cases, when compared to the losses depicted in figure 1 and thus very likely the result of numerical imprecisions: the three positive welfare gains amount to $0.000006189485077,0.000014156768140$ and 0.000017972231905 , respectively.
} 

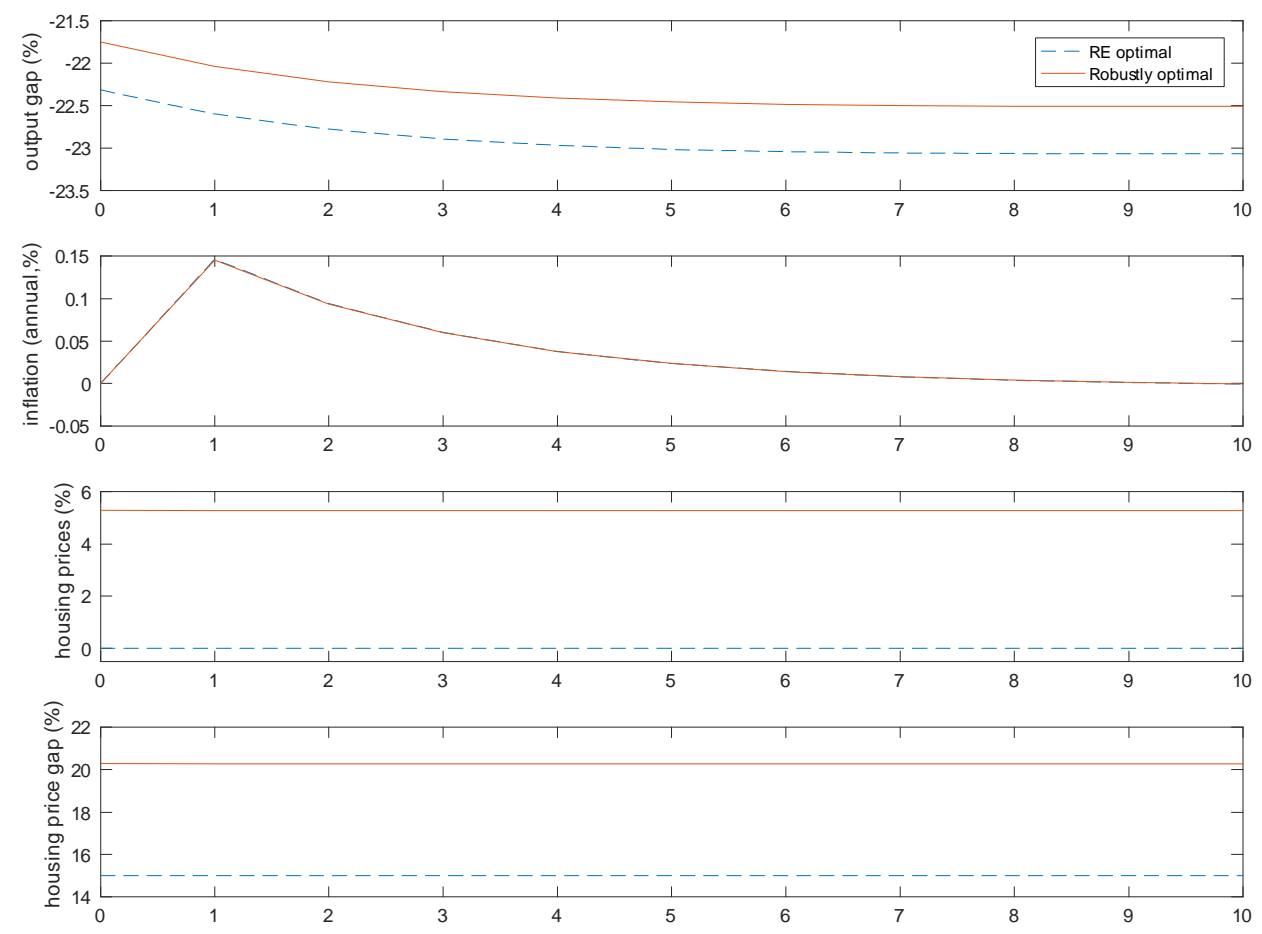

Figure 3: RE vs. robustly optimal dynamics in response to a positive mark-up shock (1 std. deviation) 